Supporting Information

\title{
Charge Transfer and Delocalization in Ladder-Type Fused Bithiophene Imide Oligomer
}

Yujing Jin, Jing-Ai Qiao, Chang Liu, Ling Luo, Xin Chi, Yuexing Zhang*, Ming-Hua Zeng*

Hubei Collaborative Innovation Center for Advanced Organic Chemical Materials, Ministry of Education Key Laboratory for the Synthesis and Application of Organic Functional Molecules, College of Chemistry and Chemical Engineering, Hubei University, Wuhan 430062, P. R. China.

Email: zhangyuexing@sdu.edu.cn (YZ),_zmh@mailbox.gxnu.edu.cn (MZ) 


\section{Contents:}

Figure S1. Top (left) and side (right) views of the optimized geometries of BTI oligomers at LCBLYP*(PCM, solid)/6-31G(d,p) level. The Alkyl side chains are replaced by methyl groups.

Table S1. The effects of different alkyl side chain lengths on the electronic properties (all in $\mathrm{eV}$ ) of BTI2.

Table S2. The bond length of the alternating single and double bonds, average length of the single bonds, average length of the double bonds, bond length alternation (BLA, defined as the average length of single bonds minus the average value of double-bonds for the conjugated $\mathrm{C}-\mathrm{C} / \mathrm{C}=\mathrm{C}$ bond) of neutral molecules of BTI-BTI8 and their cations and anions calculated with LC-BLYP*(PCM, solid), LC-BLYP*, and B3LYP functionals with 6-31G (d,p) basis set. The average absolute bond length change of cations and anion relative to neutral molecules $(<|\mathrm{C}-\mathrm{N}|>$ and $\langle|\mathrm{A}-\mathrm{N}|>)$ are also given. All lengths and length changes in Angstroms.

Figure S2. Atomic labeling in the BTI series of oligomers.

Figure S3. Changes in bond length in the BTI7 and BTI8 oligomers upon oxidation calculated at the LC-BLYP*(PCM)/6-31G(d), LC-BLYP*/6-31G(d) and B3LYP/6-31G(d) levels. Bond indices correspond to the $\mathrm{C}-\mathrm{C}$ single and double bonds starting from one(end) towards the center, see Figure S2.

Figure S4. The evolution of the total bond length of cations/anions relative to neutral states (a), and the evolution of bond length alternation(BLA) for cations/anions/ neutral (b) of BTI-BTI8 calculated at the LC-BLYP*(PCM)/6-31G(d), LC-BLYP*/6-31G(d,p) and B3LYP/6-31G (d,p) levels. Bond lengths in Angstroms.

Table S3. The frontier orbitals energies (HOMO and LUMO), HOMO-LUMO gaps, optical gaps, the vertical ionization potentials $\left(\mathrm{IP}_{\mathrm{v}}\right)$ and electron affinity energies $\left(\mathrm{EA}_{\mathrm{v}}\right)$, hole and electron reorganization energies $\left(\lambda_{+}\right.$and $\left.\lambda_{-}\right)$and hole and electron-transfer mobilities $\left(\mu_{+}\right.$and $\left.\mu_{-}\right)$of BTIBTI8 calculated with LC-BLYP*(PCM, solid), LC-BLYP*(chlorobenzene), LC-BLYP*, and B3LYP methods with 6-31G(d,p) basis set. All energies in $\mathrm{eV}$ and mobilities in $\mathrm{cm}^{2} \mathrm{~V}^{-1} \mathrm{~S}^{-1}$.

Table S4. Calculated electronic structural properties of the BTI analogues at a B3LYP/6-31G(d,p) level.

Figure S5. Simulated maps of the frontier molecular orbitals of BTI3-BTI8 based on the optimized geometries at LC-BLYP*(PCM, solid)/6-31G(d,p) level.

Figure S6. (a)The frontier molecular orbital energy level, (b) the vertical ionization potential and vertical electron affinity, (c) hole and electron reorganization energies, and (d) hole and electrontransfer mobilities changing with reciprocal of the double bond $(1 / \mathrm{n})$ or oligomer length of BTIBTI8 calculated at LC-BLYP*/6-31G(d,p) and B3LYP/6-31G(d,p) levels.

Figure S7. The main charge transport paths in the simulated crystals of BTI (a), BTI2 (b), BTI3 (c) BTI6 (d), BTI7 (e), BTI8 (f).

Figure S8. Frontier molecular orbitals (HOMO and LUMO) in the dimers BTI4-BTI7 with maximum charge transfer integrals.

Table S5. Charge transfer paths, transfer distances, and hole and electron charge transfer integrals in the simulated crystals of BTI series.

Table S6. The evolution of absolute values of transfer integrals $(\mathrm{eV})$ with inter-molecular distances $\left(\mathrm{d}_{\mathrm{P}}\right)$, short $\left(\mathrm{d}_{\mathrm{S}}\right)$ and long $\left(\mathrm{d}_{\mathrm{L}}\right)$ axis displacements by sliding one molecule over the other in BTI3 dimers model calculated at B3LYP/6-31G(d,p) level.

Figure S9. Spin density map in BTI-BTI8 cations calculated at the (a) LC-BLYP*/6-31G(d,p) 
and (b) B3LYP/6-31G(d,p) levels in a vacuum.

Figure S10. Spin density map of the separated polarons of (a) BTI3-BTI8 calculated at the LCBLYP*/6-31G(d,p) and (b) of BTI4-BTI8 calculated at the B3LYP/6-31G(d,p) level in a vacuum.

Figure S11. Spin density map in BTI2-BTI8 bipolarons calculated at the (a) LC-BLYP*/6$31 \mathrm{G}(\mathrm{d}, \mathrm{p})$ and $(\mathrm{b}) \mathrm{B} 3 \mathrm{LYP} / 6-31 \mathrm{G}(\mathrm{d}, \mathrm{p})$ levels in a vacuum.

Table S7. Comparison of the calculated spin density distributions in the BTI-BTI8 cations/ separated polarons /bipolarons calculated at the LC-BLYP*(PCM, solid)/6-31G(d,p) level.

Figure S12. $(a, b)$ Distribution of spin density calculated at the LC-BLYP*/6-31G(d,p) level on each T unit in the BTI-BTI8 cations.(c) in the BTI3-BT8 separated polarons.(d) in the BTI2-BTI8 bipolarons in a vacuum.

Table S8. Comparison of the calculated spin density distributions in the BTI-BTI8 cations/ separated polarons /bipolarons calculated at the LC-BLYP*/6-31G(d,p) level.

Figure S13. (a,b) Distribution of spin density calculated at the B3LYP/6-31G(d,p) level on each $T$ units in the BTI-BTI8 cations.(c) in the BTI4-BT8 separated polarons.(d) in the BTI-BTI8 Bipolarons in a vacuum.

Table S9. Comparison of the calculated spin density distributions in the BTI-BTI5 cations/ separated polarons /bipolarons calculated at the B3LYP/6-31G(d,p) level in a vacuum.

Complete list of authors for Ref. 36 . 
BTI
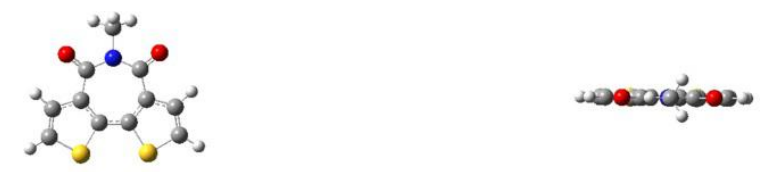

BTI2

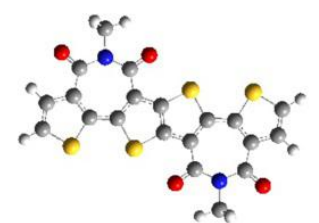

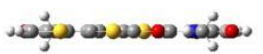

BTI3
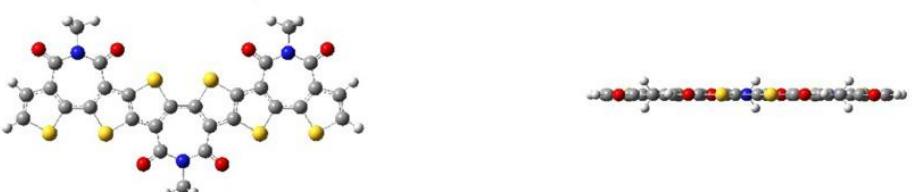

BTI4

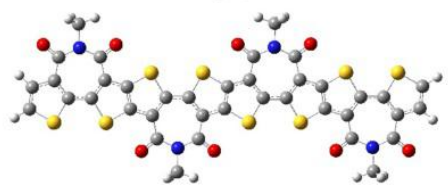

د

BTI5
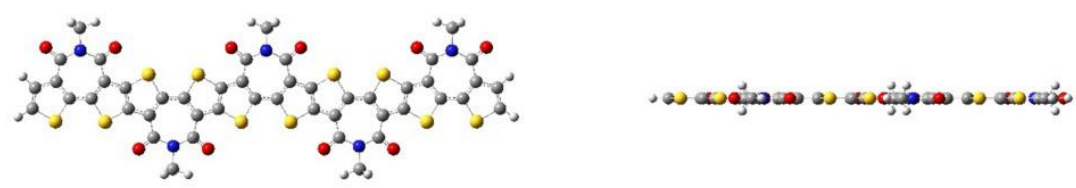

BTI6
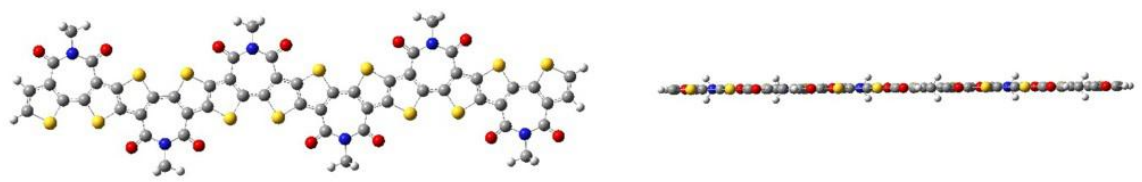

BTI7
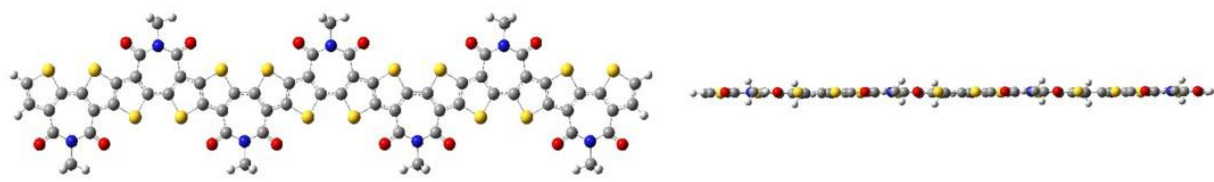

BTI8

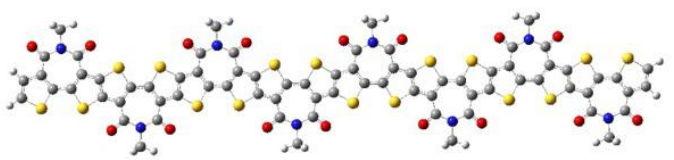

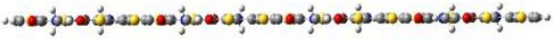

Figure S1. Top (left) and side (right) views of the optimized geometries of BTI oligomers at LCBLYP*(PCM, solid)/6-31G(d,p) level. The Alkyl side chains are replaced by methyl groups. 
Table S1. The effects of different alkyl side chain lengths on the electronic properties (all in $\mathrm{eV}$ ) of BTI2.

\begin{tabular}{|c|c|c|c|c|c|c|c|c|}
\hline Molecules & HOMO & LUMO & IP $_{\text {adia }}$ & EA $_{\text {adia }}$ & IP $_{\text {vert }}$ & EA $_{\text {vert }}$ & $\lambda_{+}$ & $\lambda-$ \\
\hline${\text { BTI2- } \mathrm{CH}_{3}}$ & -5.86 & -2.83 & 7.12 & 6.97 & 1.60 & 1.73 & 0.30 & 0.28 \\
\hline $\mathrm{BTI}_{2}-\mathrm{C}_{2} \mathrm{H}_{5}$ & -5.84 & -2.82 & 7.09 & 6.94 & 1.60 & 1.74 & 0.30 & 0.28 \\
\hline $\mathrm{BTI}_{2}-\mathrm{C}_{3} \mathrm{H}_{7}$ & -5.83 & -2.81 & 7.07 & 6.92 & 1.60 & 1.74 & 0.30 & 0.28 \\
\hline $\mathrm{BTI}_{2}-\mathrm{C}_{4} \mathrm{H}_{9}$ & -5.83 & -2.81 & 7.06 & 6.91 & 1.60 & 1.75 & 0.30 & 0.28 \\
\hline
\end{tabular}

Table S2. The bond length of the alternating single and double bonds, average length of the single bonds, average length of the double bonds, bond length alternation (BLA, defined as the average length of single bonds minus the average value of double-bonds for the conjugated $\mathrm{C}-\mathrm{C} / \mathrm{C}=\mathrm{C}$ bond) of neutral molecules of BTI-BTI8 and their cations and anions calculated with LC-BLYP*(PCM, solid), LC-BLYP*, and B3LYP functionals with $6-31 \mathrm{G}(\mathrm{d}, \mathrm{p})$ basis set. The average absolute bond length change of cations and anion relative to neutral molecules $(<|\mathrm{C}-\mathrm{N}|>$ and $<|\mathrm{A}-\mathrm{N}|>)$ are also given. All lengths and length changes in Angstroms.

\begin{tabular}{|c|c|c|c|c|c|c|c|c|c|c|c|}
\hline Functionals & molecules & $\omega$ & Bonds & $\begin{array}{l}\text { Single } \\
\text { bonds }\end{array}$ & $\begin{array}{l}\text { Bond } \\
\text { length }\end{array}$ & $\begin{array}{c}\text { Double } \\
\text { bonds }\end{array}$ & $\begin{array}{l}\text { Bond } \\
\text { length }\end{array}$ & $\begin{array}{l}\text { Average } \\
\text { single }\end{array}$ & $\begin{array}{l}\text { Average } \\
\text { double }\end{array}$ & BLA & $\begin{array}{l}<|\mathbf{C}-\mathbf{N}|> \\
\langle|\mathbf{A}-\mathbf{N}|>\end{array}$ \\
\hline \multirow{8}{*}{$\begin{array}{l}\text { LC-BLYP* } \\
\text { (PCM)/6-31G } \\
(\mathrm{d}, \mathrm{p})\end{array}$} & \multirow{3}{*}{ BTI } & \multirow{3}{*}{0.066} & Neutral & $\begin{array}{l}\mathrm{C}^{11}-\mathrm{C}^{12} \\
\mathrm{C}^{13}-\mathrm{C}^{17} \\
\mathrm{C}^{18}-\mathrm{C}^{19}\end{array}$ & $\begin{array}{l}1.437 \\
1.431 \\
1.436\end{array}$ & $\begin{array}{l}\mathrm{C}^{10}=\mathrm{C}^{11} \\
\mathrm{C}^{12}=\mathrm{C}^{13} \\
\mathrm{C}^{17}=\mathrm{C}^{18} \\
\mathrm{C}^{19}=\mathrm{C}^{20}\end{array}$ & $\begin{array}{l}1.368 \\
1.399 \\
1.398 \\
1.368\end{array}$ & 1.435 & 1.383 & 0.051 & -- \\
\hline & & & Cation & $\begin{array}{l}\mathrm{C}^{11}-\mathrm{C}^{12} \\
\mathrm{C}^{13}-\mathrm{C}^{17} \\
\mathrm{C}^{18}-\mathrm{C}^{19}\end{array}$ & $\begin{array}{l}1.408 \\
1.399 \\
1.407\end{array}$ & $\begin{array}{l}\mathrm{C}^{10}=\mathrm{C}^{11} \\
\mathrm{C}^{12}=\mathrm{C}^{13} \\
\mathrm{C}^{17}=\mathrm{C}^{18} \\
\mathrm{C}^{19}=\mathrm{C}^{20}\end{array}$ & $\begin{array}{l}1.395 \\
1.425 \\
1.424 \\
1.395\end{array}$ & 1.405 & 1.410 & -0.005 & 0.0143 \\
\hline & & & Anion & $\begin{array}{l}\mathrm{C}^{11}-\mathrm{C}^{12} \\
\mathrm{C}^{13}-\mathrm{C}^{17} \\
\mathrm{C}^{18}-\mathrm{C}^{19}\end{array}$ & $\begin{array}{l}1.436 \\
1.394 \\
1.436\end{array}$ & $\begin{array}{l}\mathrm{C}^{10}=\mathrm{C}^{11} \\
\mathrm{C}^{12}=\mathrm{C}^{13} \\
\mathrm{C}^{17}=\mathrm{C}^{18} \\
\mathrm{C}^{19}=\mathrm{C}^{20}\end{array}$ & $\begin{array}{l}1.367 \\
1.432 \\
1.432 \\
1.367\end{array}$ & 1.422 & 1.399 & 0.022 & 0.0149 \\
\hline & \multirow{3}{*}{ BTI2 } & \multirow{3}{*}{0.038} & Neutral & $\begin{array}{l}\mathrm{C}^{16}-\mathrm{C}^{17} \\
\mathrm{C}^{13}-\mathrm{C}^{15} \\
\mathrm{C}^{11}-\mathrm{C}^{12} \\
\mathrm{C}^{10}-\mathrm{C}^{39} \\
\mathrm{C}^{34}-\mathrm{C}^{38} \\
\mathrm{C}^{32}-\mathrm{C}^{33}\end{array}$ & $\begin{array}{l}1.437 \\
1.427 \\
1.428 \\
1.428 \\
1.427 \\
1.437\end{array}$ & $\begin{array}{l}C^{17}=C^{18} \\
C^{15}=C^{16} \\
C^{12}=C^{13} \\
C^{10}=C^{11} \\
C^{38}=C^{39} \\
C^{33}=C^{34} \\
C^{31}=C^{32}\end{array}$ & $\begin{array}{l}1.370 \\
1.404 \\
1.406 \\
1.392 \\
1.406 \\
1.404 \\
1.370\end{array}$ & 1.431 & 1.393 & 0.037 & -- \\
\hline & & & Cation & $\begin{array}{l}\mathrm{C}^{16}-\mathrm{C}^{17} \\
\mathrm{C}^{13}-\mathrm{C}^{15} \\
\mathrm{C}^{11}-\mathrm{C}^{12} \\
\mathrm{C}^{10}-\mathrm{C}^{39} \\
\mathrm{C}^{34}-\mathrm{C}^{38} \\
\mathrm{C}^{33}-\mathrm{C}^{33}\end{array}$ & $\begin{array}{l}1.420 \\
1.409 \\
1.408 \\
1.408 \\
1.409 \\
1.420\end{array}$ & $\begin{array}{l}C^{17}=C^{18} \\
C^{15}=C^{16} \\
C^{12}=C^{13} \\
C^{10}=C^{11} \\
C^{38}=C^{39} \\
C^{33}=C^{34} \\
C^{31}=C^{32}\end{array}$ & $\begin{array}{l}1.384 \\
1.417 \\
1.421 \\
1.418 \\
1.421 \\
1.417 \\
1.384 \\
\end{array}$ & 1.412 & 1.409 & 0.003 & 0.0090 \\
\hline & & & Anion & $\begin{array}{l}\mathrm{C}^{16}-\mathrm{C}^{17} \\
\mathrm{C}^{13}-\mathrm{C}^{15} \\
\mathrm{C}^{11}-\mathrm{C}^{12} \\
\mathrm{C}^{10}-\mathrm{C}^{39} \\
\mathrm{C}^{34}-\mathrm{C}^{38} \\
\mathrm{C}^{3}-\mathrm{C}^{33}\end{array}$ & $\begin{array}{l}1.440 \\
1.404 \\
1.419 \\
1.419 \\
1.404 \\
1.440\end{array}$ & $\begin{array}{l}C^{17}=C^{18} \\
C^{15}=C^{16} \\
C^{12}=C^{13} \\
C^{10}=C^{11} \\
C^{38}=C^{39} \\
C^{33}=C^{34} \\
C^{31}=C^{32}\end{array}$ & $\begin{array}{l}1.367 \\
1.421 \\
1.427 \\
1.400 \\
1.427 \\
1.421 \\
1.367\end{array}$ & 1.421 & 1.404 & 0.017 & 0.0092 \\
\hline & \multirow[t]{2}{*}{ BTI3 } & \multirow[t]{2}{*}{0.025} & Neutral & $\begin{array}{l}C^{39}-C^{54} \\
C^{51}-C^{53} \\
C^{18}-C^{50} \\
C^{16}-C^{17} \\
C^{13}-C^{15} \\
C^{11}-C^{12} \\
C^{10}-C^{37} \\
C^{32}-C^{36} \\
C^{30}-C^{31}\end{array}$ & $\begin{array}{l}1.437 \\
1.428 \\
1.428 \\
1.427 \\
1.423 \\
1.428 \\
1.428 \\
1.428 \\
1.438\end{array}$ & $\begin{array}{l}\mathrm{C}^{39}=\mathrm{C}^{40} \\
\mathrm{C}^{53}=\mathrm{C}^{54} \\
\mathrm{C}^{50}=\mathrm{C}^{51} \\
\mathrm{C}^{17}=\mathrm{C}^{18} \\
\mathrm{C}^{15}=\mathrm{C}^{16} \\
\mathrm{C}^{12}=\mathrm{C}^{13} \\
\mathrm{C}^{10}=\mathrm{C}^{11} \\
\mathrm{C}^{33}=\mathrm{C}^{37} \\
\mathrm{C}^{31}=\mathrm{C}^{32} \\
\mathrm{C}^{29}=\mathrm{C}^{30}\end{array}$ & $\begin{array}{l}1.371 \\
1.406 \\
1.407 \\
1.394 \\
1.411 \\
1.412 \\
1.394 \\
1.407 \\
1.406 \\
1.371\end{array}$ & 1.429 & 1.398 & 0.031 & -- \\
\hline & & & Cation & $\begin{array}{l}C^{39}-C^{54} \\
C^{51}-C^{53} \\
C^{18}-C^{50} \\
C^{16}-C^{17} \\
C^{13}-C^{15} \\
\end{array}$ & $\begin{array}{l}1.426 \\
1.417 \\
1.415 \\
1.410 \\
1.406 \\
\end{array}$ & $\begin{array}{l}\mathrm{C}^{39}=\mathrm{C}^{40} \\
\mathrm{C}^{53}=\mathrm{C}^{54} \\
\mathrm{C}^{50}=\mathrm{C}^{51} \\
\mathrm{C}^{17}=\mathrm{C}^{18} \\
\mathrm{C}^{15}=\mathrm{C}^{16}\end{array}$ & $\begin{array}{l}1.380 \\
1.414 \\
1.416 \\
1.413 \\
1.423 \\
\end{array}$ & 1.416 & 1.409 & 0.007 & 0.0066 \\
\hline
\end{tabular}




\begin{tabular}{|c|c|c|c|c|c|c|c|c|c|c|c|}
\hline & & & & $\begin{array}{l}C^{11}-C^{12} \\
C^{10}-C^{37} \\
C^{32}-C^{36} \\
C^{30}-C^{31}\end{array}$ & $\begin{array}{l}1.411 \\
1.414 \\
1.416 \\
1.426\end{array}$ & $\begin{array}{l}\mathrm{C}^{12}=\mathrm{C}^{13} \\
\mathrm{C}^{10}=\mathrm{C}^{11} \\
\mathrm{C}^{36}=\mathrm{C}^{37} \\
\mathrm{C}^{31}=\mathrm{C}^{32} \\
\mathrm{C}^{29}=\mathrm{C}^{30}\end{array}$ & $\begin{array}{l}1.424 \\
1.413 \\
1.416 \\
1.414 \\
1.380\end{array}$ & & & & \\
\hline \multirow{6}{*}{$\begin{array}{l}\text { LC-BLYP* } \\
\text { (PCM)/6-31G } \\
\text { (d,p) }\end{array}$} & & & Anion & $\begin{array}{l}C^{39}-C^{54} \\
C^{51}-C^{53} \\
C^{18}-C^{50} \\
C^{16}-C^{17} \\
C^{13}-C^{15} \\
C^{11}-C^{12} \\
C^{10}-C^{37} \\
C^{32}-C^{36} \\
C^{30}-C^{31}\end{array}$ & $\begin{array}{l}1.441 \\
1.414 \\
1.421 \\
1.421 \\
1.400 \\
1.422 \\
1.421 \\
1.414 \\
1.441\end{array}$ & $\begin{array}{l}\mathrm{C}^{39}=\mathrm{C}^{40} \\
\mathrm{C}^{53}=\mathrm{C}^{54} \\
\mathrm{C}^{50}=\mathrm{C}^{51} \\
\mathrm{C}^{17}=\mathrm{C}^{18} \\
\mathrm{C}^{15}=\mathrm{C}^{16} \\
\mathrm{C}^{12}=\mathrm{C}^{13} \\
\mathrm{C}^{10}=\mathrm{C}^{11} \\
\mathrm{C}^{36}=\mathrm{C}^{37} \\
\mathrm{C}^{31}=\mathrm{C}^{32} \\
\mathrm{C}^{29}=\mathrm{C}^{30}\end{array}$ & $\begin{array}{l}1.368 \\
1.416 \\
1.419 \\
1.400 \\
1.429 \\
1.431 \\
1.399 \\
1.419 \\
1.415 \\
1.368\end{array}$ & 1.422 & 1.406 & 0.015 & 0.0068 \\
\hline & \multirow{3}{*}{ BTI4 } & \multirow{3}{*}{0.018} & Neutral & $\begin{array}{l}C^{56}-C^{71} \\
C^{68}-C^{70} \\
C^{37}-C^{67} \\
C^{35}-C^{36} \\
C^{32}-C^{34} \\
C^{30}-C^{31} \\
C^{29}-C^{54} \\
C^{51}-C^{53} \\
C^{49}-C^{50} \\
C^{18}-C^{48} \\
C^{13}-C^{17} \\
C^{11}-C^{12}\end{array}$ & $\begin{array}{l}1.438 \\
1.428 \\
1.430 \\
1.427 \\
1.423 \\
1.428 \\
1.428 \\
1.423 \\
1.427 \\
1.430 \\
1.428 \\
1.438\end{array}$ & $\begin{array}{l}C^{56}=C^{57} \\
C^{70}=C^{71} \\
C^{67}=C^{68} \\
C^{36}=C^{37} \\
C^{34}=C^{35} \\
C^{31}=C^{32} \\
C^{29}=C^{30} \\
C^{50}=C^{51} \\
C^{53}=C^{54} \\
C^{48}=C^{49} \\
C^{17}=C^{18} \\
C^{12}=C^{13} \\
C^{10}=C^{11}\end{array}$ & $\begin{array}{l}1.371 \\
1.406 \\
1.408 \\
1.395 \\
1.412 \\
1.413 \\
1.396 \\
1.412 \\
1.413 \\
1.395 \\
1.408 \\
1.406 \\
1.371\end{array}$ & 1.429 & 1.400 & 0.028 & -- \\
\hline & & & Cation & $\begin{array}{l}C^{56}-C^{71} \\
C^{68}-C^{70} \\
C^{37}-C^{67} \\
C^{35}-C^{36} \\
C^{32}-C^{34} \\
C^{30}-C^{31} \\
C^{29}-C^{54} \\
C^{51}-C^{53} \\
C^{49}-C^{50} \\
C^{18}-C^{48} \\
C^{13}-C^{17} \\
C^{11}-C^{12}\end{array}$ & $\begin{array}{l}1.430 \\
1.420 \\
1.420 \\
1.414 \\
1.410 \\
1.415 \\
1.415 \\
1.410 \\
1.414 \\
1.420 \\
1.420 \\
1.430\end{array}$ & $\begin{array}{l}C^{56}=C^{57} \\
C^{70}=C^{71} \\
C^{67}=C^{68} \\
C^{36}=C^{37} \\
C^{34}=C^{35} \\
C^{31}=C^{32} \\
C^{29}=C^{30} \\
C^{50}=C^{51} \\
C^{53}=C^{54} \\
C^{48}=C^{49} \\
C^{17}=C^{18} \\
C^{12}=C^{13} \\
C^{10}=C^{11}\end{array}$ & $\begin{array}{l}1.378 \\
1.411 \\
1.414 \\
1.409 \\
1.421 \\
1.422 \\
1.412 \\
1.421 \\
1.422 \\
1.409 \\
1.414 \\
1.411 \\
1.378\end{array}$ & 1.418 & 1.409 & 0.009 & 0.0052 \\
\hline & & & Anion & $\begin{array}{l}C^{56}-C^{71} \\
C^{68}-C^{70} \\
C^{37}-C^{67} \\
C^{35}-C^{36} \\
C^{32}-C^{34} \\
C^{30}-C^{31} \\
C^{29}-C^{54} \\
C^{51}-C^{53} \\
C^{49}-C^{50} \\
C^{18}-C^{48} \\
C^{13}-C^{17} \\
C^{11}-C^{12}\end{array}$ & $\begin{array}{l}1.441 \\
1.419 \\
1.425 \\
1.424 \\
1.405 \\
1.421 \\
1.421 \\
1.405 \\
1.424 \\
1.425 \\
1.419 \\
1.441\end{array}$ & $\begin{array}{l}C^{56}=C^{57} \\
C^{70}=C^{71} \\
C^{67}=C^{68} \\
C^{36}=C^{37} \\
C^{34}=C^{35} \\
C^{31}=C^{32} \\
C^{29}=C^{30} \\
C^{50}=C^{51} \\
C^{53}=C^{54} \\
C^{48}=C^{49} \\
C^{17}=C^{18} \\
C^{12}=C^{13} \\
C^{10}=C^{11}\end{array}$ & $\begin{array}{l}1.369 \\
1.412 \\
1.416 \\
1.398 \\
1.425 \\
1.427 \\
1.402 \\
1.425 \\
1.427 \\
1.398 \\
1.416 \\
1.412 \\
1.369 \\
\end{array}$ & 1.422 & 1.407 & 0.015 & 0.0052 \\
\hline & \multirow[t]{2}{*}{ BTI5 } & \multirow[t]{2}{*}{0.013} & Neutral & $\begin{array}{l}C^{11}-C^{12} \\
C^{13}-C^{17} \\
C^{18}-C^{48} \\
C^{49}-C^{50} \\
C^{51}-C^{53} \\
C^{29}-C^{54} \\
C^{30}-C^{31} \\
C^{32}-C^{34} \\
C^{35}-C^{36} \\
C^{37}-C^{67} \\
C^{68}-C^{70} \\
C^{56}-C^{71} \\
C^{57}-C^{84} \\
C^{85}-C^{87} \\
C^{73}-C^{88}\end{array}$ & $\begin{array}{l}1.439 \\
1.429 \\
1.429 \\
1.428 \\
1.423 \\
1.428 \\
1.428 \\
1.423 \\
1.427 \\
1.428 \\
1.423 \\
1.428 \\
1.429 \\
1.429 \\
1.439\end{array}$ & $\begin{array}{l}C^{73}=C^{74} \\
C^{87}=C^{88} \\
C^{84}=C^{85} \\
C^{56}=C^{57} \\
C^{70}=C^{71} \\
C^{67}=C^{68} \\
C^{36}=C^{37} \\
C^{34}=C^{35} \\
C^{31}=C^{32} \\
C^{29}=C^{30} \\
C^{53}=C^{54} \\
C^{50}=C^{51} \\
C^{48}=C^{49} \\
C^{17}=C^{18} \\
C^{12}=C^{13} \\
C^{10}=C^{11}\end{array}$ & $\begin{array}{l}1.372 \\
1.407 \\
1.408 \\
1.395 \\
1.412 \\
1.413 \\
1.397 \\
1.412 \\
1.413 \\
1.397 \\
1.413 \\
1.412 \\
1.395 \\
1.408 \\
1.407 \\
1.372 \\
\end{array}$ & 1.429 & 1.402 & 0.026 & -- \\
\hline & & & Cation & $\begin{array}{l}C^{11}-C^{12} \\
C^{13}-C^{17} \\
C^{18}-C^{48}\end{array}$ & $\begin{array}{l}1.432 \\
1.423 \\
1.422 \\
\end{array}$ & $\begin{array}{l}C^{73}=C^{74} \\
C^{87}=C^{88} \\
C^{84}=C^{85}\end{array}$ & $\begin{array}{l}1.376 \\
1.411 \\
1.413 \\
\end{array}$ & 1.420 & 1.410 & 0.010 & 0.0045 \\
\hline
\end{tabular}




\begin{tabular}{|c|c|c|c|c|c|c|c|c|c|c|c|}
\hline & & & & $\begin{array}{l}C^{49}-C^{50} \\
C^{51}-C^{53} \\
C^{29}-C^{54} \\
C^{30}-C^{31} \\
C^{32}-C^{34} \\
C^{35}-C^{36} \\
C^{37}-C^{67} \\
C^{68}-C^{70} \\
C^{56}-C^{71} \\
C^{57}-C^{84} \\
C^{85}-C^{87} \\
C^{73}-C^{88}\end{array}$ & $\begin{array}{l}1.417 \\
1.413 \\
1.418 \\
1.416 \\
1.411 \\
1.416 \\
1.418 \\
1.413 \\
1.417 \\
1.422 \\
1.423 \\
1.432\end{array}$ & $\begin{array}{l}C^{56}=C^{57} \\
C^{70}=C^{71} \\
C^{67}=C^{68} \\
C^{36}=C^{37} \\
C^{34}=C^{35} \\
C^{31}=C^{32} \\
C^{29}=C^{30} \\
C^{53}=C^{54} \\
C^{50}=C^{51} \\
C^{48}=C^{49} \\
C^{17}=C^{18} \\
C^{12}=C^{13} \\
C^{10}=C^{11}\end{array}$ & $\begin{array}{l}1.406 \\
1.419 \\
1.420 \\
1.410 \\
1.421 \\
1.421 \\
1.410 \\
1.420 \\
1.419 \\
1.406 \\
1.413 \\
1.411 \\
1.376\end{array}$ & & & & \\
\hline \multirow{4}{*}{$\begin{array}{c}\text { LC-BLYP* } \\
\text { (PCM)/6-31G } \\
(\mathrm{d}, \mathrm{p})\end{array}$} & & & Anion & $\begin{array}{l}C^{11}-C^{12} \\
C^{13}-C^{17} \\
C^{18}-C^{48} \\
C^{49}-C^{50} \\
C^{51}-C^{53} \\
C^{29}-C^{54} \\
C^{30}-C^{31} \\
C^{32}-C^{34} \\
C^{35}-C^{36} \\
C^{37}-C^{67} \\
C^{68}-C^{70} \\
C^{56}-C^{71} \\
C^{57}-C^{84} \\
C^{85}-C^{87} \\
C^{73}-C^{88}\end{array}$ & $\begin{array}{l}1.441 \\
1.423 \\
1.426 \\
1.427 \\
1.409 \\
1.421 \\
1.422 \\
1.406 \\
1.422 \\
1.421 \\
1.409 \\
1.426 \\
1.426 \\
1.423 \\
1.441\end{array}$ & $\begin{array}{l}C^{73}=C^{74} \\
C^{87}=C^{88} \\
C^{84}=C^{85} \\
C^{56}=C^{57} \\
C^{70}=C^{71} \\
C^{67}=C^{68} \\
C^{36}=C^{37} \\
C^{34}=C^{35} \\
C^{31}=C^{32} \\
C^{29}=C^{30} \\
C^{53}=C^{54} \\
C^{50}=C^{51} \\
C^{48}=C^{49} \\
C^{17}=C^{18} \\
C^{12}=C^{13} \\
C^{10}=C^{11}\end{array}$ & $\begin{array}{l}1.370 \\
1.411 \\
1.413 \\
1.397 \\
1.423 \\
1.424 \\
1.401 \\
1.426 \\
1.427 \\
1.401 \\
1.424 \\
1.423 \\
1.397 \\
1.413 \\
1.411 \\
1.370\end{array}$ & 1.423 & 1.4084 & 0.015 & 0.0045 \\
\hline & \multirow{3}{*}{ BTI6 } & \multirow{3}{*}{0.010} & Neutral & $\begin{array}{l}C^{11}-C^{12} \\
C^{13}-C^{17} \\
C^{18}-C^{48} \\
C^{49}-C^{50} \\
C^{51}-C^{53} \\
C^{29}-C^{54} \\
C^{30}-C^{31} \\
C^{32}-C^{34} \\
C^{35}-C^{36} \\
C^{37}-C^{67} \\
C^{68}-C^{70} \\
C^{56}-C^{71} \\
C^{57}-C^{82} \\
C^{83}-C^{85} \\
C^{86}-C^{97} \\
C^{98}-C^{108} \\
C^{99}-C^{101} \\
C^{102}-C^{103}\end{array}$ & $\begin{array}{l}1.439 \\
1.429 \\
1.430 \\
1.428 \\
1.423 \\
1.429 \\
1.428 \\
1.423 \\
1.428 \\
1.428 \\
1.423 \\
1.427 \\
1.429 \\
1.423 \\
1.428 \\
1.430 \\
1.429 \\
1.439\end{array}$ & $\begin{array}{l}\mathrm{C}^{10}=\mathrm{C}^{11} \\
\mathrm{C}^{12}=\mathrm{C}^{13} \\
\mathrm{C}^{17}=\mathrm{C}^{18} \\
\mathrm{C}^{48}=\mathrm{C}^{49} \\
\mathrm{C}^{50}=\mathrm{C}^{51} \\
\mathrm{C}^{53}=\mathrm{C}^{54} \\
\mathrm{C}^{29}=\mathrm{C}^{30} \\
\mathrm{C}^{31}=\mathrm{C}^{32} \\
\mathrm{C}^{34}=\mathrm{C}^{35} \\
\mathrm{C}^{36}=\mathrm{C}^{37} \\
\mathrm{C}^{67}=\mathrm{C}^{68} \\
\mathrm{C}^{70}=\mathrm{C}^{71} \\
\mathrm{C}^{56}=\mathrm{C}^{57} \\
\mathrm{C}^{82}=\mathrm{C}^{83} \\
\mathrm{C}^{85}=\mathrm{C}^{86} \\
\mathrm{C}^{97}=\mathrm{C}^{100} \\
\mathrm{C}^{98}=\mathrm{C}^{99} \\
\mathrm{C}^{101}=\mathrm{C}^{102} \\
\mathrm{C}^{103}=\mathrm{C}^{104}\end{array}$ & $\begin{array}{l}1.372 \\
1.408 \\
1.409 \\
1.396 \\
1.412 \\
1.410 \\
1.397 \\
1.413 \\
1.413 \\
1.397 \\
1.414 \\
1.412 \\
1.397 \\
1.414 \\
1.412 \\
1.396 \\
1.409 \\
1.408 \\
1.372\end{array}$ & 1.428 & 1.403 & 0.025 & -- \\
\hline & & & Cation & $\begin{array}{l}C^{11}-C^{12} \\
C^{13}-C^{17} \\
C^{18}-C^{48} \\
C^{49}-C^{50} \\
C^{51}-C^{53} \\
C^{29}-C^{54} \\
C^{30}-C^{31} \\
C^{32}-C^{34} \\
C^{35}-C^{36} \\
C^{37}-C^{67} \\
C^{68}-C^{70} \\
C^{56}-C^{71} \\
C^{57}-C^{82} \\
C^{83}-C^{85} \\
C^{86}-C^{97} \\
C^{98}-C^{108} \\
C^{99}-C^{101} \\
C^{102}-C^{103}\end{array}$ & \begin{tabular}{l|l}
1.434 \\
1.425 \\
1.424 \\
1.420 \\
1.415 \\
1.420 \\
1.418 \\
1.413 \\
1.418 \\
1.418 \\
1.413 \\
1.417 \\
1.420 \\
1.415 \\
1.420 \\
1.424 \\
1.425 \\
1.434
\end{tabular} & $\begin{array}{l}C^{10}=C^{11} \\
C^{12}=C^{13} \\
C^{17}=C^{18} \\
C^{48}=C^{49} \\
C^{50}=C^{51} \\
C^{53}=C^{54} \\
C^{29}=C^{30} \\
C^{31}=C^{32} \\
C^{34}=C^{35} \\
C^{36}=C^{37} \\
C^{67}=C^{68} \\
C^{70}=C^{71} \\
C^{56}=C^{57} \\
C^{82}=C^{83} \\
C^{85}=C^{86} \\
C^{97}=C^{100} \\
C^{98}=C^{99} \\
C^{101}=C^{102} \\
C^{103}=C^{104}\end{array}$ & $\begin{array}{l}1.376 \\
1.410 \\
1.412 \\
1.404 \\
1.418 \\
1.419 \\
1.408 \\
1.420 \\
1.420 \\
1.409 \\
1.421 \\
1.419 \\
1.408 \\
1.419 \\
1.418 \\
1.404 \\
1.412 \\
1.410 \\
1.376\end{array}$ & 1.421 & 1.410 & 0.011 & 0.0037 \\
\hline & & & Anion & $\begin{array}{l}C^{11}-C^{12} \\
C^{13}-C^{17} \\
C^{18}-C^{48} \\
C^{49}-C^{50} \\
C^{51}-C^{53} \\
C^{29}-C^{54} \\
C^{30}-C^{31} \\
C^{32}-C^{34}\end{array}$ & $\begin{array}{l}1.441 \\
1.425 \\
1.428 \\
1.428 \\
1.413 \\
1.423 \\
1.423 \\
1.408\end{array}$ & $\begin{array}{l}\mathrm{C}^{10}=\mathrm{C}^{11} \\
\mathrm{C}^{12}=\mathrm{C}^{13} \\
\mathrm{C}^{17}=\mathrm{C}^{18} \\
\mathrm{C}^{48}=\mathrm{C}^{49} \\
\mathrm{C}^{50}=\mathrm{C}^{51} \\
\mathrm{C}^{53}=\mathrm{C}^{54} \\
\mathrm{C}^{29}=\mathrm{C}^{30} \\
\mathrm{C}^{31}=\mathrm{C}^{32}\end{array}$ & $\begin{array}{l}1.371 \\
1.410 \\
1.412 \\
1.396 \\
1.420 \\
1.421 \\
1.400 \\
1.424\end{array}$ & 1.423 & 1.408 & 0.015 & 0.0039 \\
\hline
\end{tabular}




\begin{tabular}{|c|c|c|c|c|c|c|c|c|c|c|c|}
\hline & & & & $\begin{array}{l}C^{35}-C^{36} \\
C^{37}-C^{67} \\
C^{68}-C^{70} \\
C^{56}-C^{71} \\
C^{57}-C^{82} \\
C^{83}-C^{85} \\
C^{86}-C^{97} \\
C^{98}-C^{108} \\
C^{99}-C^{101} \\
C^{102}-C^{103}\end{array}$ & $\begin{array}{l}1.422 \\
1.422 \\
1.408 \\
1.423 \\
1.423 \\
1.413 \\
1.428 \\
1.428 \\
1.425 \\
1.441\end{array}$ & $\begin{array}{l}C^{34}=C^{35} \\
C^{36}=C^{37} \\
C^{67}=C^{68} \\
C^{70}=C^{71} \\
C^{56}=C^{57} \\
C^{82}=C^{83} \\
C^{85}=C^{86} \\
C^{97}=C^{106} \\
C^{98}=C^{99} \\
C^{101}=C^{102} \\
C^{103}=C^{104}\end{array}$ & $\begin{array}{l}1.425 \\
1.402 \\
1.425 \\
1.424 \\
1.400 \\
1.421 \\
1.420 \\
1.396 \\
1.412 \\
1.410 \\
1.371\end{array}$ & & & & \\
\hline \multirow{3}{*}{$\begin{array}{l}\text { LC-BLYP* } \\
\text { (PCM)/6-31G } \\
\text { (d,p) }\end{array}$} & \multirow{3}{*}{ BTI7 } & \multirow{3}{*}{0.008} & Neutral & $\begin{array}{l}C^{11}-C^{12} \\
C^{13}-C^{17} \\
C^{18}-C^{48} \\
C^{49}-C^{50} \\
C^{51}-C^{53} \\
C^{29}-C^{54} \\
C^{30}-C^{31} \\
C^{32}-C^{34} \\
C^{35}-C^{36} \\
C^{37}-C^{67} \\
C^{68}-C^{70} \\
C^{56}-C^{71} \\
C^{57}-C^{82} \\
C^{83}-C^{85} \\
C^{86}-C^{97} \\
C^{98}-C^{106} \\
C^{99}-C^{101} \\
C^{102}-C^{103} \\
C^{104}-C^{124} \\
C^{119}-C^{123} \\
C^{117}-C^{118}\end{array}$ & $\begin{array}{l}1.439 \\
1.429 \\
1.430 \\
1.428 \\
1.424 \\
1.429 \\
1.428 \\
1.423 \\
1.428 \\
1.428 \\
1.423 \\
1.428 \\
1.428 \\
1.423 \\
1.428 \\
1.429 \\
1.424 \\
1.428 \\
1.430 \\
1.429 \\
1.439\end{array}$ & $\begin{array}{l}C^{10}=C^{11} \\
C^{12}=C^{13} \\
C^{17}=C^{18} \\
C^{48}=C^{49} \\
C^{50}=C^{51} \\
C^{53}=C^{54} \\
C^{29}=C^{30} \\
C^{31}=C^{32} \\
C^{34}=C^{35} \\
C^{36}=C^{37} \\
C^{67}=C^{68} \\
C^{70}=C^{71} \\
C^{56}=C^{57} \\
C^{82}=C^{83} \\
C^{85}=C^{86} \\
C^{97}=C^{106} \\
C^{98}=C^{99} \\
C^{101}=C^{102} \\
C^{103}=C^{104} \\
C^{123}-C^{124} \\
C^{118}-C^{119} \\
C^{116}-C^{117}\end{array}$ & \begin{tabular}{l|l}
1.372 \\
1.408 \\
1.409 \\
1.396 \\
1.412 \\
1.414 \\
1.397 \\
1.413 \\
1.414 \\
1.397 \\
1.414 \\
1.414 \\
1.397 \\
1.414 \\
1.413 \\
1.397 \\
1.414 \\
1.412 \\
1.396 \\
1.409 \\
1.408 \\
1.372
\end{tabular} & 1.428 & 1.404 & 0.024 & -- \\
\hline & & & Cation & $\begin{array}{l}C^{11}-C^{12} \\
C^{13}-C^{17} \\
C^{18}-C^{48} \\
C^{49}-C^{50} \\
C^{51}-C^{53} \\
C^{29}-C^{54} \\
C^{30}-C^{31} \\
C^{32}-C^{34} \\
C^{35}-C^{36} \\
C^{37}-C^{67} \\
C^{68}-C^{70} \\
C^{56}-C^{71} \\
C^{57}-C^{82} \\
C^{83}-C^{85} \\
C^{86}-C^{97} \\
C^{98}-C^{106} \\
C^{99}-C^{101} \\
C^{102}-C^{103} \\
C^{104}-C^{124} \\
C^{119}-C^{123} \\
C^{117}-C^{118}\end{array}$ & $\begin{array}{l}1.435 \\
1.426 \\
1.425 \\
1.422 \\
1.417 \\
1.422 \\
1.419 \\
1.415 \\
1.419 \\
1.419 \\
1.414 \\
1.419 \\
1.419 \\
1.415 \\
1.419 \\
1.422 \\
1.417 \\
1.422 \\
1.425 \\
1.426 \\
1.435\end{array}$ & $\begin{array}{l}C^{10}=C^{11} \\
C^{12}=C^{13} \\
C^{17}=C^{18} \\
C^{48}=C^{49} \\
C^{50}=C^{51} \\
C^{53}=C^{54} \\
C^{29}=C^{30} \\
C^{31}=C^{32} \\
C^{34}=C^{35} \\
C^{36}=C^{37} \\
C^{67}=C^{68} \\
C^{70}=C^{71} \\
C^{56}=C^{57} \\
C^{82}=C^{83} \\
C^{85}=C^{86} \\
C^{97}=C^{106} \\
C^{98}=C^{99} \\
C^{101}=C^{102} \\
C^{103}=C^{104} \\
C^{123}-C^{124} \\
C^{118}-C^{119} \\
C^{116}-C^{117}\end{array}$ & $\begin{array}{l}1.375 \\
1.410 \\
1.411 \\
1.403 \\
1.417 \\
1.418 \\
1.406 \\
1.419 \\
1.419 \\
1.408 \\
1.420 \\
1.420 \\
1.408 \\
1.419 \\
1.419 \\
1.406 \\
1.418 \\
1.417 \\
1.403 \\
1.411 \\
1.410 \\
1.375\end{array}$ & 1.422 & 1.410 & 0.012 & 0.0032 \\
\hline & & & Anion & $\begin{array}{l}C^{11}-C^{12} \\
C^{13}-C^{17} \\
C^{18}-C^{48} \\
C^{49}-C^{50} \\
C^{51}-C^{53} \\
C^{29}-C^{54} \\
C^{30}-C^{31} \\
C^{32}-C^{34} \\
C^{35}-C^{36} \\
C^{37}-C^{67} \\
C^{68}-C^{70} \\
C^{56}-C^{71} \\
C^{57}-C^{82} \\
C^{83}-C^{85} \\
C^{86}-C^{97} \\
C^{98}-C^{106} \\
C^{99}-C^{101} \\
C^{102}-C^{103} \\
C^{104}-C^{124} \\
C^{119}-C^{123}\end{array}$ & $\begin{array}{l}1.441 \\
1.427 \\
1.429 \\
1.428 \\
1.416 \\
1.424 \\
1.425 \\
1.411 \\
1.423 \\
1.423 \\
1.409 \\
1.423 \\
1.423 \\
1.411 \\
1.425 \\
1.424 \\
1.416 \\
1.428 \\
1.429 \\
1.427\end{array}$ & $\begin{array}{l}C^{10}=C^{11} \\
C^{12}=C^{13} \\
C^{17}=C^{18} \\
C^{48}=C^{49} \\
C^{50}=C^{51} \\
C^{53}=C^{54} \\
C^{29}=C^{30} \\
C^{31}=C^{32} \\
C^{34}=C^{35} \\
C^{36}=C^{37} \\
C^{67}=C^{68} \\
C^{70}=C^{71} \\
C^{56}=C^{57} \\
C^{82}=C^{83} \\
C^{85}=C^{86} \\
C^{97}=C^{106} \\
C^{98}=C^{99} \\
C^{101}=C^{102} \\
C^{103}=C^{104} \\
C^{118}-C^{119}\end{array}$ & \begin{tabular}{l|l}
1.371 \\
1.409 \\
1.411 \\
1.396 \\
1.419 \\
1.419 \\
1.400 \\
1.422 \\
1.423 \\
1.401 \\
1.424 \\
1.424 \\
1.401 \\
1.423 \\
1.422 \\
1.400 \\
1.419 \\
1.419 \\
1.396 \\
1.409
\end{tabular} & 1.424 & 1.409 & 0.015 & 0.0033 \\
\hline
\end{tabular}




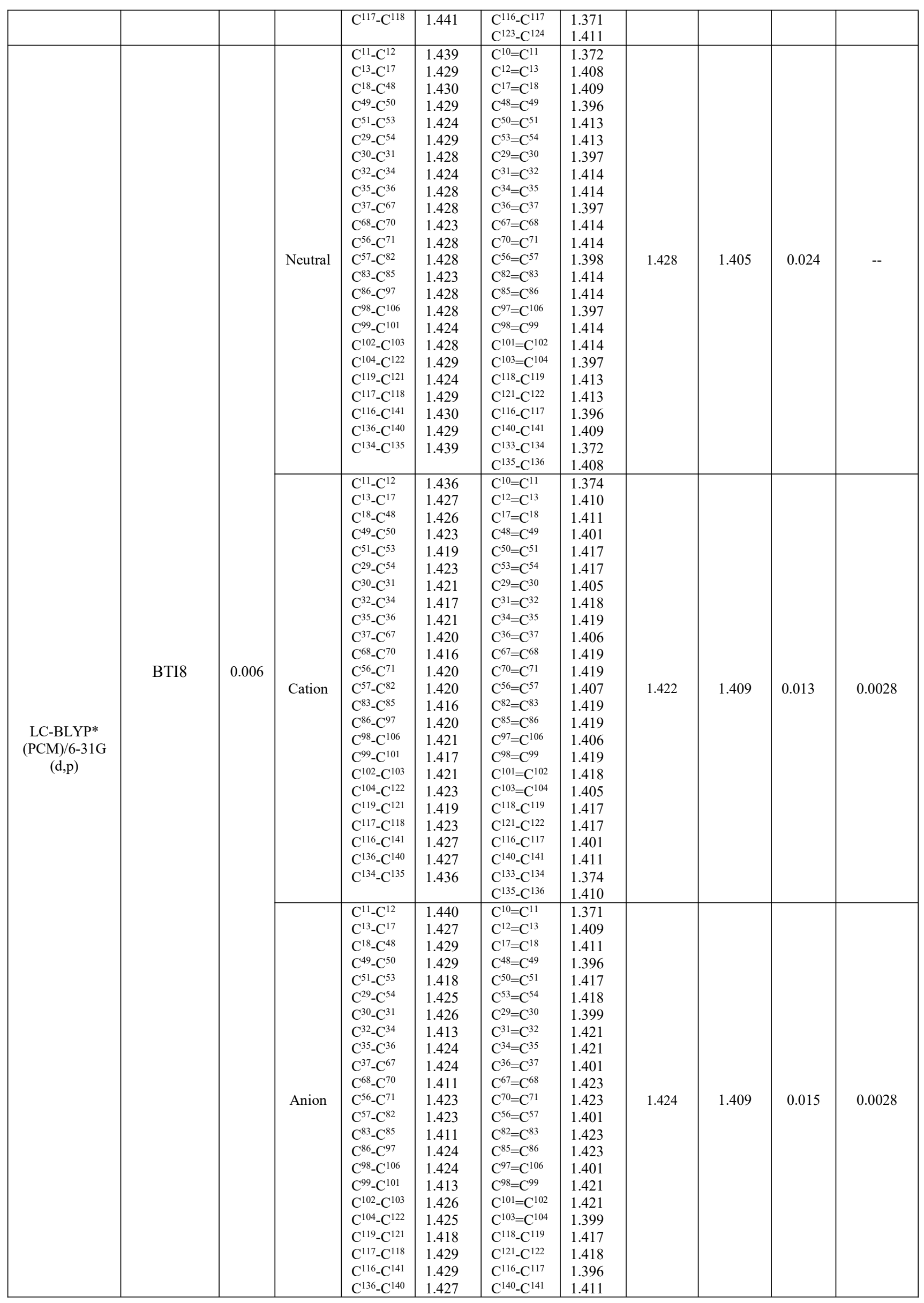




\begin{tabular}{|c|c|c|c|c|c|c|c|c|c|c|c|}
\hline & & & & $\mathrm{C}^{134}-\mathrm{C}^{135}$ & 1.440 & $\begin{array}{l}C^{133}-C^{134} \\
C^{135}-C^{136}\end{array}$ & $\begin{array}{l}1.371 \\
1.409 \\
\end{array}$ & & & & \\
\hline \multirow{10}{*}{$\begin{array}{l}\text { LC-BLYP*/ } \\
6-31 \mathrm{G}(\mathrm{d}, \mathrm{p})\end{array}$} & \multirow{3}{*}{ BTI } & \multirow{3}{*}{$\begin{array}{l}0.235 \\
4\end{array}$} & Neutral & $\begin{array}{l}C^{11}-C^{12} \\
C^{13}-C^{17} \\
C^{18}-C^{19}\end{array}$ & $\begin{array}{l}1.432 \\
1.439 \\
1.431 \\
\end{array}$ & $\begin{array}{l}\mathrm{C}^{10}=\mathrm{C}^{11} \\
\mathrm{C}^{12}=\mathrm{C}^{13} \\
\mathrm{C}^{17}=\mathrm{C}^{18} \\
\mathrm{C}^{19}=\mathrm{C}^{20}\end{array}$ & $\begin{array}{l}1.359 \\
1.382 \\
1.381 \\
1.360\end{array}$ & 1.434 & 1.371 & 0.064 & -- \\
\hline & & & Cation & $\begin{array}{l}C^{11}-C^{12} \\
C^{13}-C^{17} \\
C^{18}-C^{19}\end{array}$ & $\begin{array}{l}1.397 \\
1.393 \\
1.397\end{array}$ & $\begin{array}{l}\mathrm{C}^{10}=\mathrm{C}^{11} \\
\mathrm{C}^{12}=\mathrm{C}^{13} \\
\mathrm{C}^{17}=\mathrm{C}^{18} \\
\mathrm{C}^{19}=\mathrm{C}^{20}\end{array}$ & $\begin{array}{l}1.389 \\
1.419 \\
1.418 \\
1.389 \\
\end{array}$ & 1.396 & 1.403 & -0.008 & 0.0169 \\
\hline & & & Anion & $\begin{array}{l}C^{11}-C^{12} \\
C^{13}-C^{17} \\
C^{18}-C^{19}\end{array}$ & $\begin{array}{l}1.431 \\
1.388 \\
1.430\end{array}$ & $\begin{array}{l}\mathrm{C}^{10}=\mathrm{C}^{11} \\
\mathrm{C}^{12}=\mathrm{C}^{13} \\
\mathrm{C}^{17}=\mathrm{C}^{18} \\
\mathrm{C}^{19}=\mathrm{C}^{20}\end{array}$ & $\begin{array}{l}1.358 \\
1.422 \\
1.420 \\
1.359 \\
\end{array}$ & 1.416 & 1.390 & 0.027 & 0.0162 \\
\hline & \multirow{3}{*}{ BTI2 } & \multirow{3}{*}{0.186} & Neutral & $\begin{array}{l}C^{16}-C^{17} \\
C^{13}-C^{15} \\
C^{11}-C^{12} \\
C^{10}-C^{39} \\
C^{34}-C^{38} \\
C^{32}-C^{33}\end{array}$ & $\begin{array}{l}1.432 \\
1.434 \\
1.426 \\
1.426 \\
1.434 \\
1.432\end{array}$ & $\begin{array}{l}\mathrm{C}^{17}=\mathrm{C}^{18} \\
\mathrm{C}^{15}=\mathrm{C}^{16} \\
\mathrm{C}^{12}=\mathrm{C}^{13} \\
\mathrm{C}^{10}=\mathrm{C}^{11} \\
\mathrm{C}^{38}=\mathrm{C}^{39} \\
\mathrm{C}^{33}=\mathrm{C}^{34} \\
\mathrm{C}^{31}=\mathrm{C}^{32}\end{array}$ & $\begin{array}{l}1.363 \\
1.389 \\
1.390 \\
1.381 \\
1.390 \\
1.389 \\
1.363 \\
\end{array}$ & 1.431 & 1.381 & 0.050 & -- \\
\hline & & & Cation & $\begin{array}{l}C^{16}-C^{17} \\
C^{13}-C^{15} \\
C^{11}-C^{12} \\
C^{10}-C^{39} \\
C^{34}-C^{38} \\
C^{32}-C^{33}\end{array}$ & $\begin{array}{l}1.414 \\
1.408 \\
1.397 \\
1.397 \\
1.408 \\
1.414\end{array}$ & $\begin{array}{l}\mathrm{C}^{17}=\mathrm{C}^{18} \\
\mathrm{C}^{15}=\mathrm{C}^{16} \\
\mathrm{C}^{12}=\mathrm{C}^{13} \\
\mathrm{C}^{10}=\mathrm{C}^{11} \\
\mathrm{C}^{38}=\mathrm{C}^{39} \\
\mathrm{C}^{33}=\mathrm{C}^{34} \\
\mathrm{C}^{31}=\mathrm{C}^{32}\end{array}$ & \begin{tabular}{l|}
1.378 \\
1.406 \\
1.413 \\
1.415 \\
1.413 \\
1.406 \\
1.378
\end{tabular} & 1.406 & 1.401 & 0.005 & 0.0110 \\
\hline & & & Anion & $\begin{array}{l}C^{16}-C^{17} \\
C^{13}-C^{15} \\
C^{11}-C^{12} \\
C^{10}-C^{39} \\
C^{34}-C^{38} \\
C^{32}-C^{33}\end{array}$ & $\begin{array}{l}1.436 \\
1.407 \\
1.411 \\
1.411 \\
1.407 \\
1.436\end{array}$ & $\begin{array}{l}\mathrm{C}^{17}=\mathrm{C}^{18} \\
\mathrm{C}^{15}=\mathrm{C}^{16} \\
\mathrm{C}^{12}=\mathrm{C}^{13} \\
\mathrm{C}^{10}=\mathrm{C}^{11} \\
\mathrm{C}^{38}=\mathrm{C}^{39} \\
\mathrm{C}^{33}=\mathrm{C}^{34} \\
\mathrm{C}^{31}=\mathrm{C}^{32}\end{array}$ & \begin{tabular}{l|l|}
1.363 & \\
1.411 \\
1.422 \\
1.401 \\
1.422 \\
1.411 \\
1.363
\end{tabular} & 1.414 & 1.396 & 0.018 & 0.0112 \\
\hline & \multirow{3}{*}{ ВTI3 } & \multirow{3}{*}{$\begin{array}{c}0.159 \\
3\end{array}$} & Neutral & $\begin{array}{l}C^{39}-C^{54} \\
C^{51}-C^{53} \\
C^{18}-C^{50} \\
C^{16}-C^{17} \\
C^{13}-C^{15} \\
C^{11}-C^{12} \\
C^{10}-C^{37} \\
C^{32}-C^{36} \\
C^{30}-C^{31}\end{array}$ & $\begin{array}{l}1.432 \\
1.432 \\
1.426 \\
1.424 \\
1.430 \\
1.425 \\
1.426 \\
1.432 \\
1.432\end{array}$ & $\begin{array}{l}\mathrm{C}^{39}=\mathrm{C}^{40} \\
\mathrm{C}^{53}=\mathrm{C}^{54} \\
\mathrm{C}^{50}=\mathrm{C}^{51} \\
\mathrm{C}^{17}=\mathrm{C}^{18} \\
\mathrm{C}^{15}=\mathrm{C}^{16} \\
\mathrm{C}^{12}=\mathrm{C}^{13} \\
\mathrm{C}^{10}=\mathrm{C}^{11} \\
\mathrm{C}^{36}=\mathrm{C}^{37} \\
\mathrm{C}^{31}=\mathrm{C}^{32} \\
\mathrm{C}^{29}=\mathrm{C}^{30}\end{array}$ & $\begin{array}{l}1.365 \\
1.392 \\
1.393 \\
1.384 \\
1.396 \\
1.397 \\
1.384 \\
1.393 \\
1.392 \\
1.365 \\
\end{array}$ & 1.429 & 1.386 & 0.043 & -- \\
\hline & & & Cation & $\begin{array}{l}C^{39}-C^{54} \\
C^{51}-C^{53} \\
C^{18}-C^{50} \\
C^{16}-C^{17} \\
C^{13}-C^{15} \\
C^{11}-C^{12} \\
C^{10}-C^{37} \\
C^{32}-C^{36} \\
C^{30}-C^{31}\end{array}$ & $\begin{array}{l}1.421 \\
1.418 \\
1.408 \\
1.399 \\
1.398 \\
1.400 \\
1.408 \\
1.418 \\
1.421\end{array}$ & $\begin{array}{l}\mathrm{C}^{39}=\mathrm{C}^{40} \\
\mathrm{C}^{53}=\mathrm{C}^{54} \\
\mathrm{C}^{50}=\mathrm{C}^{51} \\
\mathrm{C}^{17}=\mathrm{C}^{18} \\
\mathrm{C}^{15}=\mathrm{C}^{16} \\
\mathrm{C}^{12}=\mathrm{C}^{13} \\
\mathrm{C}^{10}=\mathrm{C}^{11} \\
\mathrm{C}^{36}=\mathrm{C}^{37} \\
\mathrm{C}^{31}=\mathrm{C}^{32} \\
\mathrm{C}^{29}=\mathrm{C}^{30}\end{array}$ & $\begin{array}{l}1.373 \\
1.401 \\
1.405 \\
1.409 \\
1.419 \\
1.420 \\
1.409 \\
1.405 \\
1.401 \\
1.373 \\
\end{array}$ & 1.410 & 1.401 & 0.009 & 0.0084 \\
\hline & & & Anion & $\begin{array}{l}C^{39}-C^{54} \\
C^{51}-C^{53} \\
C^{18}-C^{50} \\
C^{16}-C^{17} \\
C^{13}-C^{15} \\
C^{11}-C^{12} \\
C^{10}-C^{37} \\
C^{32}-C^{36} \\
C^{30}-C^{31}\end{array}$ & $\begin{array}{l}1.435 \\
1.416 \\
1.414 \\
1.412 \\
1.392 \\
1.413 \\
1.414 \\
1.416 \\
1.435\end{array}$ & $\begin{array}{l}\mathrm{C}^{39}=\mathrm{C}^{40} \\
\mathrm{C}^{53}=\mathrm{C}^{54} \\
\mathrm{C}^{50}=\mathrm{C}^{51} \\
\mathrm{C}^{17}=\mathrm{C}^{18} \\
\mathrm{C}^{15}=\mathrm{C}^{16} \\
\mathrm{C}^{12}=\mathrm{C}^{13} \\
\mathrm{C}^{10}=\mathrm{C}^{11} \\
\mathrm{C}^{36}=\mathrm{C}^{37} \\
\mathrm{C}^{31}=\mathrm{C}^{32} \\
\mathrm{C}^{29}=\mathrm{C}^{30}\end{array}$ & $\begin{array}{l}1.362 \\
1.400 \\
1.409 \\
1.395 \\
1.424 \\
1.426 \\
1.394 \\
1.408 \\
1.400 \\
1.362 \\
\end{array}$ & 1.416 & 1.398 & 0.018 & 0.0083 \\
\hline & BTI4 & $\begin{array}{c}0.142 \\
9\end{array}$ & Neutral & $\begin{array}{l}C^{56}-C^{71} \\
C^{68}-C^{70} \\
C^{37}-C^{67} \\
C^{35}-C^{36} \\
C^{32}-C^{34} \\
C^{30}-C^{31} \\
C^{29}-C^{54} \\
C^{51}-C^{53} \\
C^{49}-C^{50} \\
\end{array}$ & $\begin{array}{l}1.432 \\
1.431 \\
1.426 \\
1.424 \\
1.429 \\
1.425 \\
1.425 \\
1.429 \\
1.424 \\
\end{array}$ & $\begin{array}{l}\mathrm{C}^{56}=\mathrm{C}^{57} \\
\mathrm{C}^{70}=\mathrm{C}^{71} \\
\mathrm{C}^{67}=\mathrm{C}^{68} \\
\mathrm{C}^{36}=\mathrm{C}^{37} \\
\mathrm{C}^{34}=\mathrm{C}^{35} \\
\mathrm{C}^{31}=\mathrm{C}^{32} \\
\mathrm{C}^{29}=\mathrm{C}^{30} \\
\mathrm{C}^{50}=\mathrm{C}^{51} \\
\mathrm{C}^{53}=\mathrm{C}^{54}\end{array}$ & $\begin{array}{l}1.366 \\
1.394 \\
1.395 \\
1.385 \\
1.398 \\
1.399 \\
1.386 \\
1.398 \\
1.399 \\
\end{array}$ & 1.428 & 1.389 & 0.039 & -- \\
\hline
\end{tabular}




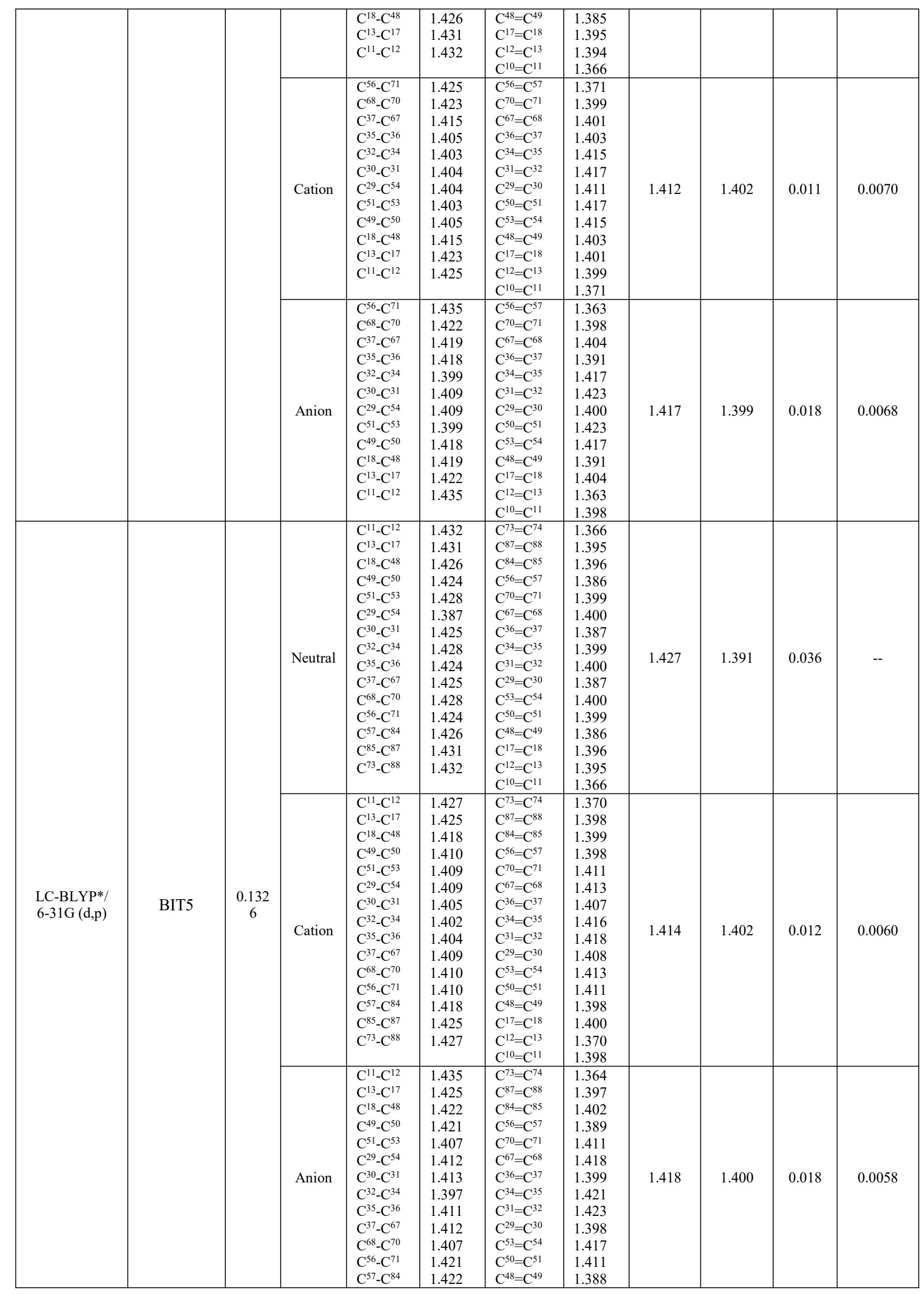




\begin{tabular}{|c|c|c|c|c|c|c|c|c|c|c|c|}
\hline & & & & $\begin{array}{l}C^{85}-C^{87} \\
C^{73}-C^{88}\end{array}$ & $\begin{array}{l}1.425 \\
1.435\end{array}$ & $\begin{array}{l}\mathrm{C}^{17}=\mathrm{C}^{18} \\
\mathrm{C}^{12}=\mathrm{C}^{13} \\
\mathrm{C}^{10}=\mathrm{C}^{11}\end{array}$ & $\begin{array}{l}1.402 \\
1.397 \\
1.364 \\
\end{array}$ & & & & \\
\hline \multirow{4}{*}{$\begin{array}{l}\text { LC-BLYP*/ } \\
\text { 6-31G (d,p) }\end{array}$} & \multirow{3}{*}{ BIT6 } & \multirow{3}{*}{$\begin{array}{c}0.125 \\
0\end{array}$} & Neutral & 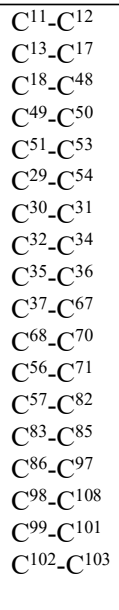 & $\begin{array}{l}1.433 \\
1.430 \\
1.426 \\
1.424 \\
1.427 \\
1.425 \\
1.424 \\
1.427 \\
1.425 \\
1.424 \\
1.427 \\
1.425 \\
1.425 \\
1.427 \\
1.424 \\
1.426 \\
1.430 \\
1.433\end{array}$ & $\begin{array}{l}\mathrm{C}^{10}=\mathrm{C}^{11} \\
\mathrm{C}^{12}=\mathrm{C}^{13} \\
\mathrm{C}^{17}=\mathrm{C}^{18} \\
\mathrm{C}^{48}=\mathrm{C}^{49} \\
\mathrm{C}^{50}=\mathrm{C}^{51} \\
\mathrm{C}^{53}=\mathrm{C}^{54} \\
\mathrm{C}^{29}=\mathrm{C}^{30} \\
\mathrm{C}^{31}=\mathrm{C}^{32} \\
\mathrm{C}^{34}=\mathrm{C}^{35} \\
\mathrm{C}^{36}=\mathrm{C}^{37} \\
\mathrm{C}^{67}=\mathrm{C}^{68} \\
\mathrm{C}^{70}=\mathrm{C}^{71} \\
\mathrm{C}^{56}=\mathrm{C}^{57} \\
\mathrm{C}^{82}=\mathrm{C}^{83} \\
\mathrm{C}^{85}=\mathrm{C}^{86} \\
\mathrm{C}^{97}=\mathrm{C}^{100} \\
\mathrm{C}^{98}=\mathrm{C}^{99} \\
\mathrm{C}^{101}=\mathrm{C}^{102} \\
\mathrm{C}^{103}=\mathrm{C}^{104}\end{array}$ & $\begin{array}{l}1.367 \\
1.395 \\
1.397 \\
1.387 \\
1.400 \\
1.401 \\
1.387 \\
1.400 \\
1.401 \\
1.387 \\
1.400 \\
1.401 \\
1.387 \\
1.401 \\
1.400 \\
1.387 \\
1.397 \\
1.395 \\
1.367\end{array}$ & 1.427 & 1.392 & 0.034 & -- \\
\hline & & & Cation & 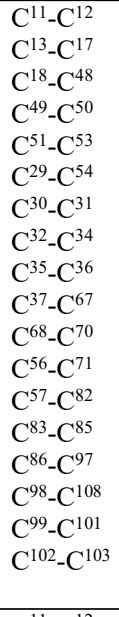 & $\begin{array}{l}1.429 \\
1.427 \\
1.421 \\
1.414 \\
1.414 \\
1.413 \\
1.407 \\
1.405 \\
1.407 \\
1.406 \\
1.405 \\
1.408 \\
1.413 \\
1.414 \\
1.414 \\
1.421 \\
1.427 \\
1.429\end{array}$ & $\begin{array}{l}\mathrm{C}^{10}=\mathrm{C}^{11} \\
\mathrm{C}^{12}=\mathrm{C}^{13} \\
\mathrm{C}^{17}=\mathrm{C}^{18} \\
\mathrm{C}^{48}=\mathrm{C}^{49} \\
\mathrm{C}^{50}=\mathrm{C}^{51} \\
\mathrm{C}^{53}=\mathrm{C}^{54} \\
\mathrm{C}^{29}=\mathrm{C}^{30} \\
\mathrm{C}^{31}=\mathrm{C}^{32} \\
\mathrm{C}^{33}=\mathrm{C}^{35} \\
\mathrm{C}^{36}=\mathrm{C}^{37} \\
\mathrm{C}^{67}=\mathrm{C}^{68} \\
\mathrm{C}^{70}=\mathrm{C}^{71} \\
\mathrm{C}^{56}=\mathrm{C}^{57} \\
\mathrm{C}^{82}=\mathrm{C}^{83} \\
\mathrm{C}^{85}=\mathrm{C}^{86} \\
\mathrm{C}^{97}=\mathrm{C}^{100} \\
\mathrm{C}^{98}=\mathrm{C}^{99} \\
\mathrm{C}^{101}=\mathrm{C}^{102} \\
\mathrm{C}^{103}=\mathrm{C}^{104}\end{array}$ & $\begin{array}{l}1.370 \\
1.398 \\
1.399 \\
1.395 \\
1.408 \\
1.410 \\
1.404 \\
1.414 \\
1.416 \\
1.408 \\
1.415 \\
1.416 \\
1.404 \\
1.410 \\
1.408 \\
1.395 \\
1.399 \\
1.398 \\
1.370\end{array}$ & 1.415 & 1.402 & 0.013 & 0.0053 \\
\hline & & & Anion & 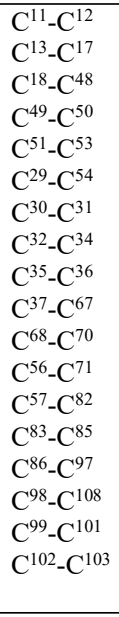 & $\begin{array}{l}1.435 \\
1.426 \\
1.423 \\
1.423 \\
1.413 \\
1.415 \\
1.415 \\
1.401 \\
1.411 \\
1.410 \\
1.401 \\
1.417 \\
1.415 \\
1.414 \\
1.423 \\
1.423 \\
1.427 \\
1.434\end{array}$ & $\begin{array}{l}\mathrm{C}^{10}=\mathrm{C}^{11} \\
\mathrm{C}^{12}=\mathrm{C}^{13} \\
\mathrm{C}^{17}=\mathrm{C}^{18} \\
\mathrm{C}^{48}=\mathrm{C}^{49} \\
\mathrm{C}^{50}=\mathrm{C}^{51} \\
\mathrm{C}^{53}=\mathrm{C}^{54} \\
\mathrm{C}^{29}=\mathrm{C}^{30} \\
\mathrm{C}^{31}=\mathrm{C}^{32} \\
\mathrm{C}^{34}=\mathrm{C}^{35} \\
\mathrm{C}^{36}=\mathrm{C}^{37} \\
\mathrm{C}^{67}=\mathrm{C}^{68} \\
\mathrm{C}^{70}=\mathrm{C}^{71} \\
\mathrm{C}^{56}=\mathrm{C}^{57} \\
\mathrm{C}^{82}=\mathrm{C}^{83} \\
\mathrm{C}^{85}=\mathrm{C}^{86} \\
\mathrm{C}^{97}=\mathrm{C}^{100} \\
\mathrm{C}^{98}=\mathrm{C}^{99} \\
\mathrm{C}^{101}=\mathrm{C}^{102} \\
\mathrm{C}^{103}=\mathrm{C}^{104}\end{array}$ & $\begin{array}{l}1.365 \\
1.397 \\
1.401 \\
1.388 \\
1.408 \\
1.413 \\
1.396 \\
1.418 \\
1.421 \\
1.400 \\
1.419 \\
1.419 \\
1.395 \\
1.413 \\
1.407 \\
1.388 \\
1.401 \\
1.397 \\
1.365 \\
\end{array}$ & 1.418 & 1.400 & 0.018 & 0.0051 \\
\hline & BIT7 & $\begin{array}{c}0.119 \\
8\end{array}$ & Neutral & $\begin{array}{l}\mathrm{C}^{11}-\mathrm{C}^{12} \\
\mathrm{C}^{13}-\mathrm{C}^{17} \\
\mathrm{C}^{18}-\mathrm{C}^{84} \\
\mathrm{C}^{49}-\mathrm{C}^{50} \\
\mathrm{C}^{515}-\mathrm{C}^{53} \\
\mathrm{C}^{29}-\mathrm{C}^{54} \\
\mathrm{C}^{30}-\mathrm{C}^{31} \\
\mathrm{C}^{32}-\mathrm{C}^{34} \\
\mathrm{C}^{35}-\mathrm{C}^{36} \\
\mathrm{C}^{37} \mathrm{C}^{67} \\
\mathrm{C}^{68}-\mathrm{C}^{70} \\
\mathrm{C}^{56}-\mathrm{C}^{71} \\
\mathrm{C}^{57}-\mathrm{C}^{82} \\
\mathrm{C}^{83}-\mathrm{C}^{85} \\
\mathrm{C}^{86}-\mathrm{C}^{97}\end{array}$ & $\begin{array}{l}1.433 \\
1.430 \\
1.426 \\
1.424 \\
1.427 \\
1.425 \\
1.424 \\
1.427 \\
1.425 \\
1.425 \\
1.427 \\
1.424 \\
1.424 \\
1.427 \\
1.425\end{array}$ & $\begin{array}{l}\mathrm{C}^{10}=\mathrm{C}^{11} \\
\mathrm{C}^{12}=\mathrm{C}^{13} \\
\mathrm{C}^{17}=\mathrm{C}^{18} \\
\mathrm{C}^{48}=\mathrm{C}^{49} \\
\mathrm{C}^{50}=\mathrm{C}^{51} \\
\mathrm{C}^{53}=\mathrm{C}^{54} \\
\mathrm{C}^{29}=\mathrm{C}^{30} \\
\mathrm{C}^{31}=\mathrm{C}^{32} \\
\mathrm{C}^{34}=\mathrm{C}^{35} \\
\mathrm{C}^{36}=\mathrm{C}^{37} \\
\mathrm{C}^{67}=\mathrm{C}^{68} \\
\mathrm{C}^{70}=\mathrm{C}^{71} \\
\mathrm{C}^{56}=\mathrm{C}^{57} \\
\mathrm{C}^{82}=\mathrm{C}^{83} \\
\mathrm{C}^{85}=\mathrm{C}^{86}\end{array}$ & $\begin{array}{l}1.367 \\
1.396 \\
1.398 \\
1.387 \\
1.401 \\
1.402 \\
1.388 \\
1.400 \\
1.402 \\
1.388 \\
1.402 \\
1.400 \\
1.388 \\
1.400 \\
1.402\end{array}$ & 1.426 & 1394 & 0.033 & -- \\
\hline
\end{tabular}




\begin{tabular}{|c|c|c|c|c|c|c|c|c|c|c|c|}
\hline & & & & $\begin{array}{l}\mathrm{C}^{98}-\mathrm{C}^{106} \\
\mathrm{C}^{99}-\mathrm{C}^{101} \\
\mathrm{C}^{102}-\mathrm{C}^{103} \\
\mathrm{C}^{104}-\mathrm{C}^{124} \\
\mathrm{C}^{119} \mathrm{C}^{123} \\
\mathrm{C}^{117} \mathrm{C}^{118} \mathrm{C}^{118}\end{array}$ & $\begin{array}{l}1.425 \\
1.427 \\
1.424 \\
1.426 \\
1.430 \\
1.433\end{array}$ & $\begin{array}{l}C^{97}=C^{106} \\
C^{98}=C^{99} \\
C^{101}=C^{102} \\
C^{103}=C^{104} \\
C^{123}-C^{124} \\
C^{118}-C^{119} \\
C^{116}-C^{117}\end{array}$ & $\begin{array}{l}1.388 \\
1.402 \\
1.401 \\
1.387 \\
1.398 \\
1.396 \\
1.367\end{array}$ & & & & \\
\hline \multirow{3}{*}{$\begin{array}{l}\text { LC-BLYP*/ } \\
\text { 6-31G (d,p) }\end{array}$} & & & Cation & 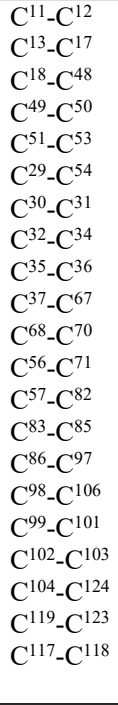 & $\begin{array}{l}1.430 \\
1.428 \\
1.422 \\
1.416 \\
1.418 \\
1.417 \\
1.410 \\
1.409 \\
1.410 \\
1.407 \\
1.405 \\
1.406 \\
1.409 \\
1.409 \\
1.411 \\
1.417 \\
1.418 \\
1.416 \\
1.422 \\
1.428 \\
1.430\end{array}$ & $\begin{array}{l}C^{10}=C^{11} \\
C^{11}=C^{13} \\
C^{17}=C^{18} \\
C^{48}=C^{49} \\
C^{50}=C^{51} \\
C^{53}=C^{54} \\
C^{29}=C^{30} \\
C^{31}=C^{32} \\
C^{34}=C^{35} \\
C^{36}=C^{37} \\
C^{67}=C^{68} \\
C^{77}=C^{71} \\
C^{56}=C^{57} \\
C^{82}=C^{83} \\
C^{85}=C^{86} \\
C^{97}=C^{106} \\
C^{98}=C^{99} \\
C^{101}=C^{102} \\
C^{103}=C^{104} \\
C^{123}-C^{124} \\
C^{118}-C^{119} \\
C^{116}-C^{117}\end{array}$ & $\begin{array}{l}1.369 \\
1.398 \\
1.399 \\
1.393 \\
1.407 \\
1.407 \\
1.400 \\
1.412 \\
1.413 \\
1.406 \\
1.417 \\
1.415 \\
1.406 \\
1.412 \\
1.413 \\
1.400 \\
1.407 \\
1.407 \\
1.393 \\
1.399 \\
1.398 \\
1.369 \\
\end{array}$ & 1.416 & 1.402 & 0.014 & 0.0047 \\
\hline & & & Anion & 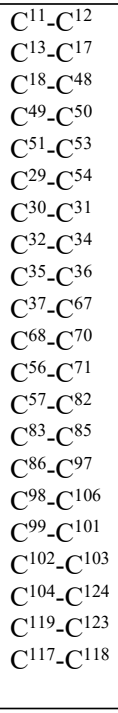 & $\begin{array}{l}1.434 \\
1.427 \\
1.424 \\
1.424 \\
1.417 \\
1.418 \\
1.419 \\
1.406 \\
1.413 \\
1.413 \\
1.400 \\
1.412 \\
1.411 \\
1.406 \\
1.420 \\
1.418 \\
1.417 \\
1.424 \\
1.424 \\
1.427 \\
1.434\end{array}$ & $\begin{array}{l}\mathrm{C}^{10}=\mathrm{C}^{11} \\
\mathrm{C}^{12}=\mathrm{C}^{13} \\
\mathrm{C}^{17}=\mathrm{C}^{11} \\
\mathrm{C}^{48}=\mathrm{C}^{49} \\
\mathrm{C}^{50}=\mathrm{C}^{51} \\
\mathrm{C}^{53}=\mathrm{C}^{54} \\
\mathrm{C}^{29}=\mathrm{C}^{30} \\
\mathrm{C}^{31}=\mathrm{C}^{32} \\
\mathrm{C}^{34}=\mathrm{C}^{35} \\
\mathrm{C}^{36}=\mathrm{C}^{37} \\
\mathrm{C}^{67}=\mathrm{C}^{68} \\
\mathrm{C}^{70}=\mathrm{C}^{71} \\
\mathrm{C}^{56}=\mathrm{C}^{57} \\
\mathrm{C}^{82}=\mathrm{C}^{83} \\
\mathrm{C}^{85}=\mathrm{C}^{86} \\
\mathrm{C}^{97}=\mathrm{C}^{106} \\
\mathrm{C}^{98}=\mathrm{C}^{99} \\
\mathrm{C}^{101}=\mathrm{C}^{102} \\
\mathrm{C}^{103}=\mathrm{C}^{104} \\
\mathrm{C}^{123}-\mathrm{C}^{124} \\
\mathrm{C}^{118}-\mathrm{C}^{119} \\
\mathrm{C}^{116}-\mathrm{C}^{117}\end{array}$ & $\begin{array}{l}1.366 \\
1.397 \\
1.400 \\
1.387 \\
1.405 \\
1.410 \\
1.393 \\
1.413 \\
1.418 \\
1.399 \\
1.421 \\
1.420 \\
1.399 \\
1.417 \\
1.415 \\
1.393 \\
1.410 \\
1.405 \\
1.387 \\
1.400 \\
1.397 \\
1.366 \\
\end{array}$ & 1.419 & 1.401 & 0.018 & 0.0045 \\
\hline & BIT8 & $\begin{array}{c}0.115 \\
7\end{array}$ & Neutral & 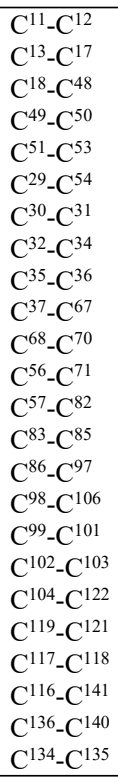 & $\begin{array}{l}1.433 \\
1.429 \\
1.426 \\
1.424 \\
1.426 \\
1.425 \\
1.424 \\
1.426 \\
1.425 \\
1.425 \\
1.426 \\
1.424 \\
1.424 \\
1.426 \\
1.425 \\
1.424 \\
1.426 \\
1.425 \\
1.425 \\
1.426 \\
1.424 \\
1.426 \\
1.429 \\
1.433 \\
\end{array}$ & $\begin{array}{l}\mathrm{C}^{10}=\mathrm{C}^{11} \\
\mathrm{C}^{12}=\mathrm{C}^{13} \\
\mathrm{C}^{17}=\mathrm{C}^{18} \\
\mathrm{C}^{48}=\mathrm{C}^{49} \\
\mathrm{C}^{50}=\mathrm{C}^{51} \\
\mathrm{C}^{53}=\mathrm{C}^{54} \\
\mathrm{C}^{29}=\mathrm{C}^{30} \\
\mathrm{C}^{31}=\mathrm{C}^{32} \\
\mathrm{C}^{33}=\mathrm{C}^{33} \\
\mathrm{C}^{36}=\mathrm{C}^{37} \\
\mathrm{C}^{67}=\mathrm{C}^{68} \\
\mathrm{C}^{70}=\mathrm{C}^{71} \\
\mathrm{C}^{56}=\mathrm{C}^{57} \\
\mathrm{C}^{88}=\mathrm{C}^{83} \\
\mathrm{C}^{85}=\mathrm{C}^{86} \\
\mathrm{C}^{97}=\mathrm{C}^{106} \\
\mathrm{C}^{98}=\mathrm{C}^{99} \\
\mathrm{C}^{101}=\mathrm{C}^{102} \\
\mathrm{C}^{103}=\mathrm{C}^{104} \\
\mathrm{C}^{1118} 8 \mathrm{C}^{119} \\
\mathrm{C}^{121}-\mathrm{C}^{122} \\
\mathrm{C}^{116}-\mathrm{C}^{117} \\
\mathrm{C}^{140}-\mathrm{C}^{141} \\
\mathrm{C}^{133}-\mathrm{C}^{134}\end{array}$ & $\begin{array}{l}1.367 \\
1.396 \\
1.398 \\
1.388 \\
1.401 \\
1.402 \\
1.388 \\
1.401 \\
1.402 \\
1.388 \\
1.402 \\
1.401 \\
1.388 \\
1.401 \\
1.402 \\
1.388 \\
1.401 \\
1.402 \\
1.388 \\
1.402 \\
1.401 \\
1.388 \\
1.398 \\
1.396 \\
\end{array}$ & 1.426 & 1.394 & 0.032 & -- \\
\hline
\end{tabular}




\begin{tabular}{|c|c|c|c|c|c|c|c|c|c|c|}
\hline & & & & & $\mathrm{C}^{135}-\mathrm{C}^{136}$ & 1.367 & & & & \\
\hline \multirow{47}{*}{$\begin{array}{l}\text { LC-BLYP*/ } \\
6-31 G(d, p)\end{array}$} & & \multirow{24}{*}{ Cation } & $\mathrm{C}^{11}-\mathrm{C}^{12}$ & 1.430 & $\mathrm{C}^{10}=\mathrm{C}^{11}$ & 1.369 & \multirow{24}{*}{1.417} & \multirow{24}{*}{1.402} & \multirow{24}{*}{0.015} & \multirow{24}{*}{0.0043} \\
\hline & & & $\mathrm{C}^{13}-\mathrm{C}^{17}$ & 1.428 & $\mathrm{C}^{12}=\mathrm{C}^{13}$ & 1.398 & & & & \\
\hline & & & $\mathrm{C}^{18}-\mathrm{C}^{48}$ & 1.423 & $\mathrm{C}^{17}=\mathrm{C}^{18}$ & 1.399 & & & & \\
\hline & & & $C^{49}-C^{50}$ & 1.418 & $C^{48}=C^{49}$ & 1.392 & & & & \\
\hline & & & $C^{51}-C^{53}$ & 1.420 & $C^{50}=C^{51}$ & 1.405 & & & & \\
\hline & & & $\mathrm{C}^{29}-\mathrm{C}^{54}$ & 1.419 & $\mathrm{C}^{53}=\mathrm{C}^{54}$ & 1.406 & & & & \\
\hline & & & $\begin{array}{l}C^{30}-C^{31} \\
C^{32}-C^{34}\end{array}$ & $\begin{array}{l}1.413 \\
1.413\end{array}$ & $\begin{array}{l}\mathrm{C}^{29}=\mathrm{C}^{30} \\
\mathrm{C}^{31}=\mathrm{C}^{32}\end{array}$ & $\begin{array}{l}1.397 \\
1.410\end{array}$ & & & & \\
\hline & & & $C^{35}-C^{36}$ & 1.413 & $\mathrm{C}^{34}=\mathrm{C}^{35}$ & 1.411 & & & & \\
\hline & & & $C^{37}-C^{67}$ & 1.409 & $\mathrm{C}^{36}=\mathrm{C}^{37}$ & 1.403 & & & & \\
\hline & & & $C^{68}-C^{70}$ & 1.407 & $C^{67}=C^{68}$ & 1.415 & & & & \\
\hline & & & $C^{56}-C^{71}$ & 1.408 & $\mathrm{C}^{70}=\mathrm{C}^{71}$ & 1.414 & & & & \\
\hline & & & $C^{57}-C^{82}$ & 1.408 & $C^{56}=C^{57}$ & 1.406 & & & & \\
\hline & & & $C^{83}-C^{85}$ & 1.407 & $C^{82}=C^{83}$ & 1.414 & & & & \\
\hline & & & $\mathrm{C}^{86}-\mathrm{C}^{97}$ & 1.409 & $C^{85}=C^{86}$ & 1.415 & & & & \\
\hline & & & $\mathrm{C}^{98}-\mathrm{C}^{106}$ & 1.412 & $\mathrm{C}^{97}=\mathrm{C}^{106}$ & 1.403 & & & & \\
\hline & & & $\mathrm{C}^{99}-\mathrm{C}^{101}$ & 1.413 & $\mathrm{C}^{98}=\mathrm{C}^{99}$ & 1.409 & & & & \\
\hline & & & $\mathrm{C}^{102}-\mathrm{C}^{103}$ & 1.414 & $\mathrm{C}^{101}=\mathrm{C}^{102}$ & 1.411 & & & & \\
\hline & & & $C^{104}-C^{122}$ & 1.419 & $C^{103}=C^{104}$ & 1.398 & & & & \\
\hline & & & $C^{119}-C^{121}$ & 1.420 & $C^{118}-C^{119}$ & 1.406 & & & & \\
\hline & & & $C^{117}-C^{118}$ & 1.418 & $C^{121}-C^{122}$ & 1.418 & & & & \\
\hline & & & $C^{116}-C^{141}$ & 1.423 & $C^{116}-C^{117}$ & 1.392 & & & & \\
\hline & & & $C^{136}-C^{140}$ & 1.428 & $C^{140}-C^{141}$ & 1.399 & & & & \\
\hline & & & $\mathrm{C}^{134}-\mathrm{C}^{135}$ & 1.430 & $\mathrm{C}^{133}-\mathrm{C}^{134}$ & 1.398 & & & & \\
\hline & & & & & $C^{135}-C^{136}$ & 1.369 & & & & \\
\hline & & & $\mathrm{C}^{11}-\mathrm{C}^{12}$ & 1.434 & $\mathrm{C}^{10}=\mathrm{C}^{11}$ & 1.366 & \multirow{23}{*}{1.419} & \multirow{23}{*}{1.401} & & \\
\hline & & & $\mathrm{C}^{13}-\mathrm{C}^{17}$ & 1.428 & $\mathrm{C}^{12}=\mathrm{C}^{13}$ & 1.397 & & & & \\
\hline & & & $\begin{array}{l}\mathrm{C}^{10}-\mathrm{C}^{40} \\
C^{49} C^{50}\end{array}$ & 1.425 & $\begin{array}{l}\mathrm{C}^{11}=\mathrm{C}^{10} \\
C^{48}=C^{49}\end{array}$ & 1.400 & & & & \\
\hline & & & $C^{51}-C^{53}$ & 1.420 & $\mathrm{C}^{50}=\mathrm{C}^{51}$ & 1.404 & & & & \\
\hline & & & $C^{29}-C^{54}$ & 1.420 & $\mathrm{C}^{53}=\mathrm{C}^{54}$ & 1.408 & & & & \\
\hline & & & $\mathrm{C}^{30}-\mathrm{C}^{31}$ & 1.421 & $\mathrm{C}^{29}=\mathrm{C}^{30}$ & 1.391 & & & & \\
\hline & & & $\mathrm{C}^{32}-\mathrm{C}^{34}$ & 1.411 & $\mathrm{C}^{31}=\mathrm{C}^{32}$ & 1.410 & & & & \\
\hline & & & $C^{35}-C^{36}$ & 1.415 & $\mathrm{C}^{34}=\mathrm{C}^{35}$ & 1.414 & & & & \\
\hline & & & $C^{37}-C^{67}$ & 1.416 & $\mathrm{C}^{36}=\mathrm{C}^{37}$ & 1.396 & & & & \\
\hline & & & $\mathrm{C}^{68}-\mathrm{C}^{70}$ & 1.402 & $C^{67}=C^{68}$ & 1.419 & & & & \\
\hline & & & $C^{56}-C^{71}$ & 1.411 & $\mathrm{C}^{70}=\mathrm{C}^{71}$ & 1.419 & & & & \\
\hline & & Anion & $C^{57}-C^{82}$ & 1.411 & $C^{56}=C^{57}$ & 1.400 & & & 0.018 & 0.0041 \\
\hline & & & $C^{83}-C^{85}$ & 1.402 & $C^{82}=C^{83}$ & 1.419 & & & & \\
\hline & & & $C^{86}-C^{97}$ & 1.416 & $\mathrm{C}^{85}=\mathrm{C}^{86}$ & 1.419 & & & & \\
\hline & & & $\mathrm{C}^{98}-\mathrm{C}^{106}$ & 1.414 & $\mathrm{C}^{97}=\mathrm{C}^{106}$ & 1.397 & & & & \\
\hline & & & $C^{99}-C^{101}$ & 1.411 & $\mathrm{C}^{98}=\mathrm{C}^{99}$ & 1.413 & & & & \\
\hline & & & $\mathrm{C}^{102}-\mathrm{C}^{103}$ & 1.422 & $\mathrm{C}^{101}=\mathrm{C}^{102}$ & 1.411 & & & & \\
\hline & & & $C^{104}-C^{122}$ & 1.420 & $C^{103}=C^{104}$ & 1.391 & & & & \\
\hline & & & $C^{119}-C^{121}$ & 1.420 & $C^{118}-C^{119}$ & 1.408 & & & & \\
\hline & & & $C^{117}-C^{118}$ & 1.424 & $C^{121}-C^{122}$ & 1.404 & & & & \\
\hline & & & $C^{116}-C^{141}$ & 1.425 & $C^{116}-C^{117}$ & 1.387 & & & & \\
\hline & & & $C^{136}-C^{140}$ & 1.428 & $C^{140}-C^{141}$ & 1.400 & & & & \\
\hline & & & $C^{134}-C^{135}$ & 1.434 & $\begin{array}{l}C^{133}-C^{134} \\
C^{135} C^{136}\end{array}$ & 1.397 & & & & \\
\hline & & & $\mathrm{C}^{11}-\mathrm{C}^{12}$ & 1.434 & $\mathrm{C}^{10}=\mathrm{C}^{11}$ & 1.360 & & & & \\
\hline & & Neutral & $\mathrm{C}^{13}-\mathrm{C}^{17}$ & 1.434 & $\mathrm{C}^{12}=\mathrm{C}^{13}$ & 1.388 & 1434 & 1374 & 0060 & $\ldots$ \\
\hline & & Neutral & $\mathrm{C}^{18}-\mathrm{C}^{19}$ & 1.434 & $\begin{array}{l}\mathrm{C}^{17}=\mathrm{C}^{18} \\
C^{19}=C^{20}\end{array}$ & 1.388 & 1.434 & $1.3 / 4$ & 0.060 & -- \\
\hline & & & $\mathrm{C}^{11} \mathrm{C}^{12}$ & 1.402 & $\mathrm{C}^{10}=\mathrm{C}^{11}$ & 1.389 & & & & \\
\hline & & & $\mathrm{C}^{13}-\mathrm{C}^{17}$ & 1.397 & $\mathrm{C}^{12}=\mathrm{C}^{13}$ & 1.420 & & & & \\
\hline & BTI & Cation & $\mathrm{C}^{18}-\mathrm{C}^{19}$ & 1.402 & $\mathrm{C}^{17}=\mathrm{C}^{18}$ & 1.420 & 1.400 & 1.404 & -0.004 & 0.0158 \\
\hline & & & & & $\mathrm{C}^{19}=\mathrm{C}^{20}$ & 1.389 & & & & \\
\hline & & & $\mathrm{C}^{11}-\mathrm{C}^{12}$ & 1.432 & $\mathrm{C}^{10}=\mathrm{C}^{11}$ & 1.359 & & & & \\
\hline & & Anion & $\mathrm{C}^{13}-\mathrm{C}^{17}$ & 1.390 & $\mathrm{C}^{12}=\mathrm{C}^{13}$ & 1.426 & 1418 & 1392 & 0025 & 00161 \\
\hline & & Anion & $\mathrm{C}^{18}-\mathrm{C}^{19}$ & 1.432 & $\mathrm{C}^{17}=\mathrm{C}^{18}$ & 1.426 & 1.418 & 1.392 & 0.025 & \\
\hline B3LYP/6-31G & & & & & $\mathrm{C}^{19}=\mathrm{C}^{20}$ & 1.359 & & & & \\
\hline$(\mathrm{d}, \mathrm{p})$ & & & $\mathrm{C}^{16}-\mathrm{C}^{17}$ & 1.433 & $\mathrm{C}^{17}=\mathrm{C}^{18}$ & 1.360 & & & & \\
\hline & & & $\mathrm{C}^{13}-\mathrm{C}^{15}$ & 1.430 & $\mathrm{C}^{15}=\mathrm{C}^{16}$ & 1.391 & & & & \\
\hline & & & $\mathrm{C}^{11}-\mathrm{C}^{12}$ & 1.426 & $\mathrm{C}^{12}=\mathrm{C}^{13}$ & 1.393 & & & & \\
\hline & & Neutral & $\mathrm{C}^{10}-\mathrm{C}^{39}$ & 1.426 & $\mathrm{C}^{10}=\mathrm{C}^{11}$ & 1.383 & 1.430 & 1.382 & 0.048 & -- \\
\hline & & & $C^{34}-C^{38}$ & 1.430 & $\mathrm{C}^{38}=\mathrm{C}^{39}$ & 1.393 & & & & \\
\hline & & & $\mathrm{C}^{32}-\mathrm{C}^{33}$ & 1.433 & $C^{33}=C^{34}$ & 1.391 & & & & \\
\hline & В112 & & & & $\mathrm{C}^{31}=\mathrm{C}^{32}$ & 1.360 & & & & \\
\hline & & & $\mathrm{C}^{16}-\mathrm{C}^{17}$ & 1.414 & $\mathrm{C}^{17}=\mathrm{C}^{18}$ & 1.376 & & & & \\
\hline & & & $\mathrm{C}^{13}-\mathrm{C}^{15}$ & 1.407 & $\mathrm{C}^{15}=\mathrm{C}^{16}$ & 1.407 & & & & \\
\hline & & Cation & $\mathrm{C}^{11}-\mathrm{C}^{12}$ & 1.400 & $\mathrm{C}^{12}=\mathrm{C}^{13}$ & 1.413 & 1.407 & 1.401 & 0.007 & 0.0105 \\
\hline & & & $\mathrm{C}^{10}-\mathrm{C}^{39}$ & 1.400 & $\mathrm{C}^{10}=\mathrm{C}^{11}$ & 1.413 & & & & \\
\hline & & & $C^{34}-C^{38}$ & 1.407 & $\mathrm{C}^{38}=\mathrm{C}^{39}$ & 1.413 & & & & \\
\hline
\end{tabular}




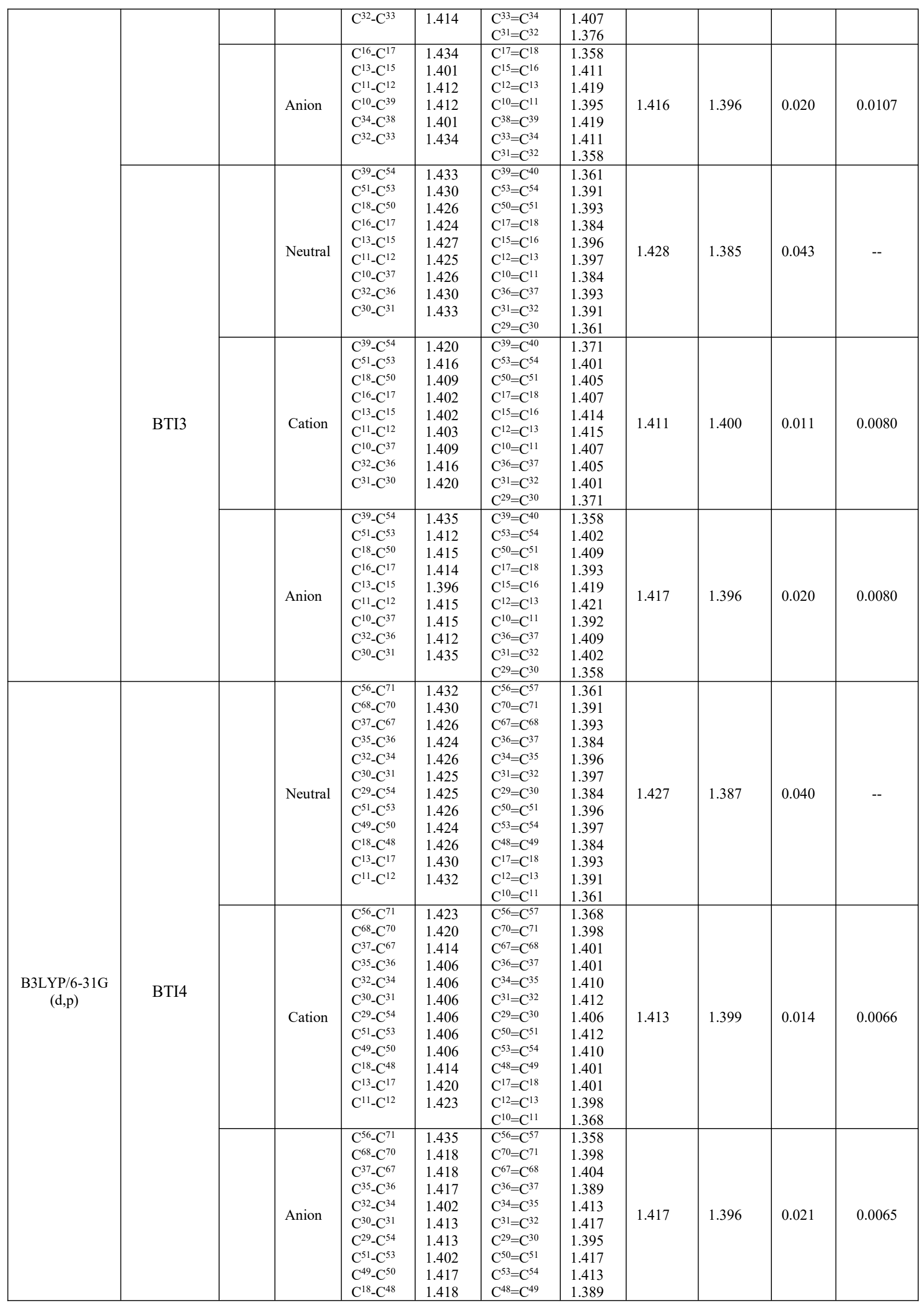




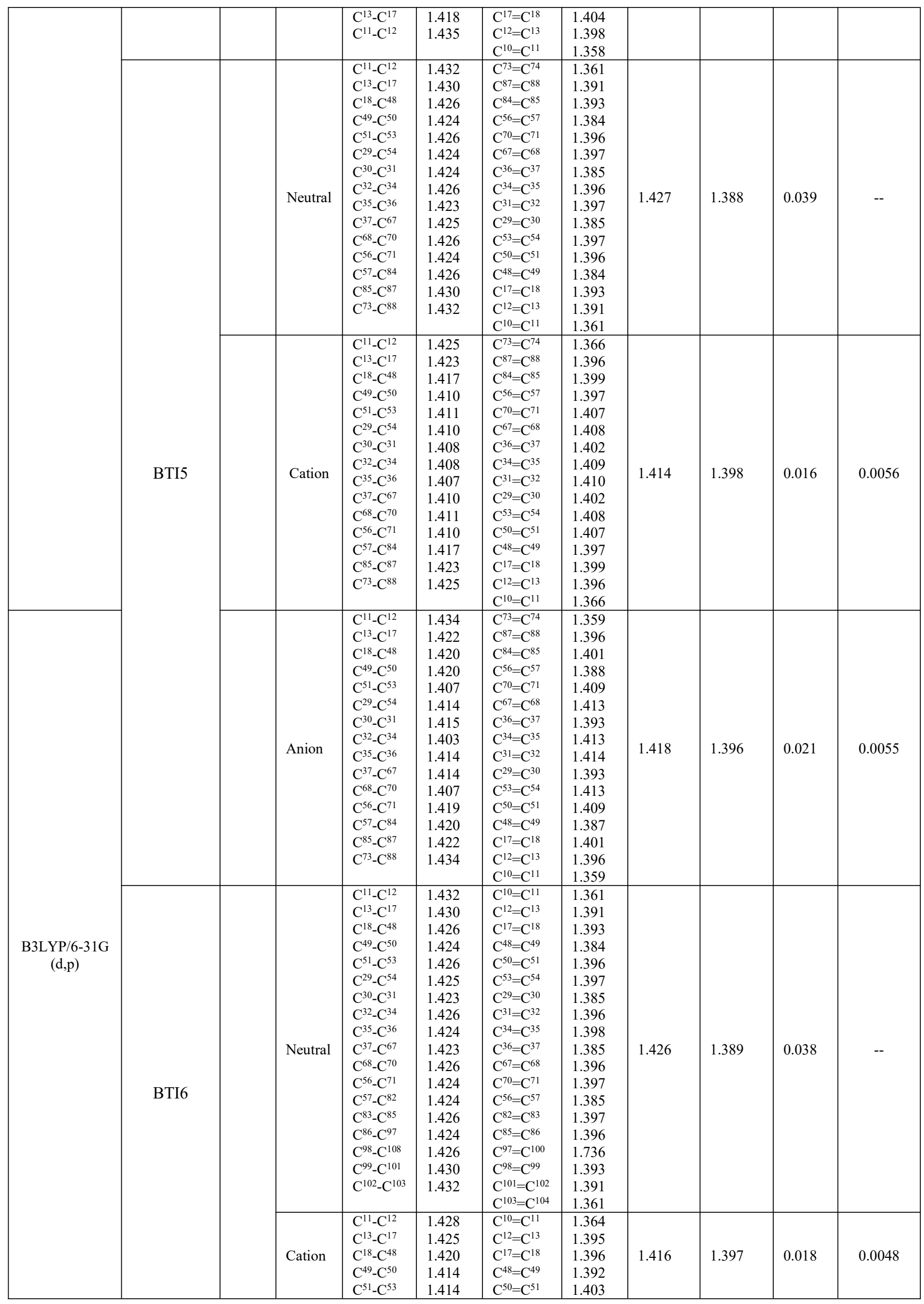




\begin{tabular}{|c|c|c|c|c|c|c|c|c|c|c|}
\hline & & & $\begin{array}{l}C^{29}-C^{54} \\
C^{30}-C^{31} \\
C^{32}-C^{34} \\
C^{35}-C^{36} \\
C^{37}-C^{67} \\
C^{68}-C^{70} \\
C^{56}-C^{71} \\
C^{57}-C^{82} \\
C^{83}-C^{85} \\
C^{86}-C^{97} \\
C^{98}-C^{108} \\
C^{99}-C^{101} \\
C^{102}-C^{103}\end{array}$ & $\begin{array}{l}1.413 \\
1.409 \\
1.409 \\
1.408 \\
1.408 \\
1.409 \\
1.409 \\
1.413 \\
1.414 \\
1.414 \\
1.420 \\
1.425 \\
1.428\end{array}$ & $\begin{array}{l}\mathrm{C}^{53}=\mathrm{C}^{54} \\
\mathrm{C}^{29}=\mathrm{C}^{30} \\
\mathrm{C}^{31}=\mathrm{C}^{32} \\
\mathrm{C}^{34}=\mathrm{C}^{35} \\
\mathrm{C}^{36}=\mathrm{C}^{37} \\
\mathrm{C}^{67}=\mathrm{C}^{68} \\
\mathrm{C}^{70}=\mathrm{C}^{71} \\
\mathrm{C}^{56}=\mathrm{C}^{57} \\
\mathrm{C}^{82}=\mathrm{C}^{83} \\
\mathrm{C}^{85}=\mathrm{C}^{86} \\
\mathrm{C}^{97}=\mathrm{C}^{100} \\
\mathrm{C}^{98}=\mathrm{C}^{99} \\
\mathrm{C}^{101}=\mathrm{C}^{102} \\
\mathrm{C}^{103}=\mathrm{C}^{104}\end{array}$ & $\begin{array}{l}1.405 \\
1.399 \\
1.408 \\
1.409 \\
1.402 \\
1.409 \\
1.408 \\
1.399 \\
1.405 \\
1.403 \\
1.392 \\
1.396 \\
1.395 \\
1.364\end{array}$ & & & & \\
\hline \multirow{3}{*}{$\begin{array}{c}\text { B3LYP/6-31G } \\
(\mathrm{d}, \mathrm{p})\end{array}$} & & Anion & $\begin{array}{l}C^{11}-C^{12} \\
C^{13}-C^{17} \\
C^{18}-C^{48} \\
C^{49}-C^{50} \\
C^{51}-C^{53} \\
C^{29}-C^{54} \\
C^{30}-C^{31} \\
C^{32}-C^{34} \\
C^{35}-C^{36} \\
C^{37}-C^{67} \\
C^{68}-C^{70} \\
C^{56}-C^{71} \\
C^{57}-C^{82} \\
C^{83}-C^{85} \\
C^{86}-C^{97} \\
C^{98}-C^{108} \\
C^{99}-C^{101} \\
C^{102}-C^{103}\end{array}$ & $\begin{array}{l}1.434 \\
1.424 \\
1.422 \\
1.421 \\
1.411 \\
1.416 \\
1.416 \\
1.406 \\
1.415 \\
1.414 \\
1.406 \\
1.417 \\
1.416 \\
1.412 \\
1.421 \\
1.422 \\
1.424 \\
1.434\end{array}$ & $\begin{array}{l}C^{10}=C^{11} \\
C^{12}=C^{13} \\
C^{17}=C^{18} \\
C^{48}=C^{49} \\
C^{50}=C^{51} \\
C^{53}=C^{54} \\
C^{29}=C^{30} \\
C^{31}=C^{32} \\
C^{34}=C^{35} \\
C^{36}=C^{37} \\
C^{67}=C^{68} \\
C^{70}=C^{71} \\
C^{56}=C^{57} \\
C^{82}=C^{83} \\
C^{85}=C^{86} \\
C^{97}=C^{100} \\
C^{98}=C^{99} \\
C^{101}=C^{102} \\
C^{103}=C^{104}\end{array}$ & $\begin{array}{l}1.359 \\
1.394 \\
1.399 \\
1.386 \\
1.406 \\
1.409 \\
1.391 \\
1.410 \\
1.412 \\
1.393 \\
1.411 \\
1.411 \\
1.391 \\
1.409 \\
1.406 \\
1.386 \\
1.399 \\
1.394 \\
1.359 \\
\end{array}$ & 1.418 & 1.396 & 0.022 & 0.0048 \\
\hline & \multirow[t]{2}{*}{ BTI7 } & Neutral & $\begin{array}{l}C^{11}-C^{12} \\
C^{13}-C^{17} \\
C^{18}-C^{48} \\
C^{49}-C^{50} \\
C^{51}-C^{53} \\
C^{29}-C^{54} \\
C^{30}-C^{31} \\
C^{32}-C^{34} \\
C^{35}-C^{36} \\
C^{37}-C^{67} \\
C^{68}-C^{70} \\
C^{56}-C^{71} \\
C^{57}-C^{82} \\
C^{83}-C^{85} \\
C^{86}-C^{97} \\
C^{98}-C^{106} \\
C^{99}-C^{101} \\
C^{102}-C^{103} \\
C^{104}-C^{124} \\
C^{119}-C^{123} \\
C^{117}-C^{118}\end{array}$ & $\begin{array}{l}1.432 \\
1.430 \\
1.426 \\
1.424 \\
1.426 \\
1.425 \\
1.423 \\
1.426 \\
1.424 \\
1.424 \\
1.426 \\
1.423 \\
1.423 \\
1.426 \\
1.424 \\
1.424 \\
1.426 \\
1.424 \\
1.426 \\
1.430 \\
1.432\end{array}$ & $\begin{array}{l}\mathrm{C}^{10}=\mathrm{C}^{11} \\
\mathrm{C}^{12}=\mathrm{C}^{13} \\
\mathrm{C}^{17}=\mathrm{C}^{18} \\
\mathrm{C}^{48}=\mathrm{C}^{49} \\
\mathrm{C}^{50}=\mathrm{C}^{51} \\
\mathrm{C}^{53}=\mathrm{C}^{54} \\
\mathrm{C}^{29}=\mathrm{C}^{30} \\
\mathrm{C}^{31}=\mathrm{C}^{32} \\
\mathrm{C}^{34}=\mathrm{C}^{35} \\
\mathrm{C}^{36}=\mathrm{C}^{37} \\
\mathrm{C}^{67}=\mathrm{C}^{68} \\
\mathrm{C}^{70}=\mathrm{C}^{71} \\
\mathrm{C}^{56}=\mathrm{C}^{57} \\
\mathrm{C}^{82}=\mathrm{C}^{83} \\
\mathrm{C}^{85}=\mathrm{C}^{86} \\
\mathrm{C}^{97}=\mathrm{C}^{106} \\
\mathrm{C}^{98}=\mathrm{C}^{99} \\
\mathrm{C}^{101}=\mathrm{C}^{102} \\
\mathrm{C}^{103}=\mathrm{C}^{104} \\
\mathrm{C}^{123}-C^{124} \\
\mathrm{C}^{118}-\mathrm{C}^{119} \\
\mathrm{C}^{116}-\mathrm{C}^{117}\end{array}$ & \begin{tabular}{l|l|}
1.361 \\
1.391 \\
1.393 \\
1.384 \\
1.396 \\
1.397 \\
1.385 \\
1.396 \\
1.397 \\
1.385 \\
1.397 \\
1.396 \\
1.385 \\
1.396 \\
1.397 \\
1.385 \\
1.397 \\
1.396 \\
1.384 \\
1.393 \\
1.391 \\
1.361 \\
\end{tabular} & 1.426 & 1.389 & 0.037 & -- \\
\hline & & Cation & $\begin{array}{l}C^{11}-C^{12} \\
C^{13}-C^{17} \\
C^{18}-C^{48} \\
C^{49}-C^{50} \\
C^{51}-C^{53} \\
C^{29}-C^{54} \\
C^{30}-C^{31} \\
C^{32}-C^{34} \\
C^{35}-C^{36} \\
C^{37}-C^{67} \\
C^{68}-C^{70} \\
C^{56}-C^{71} \\
C^{57}-C^{82} \\
C^{83}-C^{85} \\
C^{86}-C^{97} \\
C^{98}-C^{106} \\
C^{99}-C^{101} \\
C^{102}-C^{103} \\
C^{104}-C^{124} \\
C^{119}-C^{123}\end{array}$ & \begin{tabular}{l|}
1.429 \\
1.426 \\
1.421 \\
1.416 \\
1.417 \\
1.416 \\
1.411 \\
1.412 \\
1.411 \\
1.409 \\
1.409 \\
1.409 \\
1.411 \\
1.412 \\
1.411 \\
1.416 \\
1.417 \\
1.416 \\
1.421 \\
1.426
\end{tabular} & $\begin{array}{l}C^{10}=C^{11} \\
C^{12}=C^{13} \\
C^{17}=C^{18} \\
C^{48}=C^{49} \\
C^{50}=C^{51} \\
C^{53}=C^{54} \\
C^{29}=C^{30} \\
C^{31}=C^{32} \\
C^{34}=C^{35} \\
C^{36}=C^{37} \\
C^{67}=C^{68} \\
C^{70}=C^{71} \\
C^{56}=C^{57} \\
C^{82}=C^{83} \\
C^{85}=C^{86} \\
C^{97}=C^{106} \\
C^{98}=C^{99} \\
C^{101}=C^{102} \\
C^{103}=C^{104} \\
C^{123}-C^{124}\end{array}$ & $\begin{array}{l}1.364 \\
1.394 \\
1.395 \\
1.390 \\
1.402 \\
1.403 \\
1.396 \\
1.406 \\
1.407 \\
1.400 \\
1.408 \\
1.408 \\
1.400 \\
1.407 \\
1.406 \\
1.396 \\
1.403 \\
1.402 \\
1.390 \\
1.395\end{array}$ & 1.416 & 1.397 & 0.020 & 0.0043 \\
\hline
\end{tabular}




\begin{tabular}{|c|c|c|c|c|c|c|c|c|c|c|}
\hline & & & $\mathrm{C}^{117}-\mathrm{C}^{118}$ & 1.429 & $\begin{array}{l}C^{118}-C^{119} \\
C^{116}-C^{117}\end{array}$ & $\begin{array}{l}1.394 \\
1.364\end{array}$ & & & & \\
\hline \multirow{4}{*}{$\begin{array}{l}\text { B3LYP/6-31G } \\
(\mathrm{d}, \mathrm{p})\end{array}$} & & Anion & $\begin{array}{l}C^{11}-C^{12} \\
C^{13}-C^{17} \\
C^{18}-C^{48} \\
C^{49}-C^{50} \\
C^{51}-C^{53} \\
C^{29}-C^{54} \\
C^{30}-C^{31} \\
C^{32}-C^{34} \\
C^{35}-C^{36} \\
C^{37}-C^{67} \\
C^{68}-C^{70} \\
C^{56}-C^{71} \\
C^{57}-C^{82} \\
C^{83}-C^{85} \\
C^{86}-C^{97} \\
C^{98}-C^{106} \\
C^{99}-C^{101} \\
C^{102}-C^{103} \\
C^{104}-C^{124} \\
C^{119}-C^{123} \\
C^{117}-C^{118}\end{array}$ & $\begin{array}{l}1.434 \\
1.425 \\
1.423 \\
1.422 \\
1.414 \\
1.418 \\
1.418 \\
1.410 \\
1.416 \\
1.417 \\
1.408 \\
1.416 \\
1.415 \\
1.409 \\
1.419 \\
1.418 \\
1.414 \\
1.422 \\
1.423 \\
1.425 \\
1.434\end{array}$ & $\begin{array}{l}\mathrm{C}^{10}=\mathrm{C}^{11} \\
\mathrm{C}^{12}=\mathrm{C}^{13} \\
\mathrm{C}^{17}=\mathrm{C}^{18} \\
\mathrm{C}^{48}=\mathrm{C}^{49} \\
\mathrm{C}^{50}=\mathrm{C}^{51} \\
\mathrm{C}^{53}=\mathrm{C}^{54} \\
\mathrm{C}^{29}=\mathrm{C}^{30} \\
\mathrm{C}^{31}=\mathrm{C}^{32} \\
\mathrm{C}^{34}=\mathrm{C}^{35} \\
\mathrm{C}^{36}=\mathrm{C}^{37} \\
\mathrm{C}^{67}=\mathrm{C}^{68} \\
\mathrm{C}^{70}=\mathrm{C}^{71} \\
\mathrm{C}^{56}=\mathrm{C}^{57} \\
\mathrm{C}^{82}=\mathrm{C}^{83} \\
\mathrm{C}^{85}=\mathrm{C}^{86} \\
\mathrm{C}^{97}=\mathrm{C}^{106} \\
\mathrm{C}^{98}=\mathrm{C}^{99} \\
\mathrm{C}^{101}=\mathrm{C}^{102} \\
\mathrm{C}^{103}=\mathrm{C}^{104} \\
\mathrm{C}^{123}-\mathrm{C}^{124} \\
\mathrm{C}^{118}-\mathrm{C}^{119} \\
\mathrm{C}^{116}-\mathrm{C}^{117}\end{array}$ & $\begin{array}{l}1.359 \\
1.393 \\
1.398 \\
1.386 \\
1.404 \\
1.407 \\
1.390 \\
1.407 \\
1.410 \\
1.391 \\
1.410 \\
1.409 \\
1.392 \\
1.409 \\
1.409 \\
1.390 \\
1.407 \\
1.404 \\
1.386 \\
1.398 \\
1.359 \\
1.393 \\
\end{array}$ & 1.419 & 1.396 & 0.023 & 0.0042 \\
\hline & \multirow{3}{*}{ BTI8 } & Neutral & $\begin{array}{l}C^{11}-C^{12} \\
C^{13}-C^{17} \\
C^{18}-C^{48} \\
C^{49}-C^{50} \\
C^{51}-C^{53} \\
C^{29}-C^{54} \\
C^{30}-C^{31} \\
C^{32}-C^{34} \\
C^{35}-C^{36} \\
C^{37}-C^{67} \\
C^{68}-C^{70} \\
C^{56}-C^{71} \\
C^{57}-C^{82} \\
C^{83}-C^{85} \\
C^{86}-C^{97} \\
C^{98}-C^{106} \\
C^{99}-C^{101} \\
C^{102}-C^{103} \\
C^{104}-C^{122} \\
C^{119}-C^{121} \\
C^{117}-C^{118} \\
C^{116}-C^{141} \\
C^{136}-C^{140} \\
C^{134}-C^{135}\end{array}$ & $\begin{array}{l}1.432 \\
1.430 \\
1.426 \\
1.424 \\
1.426 \\
1.425 \\
1.423 \\
1.426 \\
1.424 \\
1.424 \\
1.426 \\
1.423 \\
1.423 \\
1.426 \\
1.424 \\
1.423 \\
1.426 \\
1.424 \\
1.424 \\
1.426 \\
1.424 \\
1.426 \\
1.430 \\
1.432\end{array}$ & $\begin{array}{l}\mathrm{C}^{10}=\mathrm{C}^{11} \\
\mathrm{C}^{12}=\mathrm{C}^{13} \\
\mathrm{C}^{17}=\mathrm{C}^{18} \\
\mathrm{C}^{48}=\mathrm{C}^{49} \\
\mathrm{C}^{50}=\mathrm{C}^{51} \\
\mathrm{C}^{53}=\mathrm{C}^{54} \\
\mathrm{C}^{29}=\mathrm{C}^{30} \\
\mathrm{C}^{31}=\mathrm{C}^{32} \\
\mathrm{C}^{34}=\mathrm{C}^{35} \\
\mathrm{C}^{36}=\mathrm{C}^{37} \\
\mathrm{C}^{67}=\mathrm{C}^{68} \\
\mathrm{C}^{70}=\mathrm{C}^{71} \\
\mathrm{C}^{56}=\mathrm{C}^{57} \\
\mathrm{C}^{82}=\mathrm{C}^{83} \\
\mathrm{C}^{85}=\mathrm{C}^{86} \\
\mathrm{C}^{97}=\mathrm{C}^{106} \\
\mathrm{C}^{98}=\mathrm{C}^{99} \\
\mathrm{C}^{101}=\mathrm{C}^{102} \\
\mathrm{C}^{103}=\mathrm{C}^{104} \\
\mathrm{C}^{118}-\mathrm{C}^{119} \\
\mathrm{C}^{121}-\mathrm{C}^{122} \\
\mathrm{C}^{116}-\mathrm{C}^{117} \\
\mathrm{C}^{140}-\mathrm{C}^{141} \\
\mathrm{C}^{133}-\mathrm{C}^{134} \\
\mathrm{C}^{135}-\mathrm{C}^{136}\end{array}$ & $\begin{array}{l}1.361 \\
1.391 \\
1.393 \\
1.384 \\
1.396 \\
1.397 \\
1.385 \\
1.396 \\
1.397 \\
1.385 \\
1.397 \\
1.396 \\
1.385 \\
1.396 \\
1.398 \\
1.385 \\
1.396 \\
1.397 \\
1.385 \\
1.396 \\
1.397 \\
1.384 \\
1.393 \\
1.361 \\
1.391 \\
\end{array}$ & 1.426 & 1.390 & 0.036 & -- \\
\hline & & Cation & $\begin{array}{l}C^{11}-C^{12} \\
C^{13}-C^{17} \\
C^{18}-C^{48} \\
C^{49}-C^{50} \\
C^{51}-C^{53} \\
C^{29}-C^{54} \\
C^{30}-C^{31} \\
C^{32}-C^{34} \\
C^{35}-C^{36} \\
C^{37}-C^{67} \\
C^{68}-C^{70} \\
C^{56}-C^{71} \\
C^{57}-C^{82} \\
C^{83}-C^{85} \\
C^{86}-C^{97} \\
C^{98}-C^{106} \\
C^{99}-C^{101} \\
C^{102}-C^{103} \\
C^{104}-C^{122} \\
C^{119}-C^{121} \\
C^{117}-C^{118} \\
C^{116}-C^{141} \\
C^{136}-C^{140} \\
C^{134}-C^{135}\end{array}$ & $\begin{array}{l}1.430 \\
1.427 \\
1.422 \\
1.418 \\
1.419 \\
1.418 \\
1.414 \\
1.414 \\
1.414 \\
1.411 \\
1.411 \\
1.411 \\
1.411 \\
1.411 \\
1.411 \\
1.414 \\
1.414 \\
1.414 \\
1.418 \\
1.419 \\
1.418 \\
1.422 \\
1.427 \\
1.430\end{array}$ & $\begin{array}{l}\mathrm{C}^{10}=\mathrm{C}^{11} \\
\mathrm{C}^{12}=\mathrm{C}^{13} \\
\mathrm{C}^{17}=\mathrm{C}^{18} \\
\mathrm{C}^{48}=\mathrm{C}^{49} \\
\mathrm{C}^{50}=\mathrm{C}^{51} \\
\mathrm{C}^{53}=\mathrm{C}^{54} \\
\mathrm{C}^{29}=\mathrm{C}^{30} \\
\mathrm{C}^{31}=\mathrm{C}^{32} \\
\mathrm{C}^{34}=\mathrm{C}^{35} \\
\mathrm{C}^{36}=\mathrm{C}^{37} \\
\mathrm{C}^{67}=\mathrm{C}^{68} \\
\mathrm{C}^{70}=\mathrm{C}^{71} \\
\mathrm{C}^{56}=\mathrm{C}^{57} \\
\mathrm{C}^{82}=\mathrm{C}^{83} \\
\mathrm{C}^{85}=\mathrm{C}^{86} \\
\mathrm{C}^{97}=\mathrm{C}^{106} \\
\mathrm{C}^{98}=\mathrm{C}^{99} \\
\mathrm{C}^{101}=\mathrm{C}^{102} \\
\mathrm{C}^{103}=\mathrm{C}^{104} \\
\mathrm{C}^{118}-\mathrm{C}^{119} \\
\mathrm{C}^{121}-\mathrm{C}^{122} \\
\mathrm{C}^{116}-\mathrm{C}^{117} \\
\mathrm{C}^{140}-\mathrm{C}^{141} \\
\mathrm{C}^{133}-\mathrm{C}^{134} \\
\mathrm{C}^{135}-\mathrm{C}^{136}\end{array}$ & $\begin{array}{l}1.363 \\
1.394 \\
1.395 \\
1.389 \\
1.401 \\
1.401 \\
1.394 \\
1.404 \\
1.405 \\
1.398 \\
1.406 \\
1.407 \\
1.399 \\
1.407 \\
1.406 \\
1.398 \\
1.405 \\
1.404 \\
1.394 \\
1.401 \\
1.401 \\
1.389 \\
1.395 \\
1.363 \\
1.394 \\
\end{array}$ & 1.417 & 1.396 & 0.021 & 0.0039 \\
\hline & & Anion & $\mathrm{C}^{11}-\mathrm{C}^{12}$ & 1.434 & $\mathrm{C}^{10}=\mathrm{C}^{11}$ & 1.359 & 1.419 & 1.396 & 0.024 & 0.0037 \\
\hline
\end{tabular}




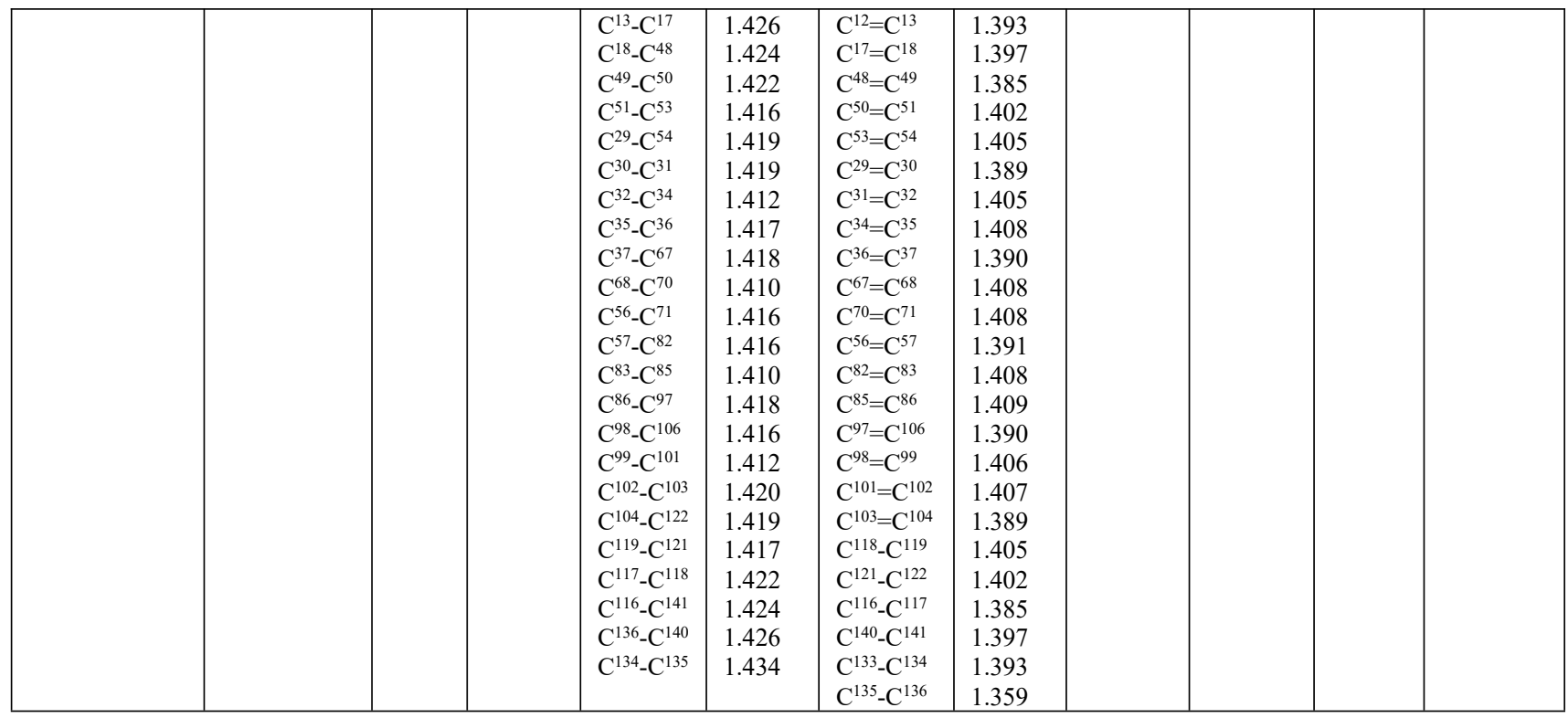

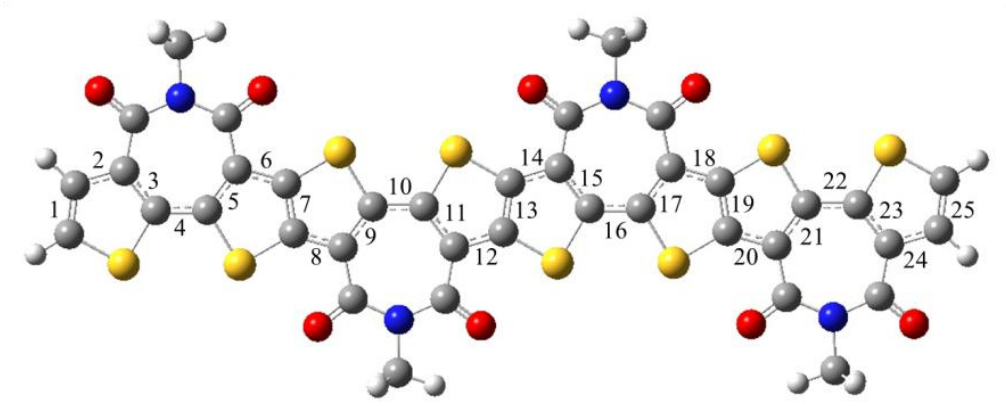

Figure S2. Atomic labeling in the BTI series of oligomers.

BTI7

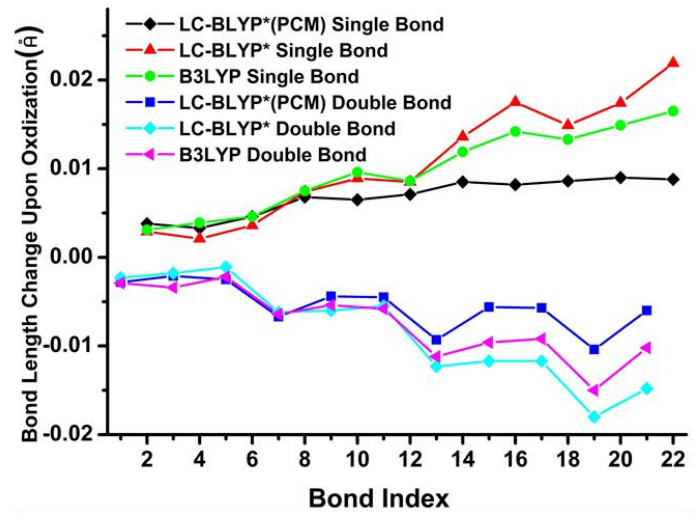

BTI8

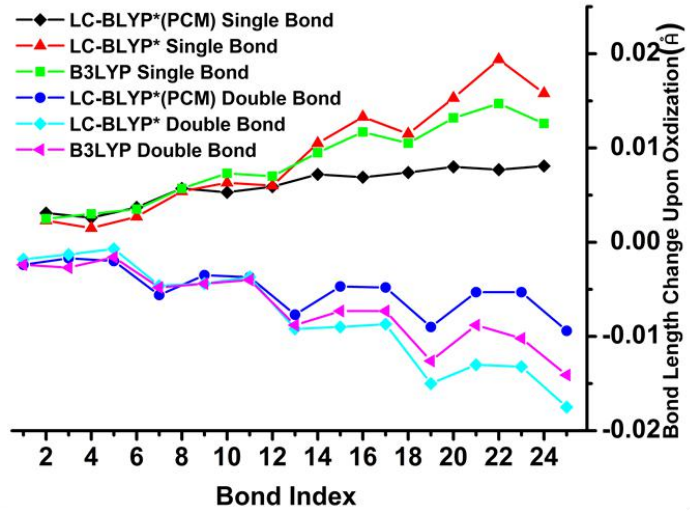

Figure S3. Changes in bond length in the BTI7 and BTI8 oligomers upon oxidation calculated at the LC-BLYP*(PCM)/6-31G(d), LC-BLYP*/6-31G(d) and B3LYP/6-31G(d) levels. Bond indices correspond to the $\mathrm{C}-\mathrm{C}$ single and double bonds starting from one (end) towards the center, see Figure S2. 
(a)

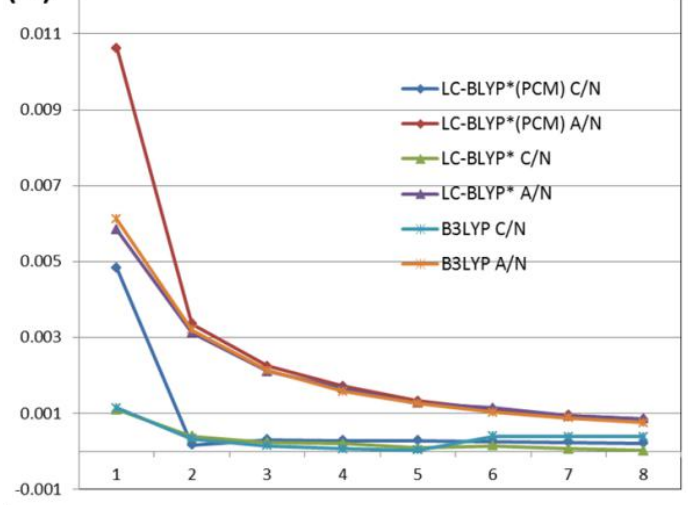

(b)

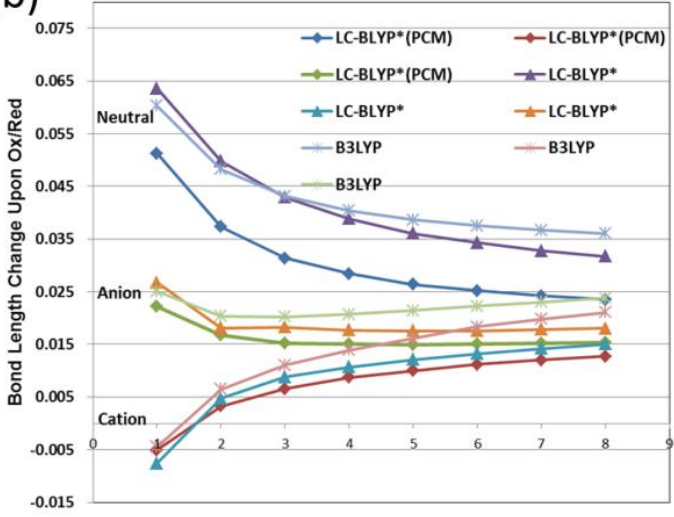

Figure S4. The evolution of the total bond length of cations/anions relative to neutral states (a), and the evolution of bond length alternation(BLA) for cations/anions/ neutral (b) of BTI-BTI8 calculated at the LC-BLYP*(PCM)/6-31G(d), LC-BLYP*/6-31G(d,p) and B3LYP/6-31G $(d, p)$ levels. Bond lengths in Angstroms. 
Table S3. The frontier orbitals energies (HOMO and LUMO), HOMO-LUMO gaps, optical gaps, the vertical ionization potentials $\left(\mathrm{IP}_{\mathrm{v}}\right)$ and electron affinity energies $\left(\mathrm{EA}_{\mathrm{v}}\right)$, hole and electron reorganization energies $\left(\lambda_{+}\right.$and $\left.\lambda_{-}\right)$and hole and electron-transfer mobilities $\left(\mu_{+}\right.$and $\left.\mu_{-}\right)$of BTIBTI8 calculated with LC-BLYP*(PCM, solid), LC-BLYP*(chlorobenzene), LC-BLYP*, and B3LYP methods with 6-31G(d,p) basis set. All energies in $\mathrm{eV}$ and mobilities in $\mathrm{cm}^{2} \mathrm{~V}^{-1} \mathrm{~S}^{-1}$.

\begin{tabular}{|c|c|c|c|c|c|c|c|c|c|c|c|}
\hline Mole & $\varepsilon$ & $\omega$ & HOMO & LUMO & $\begin{array}{l}\text { Optical gap } \\
\text { TD-DFT }\end{array}$ & $\mathrm{IP}_{\mathrm{v}}$ & $\mathrm{EA}_{\mathrm{v}}$ & $\lambda_{+}$ & $\lambda$ & $\mu_{+}$ & $\mu$ \\
\hline & \multicolumn{11}{|c|}{ LC-BLYP*(soild)/6-31G(d,p) } \\
\hline BTI & 3.61 & 0.066 & -6.16 & $\begin{array}{c}-1.73 \\
(-2.29)^{\mathrm{a}}\end{array}$ & $3.20(3.16)^{b}$ & 6.19 & 1.76 & 0.29 & 0.31 & 1.19 & \multirow[t]{2}{*}{0.69} \\
\hline BTI & $3.61^{\mathrm{c}}$ & $\begin{array}{c}0.14^{\mathrm{c}} \\
(\alpha=0.2769 \\
\beta=0.0001)\end{array}$ & $-6.28^{c}$ & $-1.90^{c}$ & $3.57^{\mathrm{c}}$ & $6.43^{c}$ & $1.76^{\mathrm{c}}$ & $0.40^{\mathrm{c}}$ & $0.42^{\mathrm{c}}$ & & \\
\hline BTI2 & 5.09 & 0.038 & -5.51 & $\begin{array}{c}-2.55 \\
(-2.90)^{\mathrm{a}}\end{array}$ & $2.35(2.48)^{b}$ & 5.53 & 2.57 & 0.20 & 0.17 & 1.98 & $\begin{array}{c}6.34 \mathrm{E}- \\
02 \\
(0.045)^{\mathrm{d}}\end{array}$ \\
\hline BTI2 & $5.09^{c}$ & $\begin{array}{c}0.1622^{c} \\
(\alpha=0.1964, \\
\beta=0.0001)\end{array}$ & $-5.63^{c}$ & $-2.65^{c}$ & $2.67^{\mathrm{c}}$ & $5.77^{\mathrm{c}}$ & $2.53^{\mathrm{c}}$ & $0.28^{\mathrm{c}}$ & $0.26^{\mathrm{c}}$ & & \\
\hline ВТI3 & 7.41 & 0.025 & -5.21 & $\begin{array}{c}-2.95 \\
(-3.15)^{\mathrm{a}}\end{array}$ & $1.91(2.19)^{\mathrm{b}}$ & 5.23 & 2.96 & 0.16 & 0.14 & 3.66 & $\begin{array}{c}1.29 \\
(0.013)^{\mathrm{d}}\end{array}$ \\
\hline ВTI3 & $7.41^{\mathrm{c}}$ & $\begin{array}{c}0.3310^{\mathrm{c}} \\
(\alpha=0.1349, \\
\beta=0.0001)\end{array}$ & $-5.28^{c}$ & $-3.04^{c}$ & $2.16^{\mathrm{c}}$ & $5.43^{\mathrm{c}}$ & $2.92^{\mathrm{c}}$ & $0.21^{\mathrm{c}}$ & $0.19^{c}$ & & \\
\hline BTI4 & 11.10 & 0.018 & -5.04 & $\begin{array}{c}-3.18 \\
(-3.26)^{\mathrm{a}}\end{array}$ & $1.66(2.00)^{b}$ & 5.05 & 3.18 & 0.13 & 0.11 & 6.93 & $\begin{array}{c}1.90 \\
(0.026)^{d}\end{array}$ \\
\hline BTI5 & 17.23 & 0.013 & -4.95 & $\begin{array}{c}-3.32 \\
(-3.55)^{\mathrm{a}}\end{array}$ & $1.50(1.93)^{\mathrm{b}}$ & 4.95 & 3.33 & 0.11 & 0.10 & $\begin{array}{c}9.70 \\
(0.0018)^{\mathrm{d}}\end{array}$ & $\begin{array}{c}0.46 \\
(0.013)^{d}\end{array}$ \\
\hline BTI6 & 29.28 & 0.010 & -4.88 & -3.42 & 1.41 & 4.88 & 3.42 & 0.09 & 0.09 & 12.55 & 1.51 \\
\hline BTI7 & 64.67 & 0.008 & -4.83 & -3.48 & 1.36 & 4.83 & 3.48 & 0.08 & 0.08 & 13.48 & 1.84 \\
\hline BTI8 & 498.00 & 0.006 & -4.80 & -3.53 & 1.31 & 4.80 & 3.53 & 0.07 & 0.07 & 16.60 & 3.02 \\
\hline & \multicolumn{11}{|c|}{ LC-BLYP*/6-31G(d,p) } \\
\hline BTI & -- & 0.235 & -7.92 & -0.48 & $3.69(3.19)^{\mathrm{e}}$ & 7.94 & 0.51 & 0.44 & 0.42 & 0.30 & 0.29 \\
\hline BTI2 & -- & 0.186 & -7.11 & -1.30 & $2.88(2.63)^{\mathrm{e}}$ & 7.13 & 1.30 & 0.36 & 0.30 & 0.46 & 0.02 \\
\hline BTI3 & -- & 0.159 & -6.72 & -1.73 & $2.44(2.31)^{\mathrm{e}}$ & 6.74 & 1.73 & 0.32 & 0.27 & 0.71 & 0.32 \\
\hline BTI4 & -- & 0.143 & -6.49 & -1.98 & $2.21(2.13)^{\mathrm{e}}$ & 6.52 & 1.98 & 0.29 & 0.25 & 1.27 & 0.40 \\
\hline BTI5 & -- & 0.133 & -6.35 & -2.14 & $2.05(2.04)^{\mathrm{e}}$ & 6.38 & 2.14 & 0.26 & 0.23 & 1.86 & 0.08 \\
\hline BTI6 & -- & 0.125 & -6.25 & -2.26 & 1.96 & 6.28 & 2.25 & 0.24 & 0.21 & 2.23 & 0.30 \\
\hline BTI7 & -- & 0.120 & -6.18 & -2.34 & 1.89 & 6.21 & 2.33 & 0.23 & 0.20 & 2.50 & 0.37 \\
\hline BTI8 & -- & 0.116 & -6.13 & -2.40 & 1.83 & 6.16 & 2.39 & 0.21 & 0.19 & 3.06 & 0.57 \\
\hline & \multicolumn{11}{|c|}{ B3LYP/6-31G(d,p) } \\
\hline BTI & -- & -- & -6.19 & -2.31 & -- & 7.86 & 0.68 & 0.37 & 0.40 & 0.54 & 0.33 \\
\hline BTI2 & -- & -- & -5.86 & -2.83 & -- & 7.12 & 1.60 & 0.30 & 0.26 & 0.75 & 0.02 \\
\hline BTI3 & -- & -- & -5.71 & -3.09 & -- & 6.76 & 2.05 & 0.26 & 0.22 & 1.29 & 0.49 \\
\hline BTI4 & -- & -- & -5.65 & -3.23 & -- & 6.56 & 2.33 & 0.23 & 0.20 & 2.41 & 0.72 \\
\hline BTI5 & -- & -- & -5.61 & -3.32 & -- & 6.42 & 2.52 & 0.20 & 0.18 & 3.68 & 0.18 \\
\hline BTI6 & -- & -- & -5.58 & -3.38 & -- & 6.32 & 2.65 & 0.18 & 0.16 & 4.91 & 0.69 \\
\hline BTI7 & -- & -- & -5.57 & -3.43 & -- & 6.24 & 2.75 & 0.16 & 0.14 & 5.88 & 0.86 \\
\hline BTI8 & -- & -- & -5.56 & -3.46 & -- & 6.18 & 2.83 & 0.14 & 0.13 & 7.81 & 1.50 \\
\hline
\end{tabular}

${ }^{\mathrm{a}} \mathrm{LUMO}$ calculated from first reduction potential using $\mathrm{Fc} / \mathrm{Fc}+$ internal standard with $E_{\text {LUMO }}=-$ $\left(E_{r e}^{\text {onset }}+\left(E_{\frac{F c}{F c}+}\right)+4.8\right)$, refer 13 of the main text; ${ }^{\mathrm{b}}$ Derived from absorption of BTI-BTI5 films, refer 13 of the main text; ${ }^{c}$ Electronic structure of BTI series at LC-BLYP* $(\omega, \alpha, \varepsilon) / 6-31 \mathrm{G}(\mathrm{d}, \mathrm{p})$ level; ${ }^{\mathrm{d}}$ Derived from SI of refer 13; ${ }^{\mathrm{e}}$ Derived from absorption of BTI-BTI5 in chlorobenzene solution at $373 \mathrm{~K}$, refer 13 of the main text. 
Table S4. Calculated electronic structural properties of the BTI analogues at a B3LYP/6-31G(d,p) level.

\begin{tabular}{cccccccccc}
\hline Molecules & $\begin{array}{c}\text { HOMO } \\
{[\mathrm{eV}]}\end{array}$ & $\begin{array}{c}\text { LUMO } \\
{[\mathrm{eV}]}\end{array}$ & $\begin{array}{c}\text { gap } \\
{[\mathrm{eV}]}\end{array}$ & $\begin{array}{c}\mathrm{IP}_{\text {adia }} \\
{[\mathrm{eV}]}\end{array}$ & $\begin{array}{c}\mathrm{EA}_{\text {adia }} \\
{[\mathrm{eV}]}\end{array}$ & $\begin{array}{c}\mathrm{IP}_{\text {vert }} \\
{[\mathrm{eV}]}\end{array}$ & $\begin{array}{c}\mathrm{EA}_{\text {vert }} \\
{[\mathrm{eV}]}\end{array}$ & $\begin{array}{c}\lambda_{+} \\
{[\mathrm{eV}]}\end{array}$ & $\begin{array}{c}\boldsymbol{\lambda}_{-} \\
{[\mathrm{eV}]}\end{array}$ \\
\hline TBDI & -6.25 & -2.59 & 3.66 & 7.43 & -1.50 & 7.60 & -1.27 & 0.34 & 0.43 \\
BTI-2T & -5.33 & -2.65 & 2.68 & 6.30 & -1.62 & 6.43 & -1.50 & 0.27 & 0.24 \\
BTTT1 & -5.12 & -1.55 & 3.56 & 6.14 & -0.46 & 6.47 & -0.23 & 0.51 & 0.39 \\
BTI-T-T & -5.70 & -2.63 & 3.07 & 6.05 & -1.69 & 6.20 & -1.56 & 0.28 & 0.24 \\
\hline
\end{tabular}

HOMO
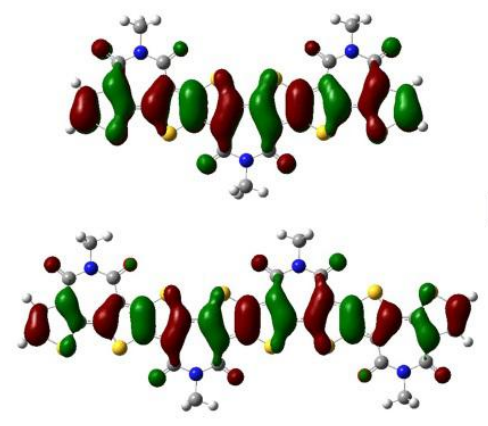

BTI4

BTI3

\section{LUMO}
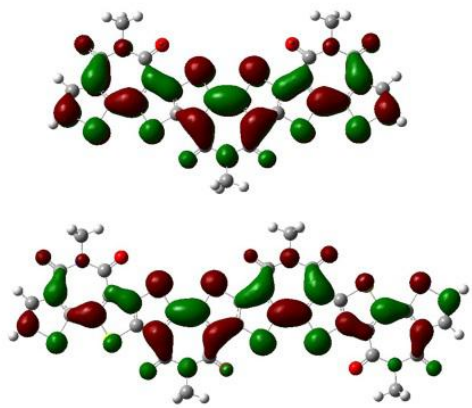

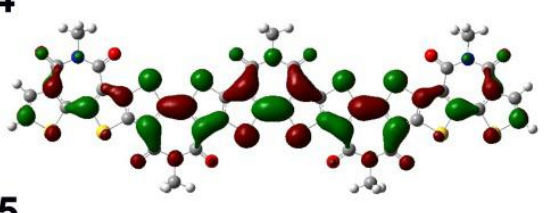

BTI5

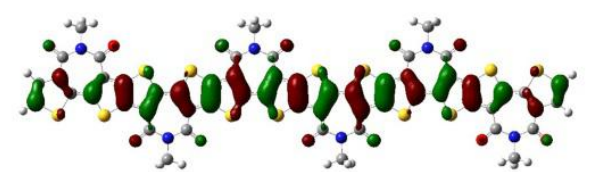

BTI6
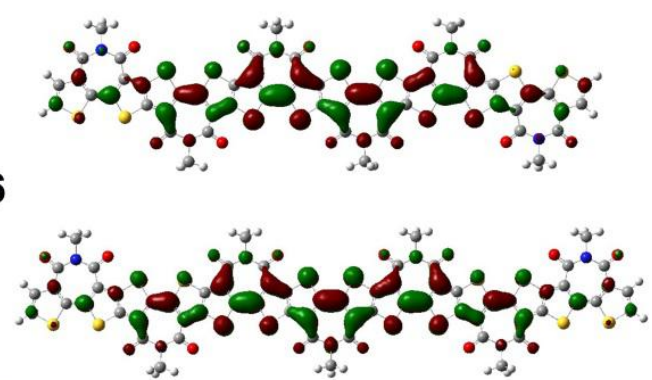

BTI7

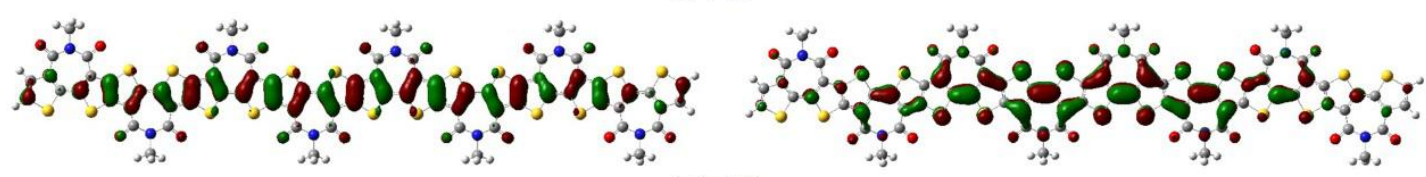

BTI8

Figure S5. Simulated maps of the frontier molecular orbitals of BTI3-BTI8 based on the optimized geometries at LC-BLYP*(PCM, solid)/6-31G(d,p) level. 
(a)

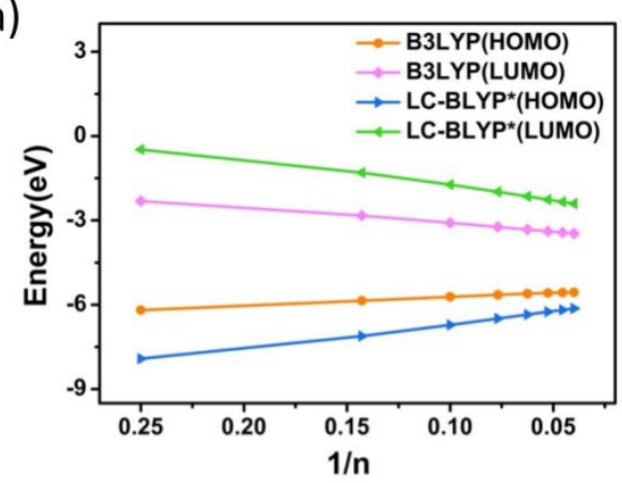

(c)

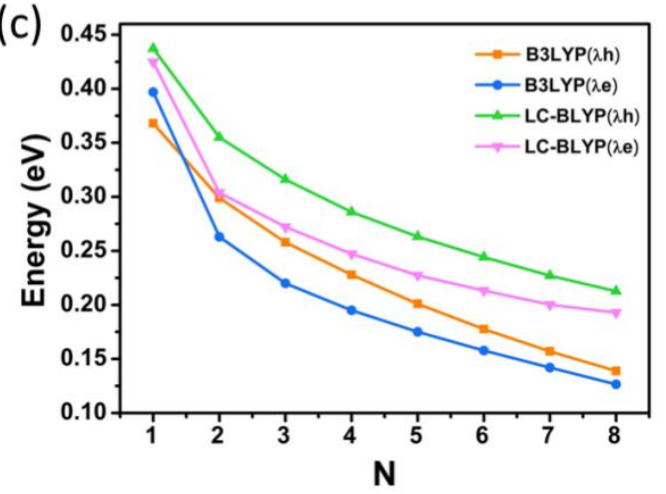

(b)

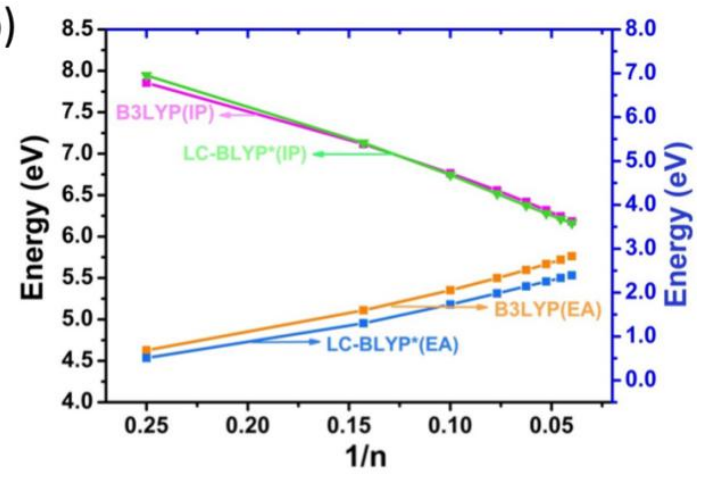

(d)

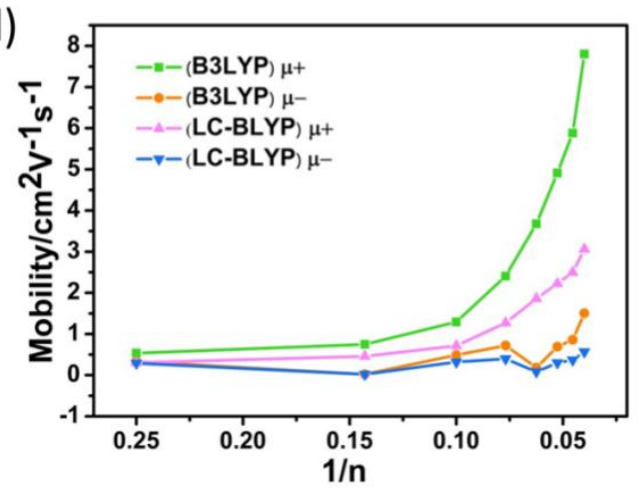

Figure S6. (a)The frontier molecular orbital energy level, (b) the vertical ionization potential and vertical electron affinity, (c) hole and electron reorganization energies, and (d) hole and electrontransfer mobilities changing with reciprocal of the double bond $(1 / \mathrm{n})$ or oligomer length of BTIBTI8 calculated at LC-BLYP*/6-31G(d,p) and B3LYP/6-31G(d,p) levels.

(a)

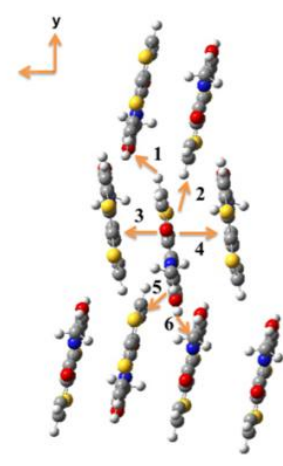

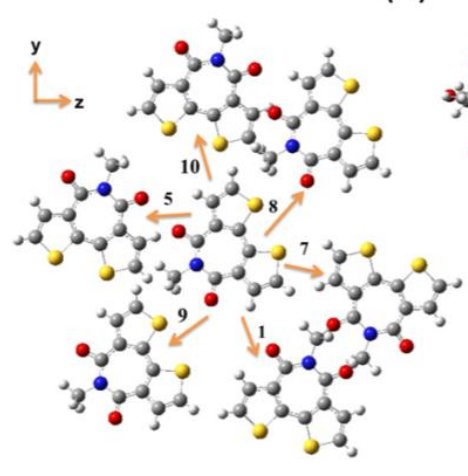

(b)

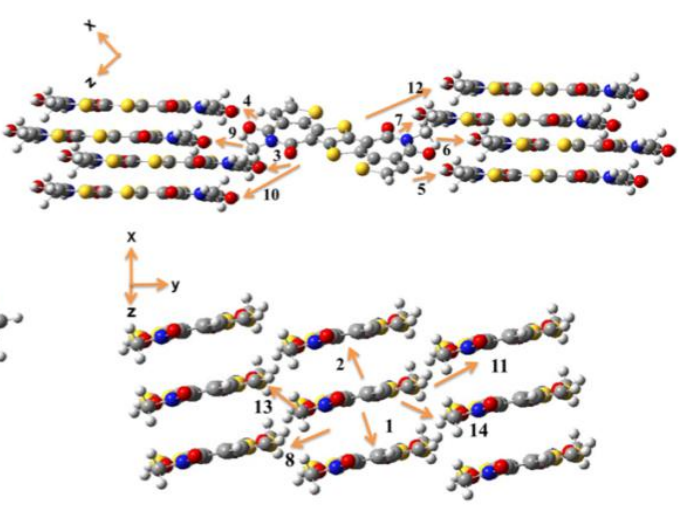

(c1)

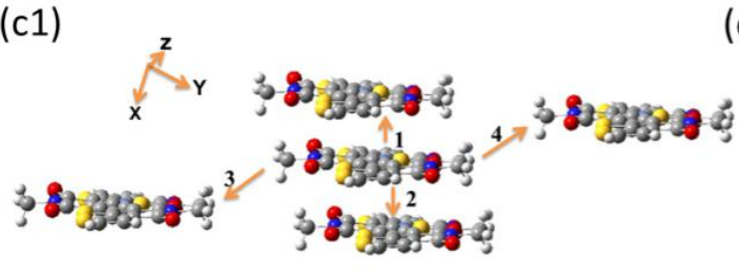

(c2)

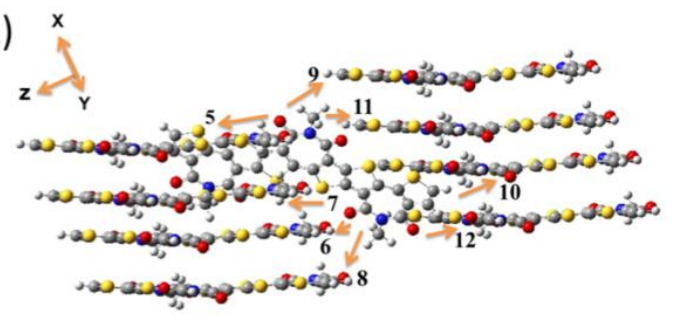


(d1)

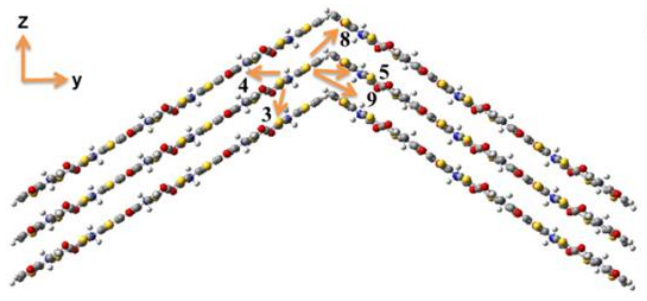

(e1)

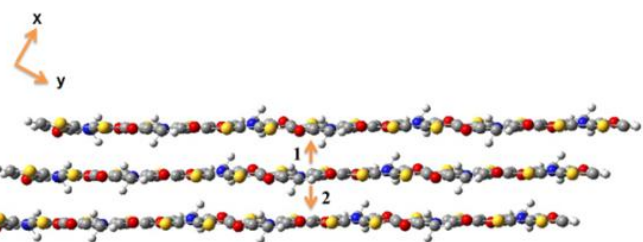

(f1)

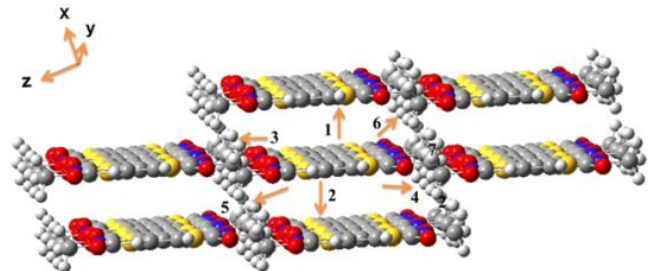

(d2)

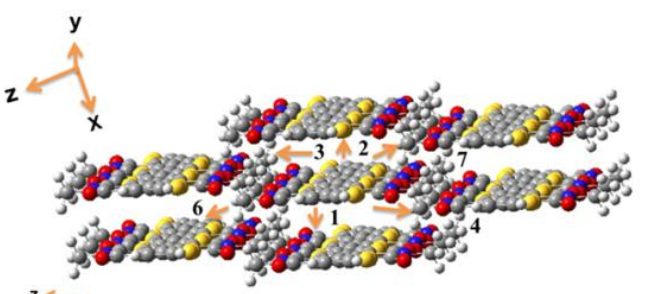

(e2)

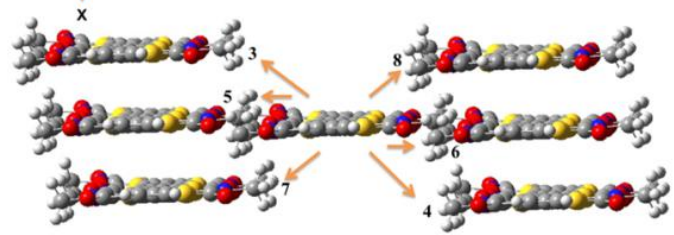

(f2)

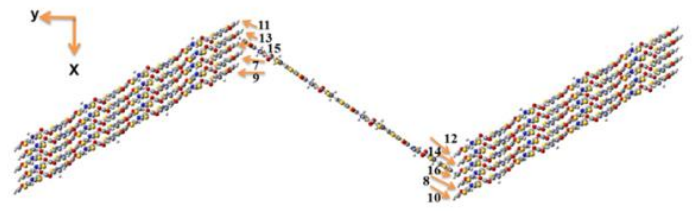

Figure S7. The main charge transport paths in the simulated crystals of BTI (a), BTI2 (b), BTI3 (c) BTI6 (d), BTI7 (e), BTI8 (f).

HOMO

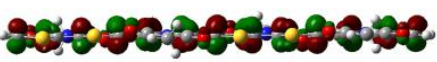

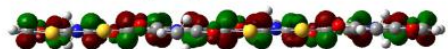

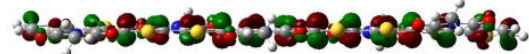

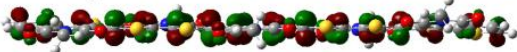

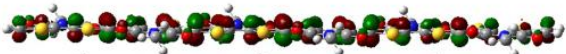
-

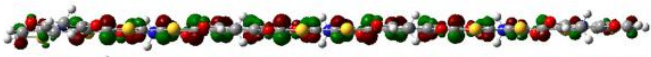
8ำ
BTI4

BTI5

BTI6

BTI7

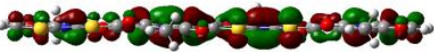

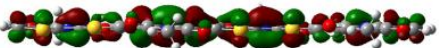

is

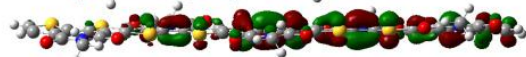

*

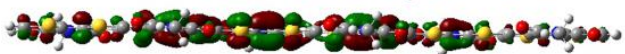

29050

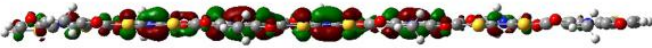

Figure S8. Frontier molecular orbitals (HOMO and LUMO) in the dimers BTI4-BTI7 with maximum charge transfer integrals. 
Table S5. Charge transfer paths, transfer distances, and hole and electron charge transfer integrals in the simulated crystals of BTI series.

\begin{tabular}{|c|c|c|c|c|}
\hline compound & pathway & Distance $(\AA)$ & $\mathrm{H}_{\mathrm{h}}(\mathrm{meV})$ & $\mathrm{H}_{\mathrm{e}}(\mathrm{meV})$ \\
\hline \multirow{9}{*}{ BTI } & 1 & 9.605 & -0.62 & 0.94 \\
\hline & 2 & 10.834 & -0.41 & -0.41 \\
\hline & 3 & 4.318 & $228.29\left(258.634^{\mathrm{a}}\right)$ & $-216.83\left(-247.248^{\mathrm{a}}\right)$ \\
\hline & 4 & 4.304 & $-18.67\left(-20.955^{\mathrm{a}}\right)$ & $-88.72\left(-102.233^{a}\right)$ \\
\hline & 5 & 7.781 & -13.97 & 2.61 \\
\hline & 6 & 8.729 & 9.06 & 8.42 \\
\hline & 7 & 9.933 & -1.94 & -2.82 \\
\hline & 8,9 & 9.620 & 0.12 & 8.44 \\
\hline & 10 & 9.798 & -0.79 & -17.22 \\
\hline \multirow{6}{*}{ BTI2 } & 1,2 & 4.007 & $-192.84\left(-210.124^{\mathrm{a}}\right)$ & $-30.04\left(32.501^{a}\right)$ \\
\hline & $3,7,10,12$ & 13.556 & 0.64 & 0.59 \\
\hline & $4,5,6,9$ & 14.932 & -0.66 & 1.17 \\
\hline & 8 & 9.837 & 0.59 & -8.52 \\
\hline & 11 & 9.838 & 0.59 & -8.52 \\
\hline & 13,14 & 8.985 & -6.17 & -7.54 \\
\hline \multirow{6}{*}{ BTI3 } & 1,2 & 4.158 & $-191.72\left(-203.401^{a}\right)$ & $-98.41\left(-105.270^{\mathrm{a}}\right)$ \\
\hline & 3,4 & 15.511 & 0.00 & -0.03 \\
\hline & 5,6 & 11.252 & -1.57 & 6.10 \\
\hline & 7,8 & 11.214 & 0.37 & -0.85 \\
\hline & 9,10 & 15.279 & -0.58 & 4.35 \\
\hline & 11,12 & 16.108 & 1.17 & -1.60 \\
\hline \multirow{5}{*}{ BTI4 } & 1,2 & 4.170 & -214.93 & -98.31 \\
\hline & 3,4 & 15.951 & 0.00 & -0.02 \\
\hline & $5,8,10,14$ & 16.376 & -1.33 & 2.58 \\
\hline & $6,7,11,12$ & 15.554 & 0.40 & -0.82 \\
\hline & 9,13 & 18.144 & 0.00 & -2.22 \\
\hline \multirow{4}{*}{ BTI5 } & 1,2 & 4.138 & -223.95 & -45.39 \\
\hline & 3,4 & 10.436 & 0.42 & 3.69 \\
\hline & 5,6 & 10.436 & 0.26 & 2.50 \\
\hline & 7,8 & 9.580 & -0.47 & -7.02 \\
\hline \multirow{6}{*}{ BTI6 } & 1,2 & 4.125 & -224.10 & -75.45 \\
\hline & 3,4 & 9.721 & -0.64 & -4.78 \\
\hline & 5 & 32.256 & -47.09 & -14.00 \\
\hline & 6,7 & 9.737 & 0.46 & 8.60 \\
\hline & 8 & 32.605 & 0.22 & -0.02 \\
\hline & 9 & 32.432 & 5.40 & 2.08 \\
\hline \multirow{4}{*}{ BTI7 } & 1,2 & 4.205 & -212.60 & -75.85 \\
\hline & 3,4 & 11.324 & 0.19 & 0.67 \\
\hline & 5,6 & 9.651 & -0.75 & -6.14 \\
\hline & 7,8 & 9.665 & 0.50 & 8.80 \\
\hline \multirow{8}{*}{ BTI8 } & 1,2 & 4.136 & -222.78 & -91.76 \\
\hline & 3,4 & 9.696 & -0.72 & -6.32 \\
\hline & 5,6 & 9.590 & 0.41 & 8.36 \\
\hline & 7,8 & 43.179 & 0.08 & -0.06 \\
\hline & 9,10 & 42.843 & 1.37 & -0.44 \\
\hline & 11,12 & 42.972 & -0.10 & 0.09 \\
\hline & 13,14 & 43.267 & -0.03 & -0.02 \\
\hline & 15,16 & 42.707 & 1.32 & -1.55 \\
\hline
\end{tabular}


${ }^{\text {a }}$ Hole and electron charge transfer integrals in the simulated crystals of BTI series at LC$\operatorname{BLYP}^{*}(\omega, \alpha, \varepsilon) / 6-31 \mathrm{G}(\mathrm{d}, \mathrm{p})$ level.

Table S6. The evolution of absolute values of transfer integrals $(\mathrm{eV})$ with inter-molecular distances $\left(d_{P}\right)$, short $\left(d_{S}\right)$ and long $\left(d_{L}\right)$ axis displacements by sliding one molecule over the other in BTI3 dimers model calculated at B3LYP/6-31G(d,p) level.

\begin{tabular}{|c|c|c|c|c|c|c|c|c|}
\hline \multicolumn{3}{|c|}{$\mathrm{d}_{\mathrm{P}}$} & \multicolumn{3}{|c|}{$\mathrm{ds}_{\mathrm{s}}$} & \multicolumn{3}{|c|}{$\mathrm{d}_{\mathrm{L}}$} \\
\hline $\mathrm{r}$ & $\mathrm{t}+$ & t- & $\mathrm{r}$ & $\mathrm{t}+$ & t- & $\mathrm{r}$ & $\mathrm{t}+$ & t- \\
\hline 3.0 & 0.70 & 0.76 & 0 & $3.23 \mathrm{E}-01$ & $3.60 \mathrm{E}-01$ & 0 & $3.23 \mathrm{E}-01$ & $3.60 \mathrm{E}-01$ \\
\hline 3.1 & 0.59 & 0.64 & 0.4 & $2.45 \mathrm{E}-01$ & $3.24 \mathrm{E}-01$ & 0.2 & $2.88 \mathrm{E}-01$ & $3.36 \mathrm{E}-01$ \\
\hline 3.2 & 0.50 & 0.55 & 0.8 & $1.10 \mathrm{E}-01$ & $2.30 \mathrm{E}-01$ & 0.4 & 2.67E-01 & $2.71 \mathrm{E}-01$ \\
\hline 3.3 & 0.42 & 0.47 & 1.2 & $5.52 \mathrm{E}-02$ & 1.19E-01 & 0.6 & $2.37 \mathrm{E}-01$ & $1.86 \mathrm{E}-01$ \\
\hline 3.4 & 0.36 & 0.40 & 1.6 & $1.88 \mathrm{E}-01$ & $3.74 \mathrm{E}-02$ & 0.8 & $2.05 \mathrm{E}-01$ & $9.99 \mathrm{E}-02$ \\
\hline 3.5 & 0.30 & 0.34 & 2 & $2.42 \mathrm{E}-01$ & $8.56 \mathrm{E}-03$ & 1 & $1.73 \mathrm{E}-01$ & $2.94 \mathrm{E}-02$ \\
\hline 3.6 & 0.25 & 0.29 & 2.4 & $2.02 \mathrm{E}-01$ & $2.99 \mathrm{E}-02$ & 1.2 & $1.45 \mathrm{E}-01$ & $1.80 \mathrm{E}-02$ \\
\hline 3.7 & 0.21 & 0.25 & 2.8 & $8.77 \mathrm{E}-02$ & $7.81 \mathrm{E}-02$ & 1.4 & $1.21 \mathrm{E}-01$ & $4.05 \mathrm{E}-02$ \\
\hline 3.8 & 0.18 & 0.21 & 3.2 & $5.36 \mathrm{E}-02$ & $1.19 \mathrm{E}-01$ & 1.6 & $1.01 \mathrm{E}-01$ & $4.09 \mathrm{E}-02$ \\
\hline 3.9 & 0.15 & 0.18 & 3.6 & $1.67 \mathrm{E}-01$ & $1.24 \mathrm{E}-01$ & 1.8 & $8.62 \mathrm{E}-02$ & $2.44 \mathrm{E}-02$ \\
\hline \multirow[t]{26}{*}{4.0} & 0.13 & 0.15 & 4 & $2.13 \mathrm{E}-01$ & $7.98 \mathrm{E}-02$ & 2 & 7.44E-02 & $2.61 \mathrm{E}-03$ \\
\hline & & & 4.4 & $1.77 \mathrm{E}-01$ & $2.51 \mathrm{E}-04$ & 2.2 & $6.54 \mathrm{E}-02$ & $3.39 \mathrm{E}-02$ \\
\hline & & & 4.8 & $7.54 \mathrm{E}-02$ & $9.07 \mathrm{E}-02$ & 2.4 & $5.82 \mathrm{E}-02$ & $6.39 \mathrm{E}-02$ \\
\hline & & & 5.2 & $5.08 \mathrm{E}-02$ & $1.61 \mathrm{E}-01$ & 2.6 & $5.22 \mathrm{E}-02$ & $8.83 \mathrm{E}-02$ \\
\hline & & & 5.6 & $1.54 \mathrm{E}-01$ & $1.86 \mathrm{E}-01$ & 2.8 & 4.69E-02 & $1.05 \mathrm{E}-01$ \\
\hline & & & 6 & $1.98 \mathrm{E}-01$ & $1.59 \mathrm{E}-01$ & 3 & $4.18 \mathrm{E}-02$ & $1.12 \mathrm{E}-01$ \\
\hline & & & 6.4 & $1.71 \mathrm{E}-01$ & $6.80 \mathrm{E}-03$ & 3.2 & $3.67 \mathrm{E}-02$ & $1.10 \mathrm{E}-01$ \\
\hline & & & 6.8 & $8.50 \mathrm{E}-02$ & $2.48 \mathrm{E}-02$ & 3.4 & $3.15 \mathrm{E}-02$ & $1.00 \mathrm{E}-01$ \\
\hline & & & 7.2 & $2.43 \mathrm{E}-02$ & $2.59 \mathrm{E}-02$ & 3.6 & $2.61 \mathrm{E}-02$ & $8.46 \mathrm{E}-02$ \\
\hline & & & 7.6 & $1.17 \mathrm{E}-01$ & $3.74 \mathrm{E}-02$ & 3.8 & $2.08 \mathrm{E}-02$ & $6.55 \mathrm{E}-02$ \\
\hline & & & 8 & $1.64 \mathrm{E}-01$ & $1.13 \mathrm{E}-02$ & 4 & $1.56 \mathrm{E}-02$ & $4.51 \mathrm{E}-02$ \\
\hline & & & 8.4 & $1.53 \mathrm{E}-01$ & $3.40 \mathrm{E}-02$ & 4.2 & $1.08 \mathrm{E}-02$ & $2.49 \mathrm{E}-02$ \\
\hline & & & 8.8 & $9.14 \mathrm{E}-02$ & $7.38 \mathrm{E}-02$ & 4.4 & $6.60 \mathrm{E}-03$ & $6.52 \mathrm{E}-03$ \\
\hline & & & 9.2 & $4.91 \mathrm{E}-03$ & $8.75 \mathrm{E}-02$ & 4.6 & $3.08 \mathrm{E}-03$ & $9.37 \mathrm{E}-03$ \\
\hline & & & 9.6 & $7.54 \mathrm{E}-02$ & $6.68 \mathrm{E}-02$ & 4.8 & $3.54 \mathrm{E}-04$ & $2.23 \mathrm{E}-02$ \\
\hline & & & 10 & $1.23 \mathrm{E}-01$ & $1.90 \mathrm{E}-02$ & 5 & $1.57 \mathrm{E}-03$ & $3.24 \mathrm{E}-02$ \\
\hline & & & 10.4 & $1.25 \mathrm{E}-01$ & $3.87 \mathrm{E}-02$ & 5.2 & $2.73 \mathrm{E}-03$ & $3.97 \mathrm{E}-02$ \\
\hline & & & 10.8 & $8.44 \mathrm{E}-02$ & $8.70 \mathrm{E}-02$ & 5.4 & $3.23 \mathrm{E}-03$ & $4.46 \mathrm{E}-02$ \\
\hline & & & 11.2 & $1.95 \mathrm{E}-02$ & $1.12 \mathrm{E}-01$ & 5.6 & $3.22 \mathrm{E}-03$ & $4.76 \mathrm{E}-02$ \\
\hline & & & 11.6 & $4.50 \mathrm{E}-02$ & $1.10 \mathrm{E}-01$ & 5.8 & $2.86 \mathrm{E}-03$ & $4.90 \mathrm{E}-02$ \\
\hline & & & 12 & $8.75 \mathrm{E}-02$ & $8.57 \mathrm{E}-02$ & 6 & $2.28 \mathrm{E}-03$ & $4.91 \mathrm{E}-02$ \\
\hline & & & 12.4 & $9.64 \mathrm{E}-02$ & $5.05 \mathrm{E}-02$ & 6.2 & $1.63 \mathrm{E}-03$ & $4.80 \mathrm{E}-02$ \\
\hline & & & 12.8 & 7.29E-02 & $1.88 \mathrm{E}-02$ & 6.4 & $9.92 \mathrm{E}-04$ & $4.61 \mathrm{E}-02$ \\
\hline & & & 13.2 & $2.91 \mathrm{E}-02$ & $1.89 \mathrm{E}-03$ & 6.6 & $4.34 \mathrm{E}-04$ & $4.34 \mathrm{E}-02$ \\
\hline & & & 13.6 & $1.80 \mathrm{E}-02$ & $3.64 \mathrm{E}-03$ & 6.8 & $7.50 \mathrm{E}-06$ & $4.00 \mathrm{E}-02$ \\
\hline & & & 14 & $5.34 \mathrm{E}-02$ & $1.95 \mathrm{E}-02$ & 7 & $3.23 \mathrm{E}-04$ & $3.63 \mathrm{E}-02$ \\
\hline
\end{tabular}




\begin{tabular}{|c|c|c|c|c|c|}
\hline 14.4 & $6.80 \mathrm{E}-02$ & $3.98 \mathrm{E}-02$ & 7.2 & $5.21 \mathrm{E}-04$ & $3.23 \mathrm{E}-02$ \\
\hline 14.8 & $6.09 \mathrm{E}-02$ & $5.41 \mathrm{E}-02$ & 7.4 & $6.18 \mathrm{E}-04$ & $2.82 \mathrm{E}-02$ \\
\hline 15.2 & $3.76 \mathrm{E}-02$ & 5.61E-02 & 7.6 & $6.39 \mathrm{E}-04$ & $2.42 \mathrm{E}-02$ \\
\hline 15.6 & $8.06 \mathrm{E}-03$ & 4.54E-02 & 7.8 & $6.08 \mathrm{E}-04$ & $2.04 \mathrm{E}-02$ \\
\hline 16 & $1.80 \mathrm{E}-02$ & $2.68 \mathrm{E}-02$ & 8 & $5.44 \mathrm{E}-04$ & $1.69 \mathrm{E}-02$ \\
\hline 16.4 & $3.34 \mathrm{E}-02$ & $7.55 \mathrm{E}-03$ & 5 & $3.23 \mathrm{E}-01$ & $3.60 \mathrm{E}-01$ \\
\hline 16.8 & $3.56 \mathrm{E}-02$ & $6.20 \mathrm{E}-03$ & 5.2 & $2.88 \mathrm{E}-01$ & $3.36 \mathrm{E}-01$ \\
\hline 17.2 & $2.68 \mathrm{E}-02$ & 1.17E-02 & 5.4 & $2.67 \mathrm{E}-01$ & $2.71 \mathrm{E}-01$ \\
\hline 17.6 & $1.22 \mathrm{E}-02$ & $9.95 \mathrm{E}-03$ & 5.6 & $2.37 \mathrm{E}-01$ & $1.86 \mathrm{E}-01$ \\
\hline 18 & $2.61 \mathrm{E}-03$ & 4.05E-03 & 5.8 & $2.05 \mathrm{E}-01$ & $9.99 \mathrm{E}-02$ \\
\hline 18.4 & $1.36 \mathrm{E}-02$ & $2.44 \mathrm{E}-03$ & 6 & $1.73 \mathrm{E}-01$ & $2.94 \mathrm{E}-02$ \\
\hline 18.8 & $1.91 \mathrm{E}-02$ & 7.14E-03 & 6.2 & $1.45 \mathrm{E}-01$ & $1.80 \mathrm{E}-02$ \\
\hline 19.2 & $1.96 \mathrm{E}-02$ & $9.20 \mathrm{E}-03$ & 6.4 & $1.21 \mathrm{E}-01$ & $4.05 \mathrm{E}-02$ \\
\hline 19.6 & $1.69 \mathrm{E}-02$ & $9.01 \mathrm{E}-03$ & 6.6 & $1.01 \mathrm{E}-01$ & $4.09 \mathrm{E}-02$ \\
\hline 20 & $1.28 \mathrm{E}-02$ & $7.48 \mathrm{E}-03$ & 6.8 & $8.62 \mathrm{E}-02$ & $2.44 \mathrm{E}-02$ \\
\hline & & & 7 & $7.44 \mathrm{E}-02$ & $2.61 \mathrm{E}-03$ \\
\hline & & & 7.2 & $6.54 \mathrm{E}-02$ & $3.39 \mathrm{E}-02$ \\
\hline & & & 7.4 & $5.82 \mathrm{E}-02$ & $6.39 \mathrm{E}-02$ \\
\hline & & & 7.6 & $5.22 \mathrm{E}-02$ & 8.83E-02 \\
\hline & & & 7.8 & $4.69 \mathrm{E}-02$ & $1.05 \mathrm{E}-01$ \\
\hline & & & 8 & $4.18 \mathrm{E}-02$ & $1.12 \mathrm{E}-01$ \\
\hline & & & 6.4 & $3.67 \mathrm{E}-02$ & $1.10 \mathrm{E}-01$ \\
\hline & & & 6.6 & $3.15 \mathrm{E}-02$ & $1.00 \mathrm{E}-01$ \\
\hline & & & 6.8 & $2.61 \mathrm{E}-02$ & $8.46 \mathrm{E}-02$ \\
\hline & & & 7 & $2.08 \mathrm{E}-02$ & $6.55 \mathrm{E}-02$ \\
\hline & & & 7.2 & $1.56 \mathrm{E}-02$ & $4.51 \mathrm{E}-02$ \\
\hline & & & 7.4 & $1.08 \mathrm{E}-02$ & $2.49 \mathrm{E}-02$ \\
\hline & & & 7.6 & $6.60 \mathrm{E}-03$ & $6.52 \mathrm{E}-03$ \\
\hline & & & 7.8 & $3.08 \mathrm{E}-03$ & 9.37E-03 \\
\hline & & & 8 & $3.54 \mathrm{E}-04$ & $2.23 \mathrm{E}-02$ \\
\hline
\end{tabular}


(a)

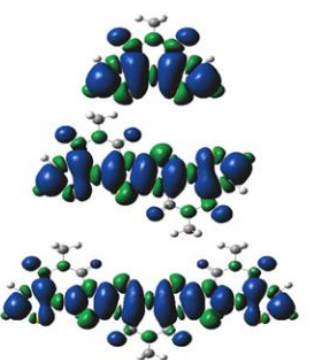

\%

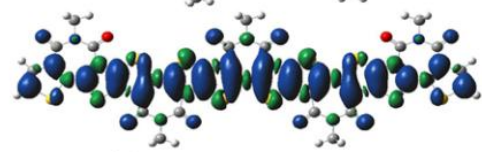

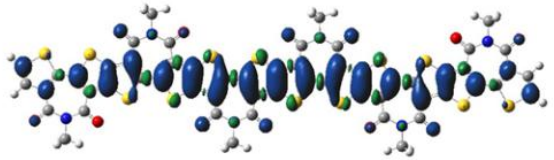

$\therefore$

: (b)
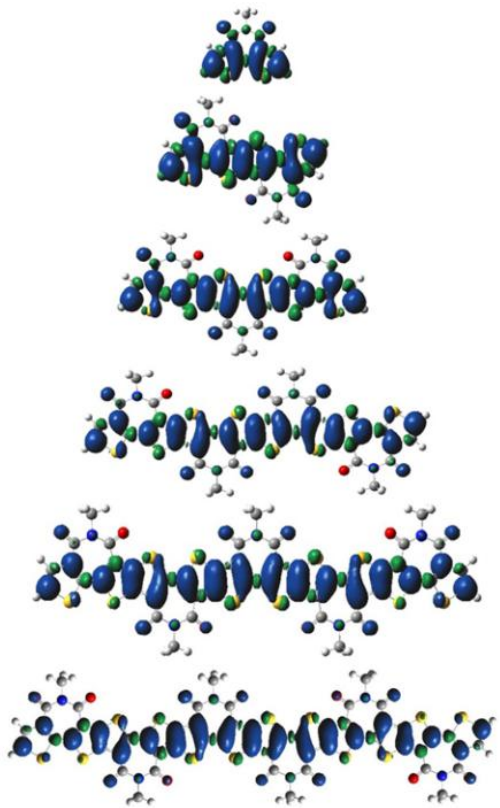

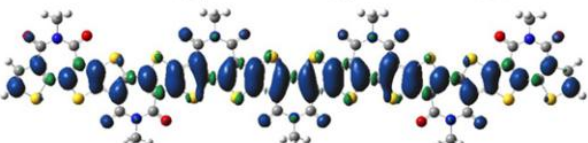

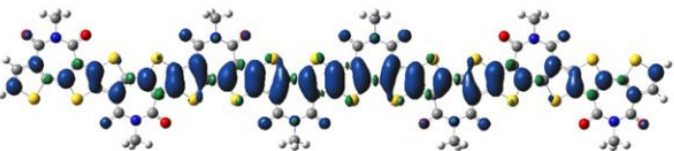

Figure S9. Spin density map in BTI-BTI8 cations calculated at the (a) LC-BLYP*/6-31G(d,p) and (b) B3LYP/6-31G(d,p) levels in a vacuum.

(a)
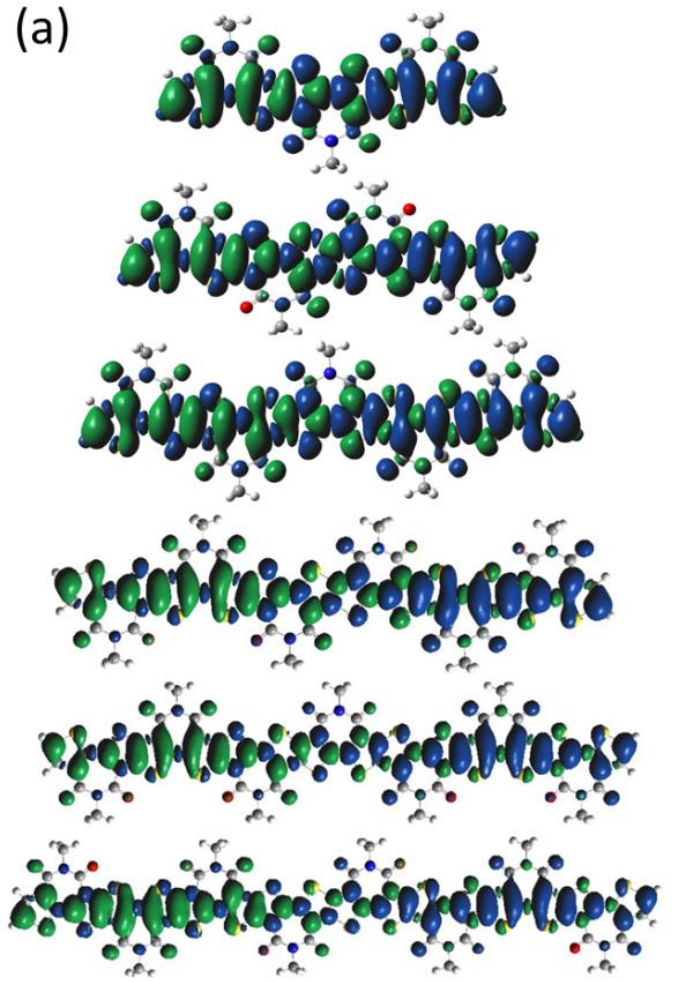

(b)
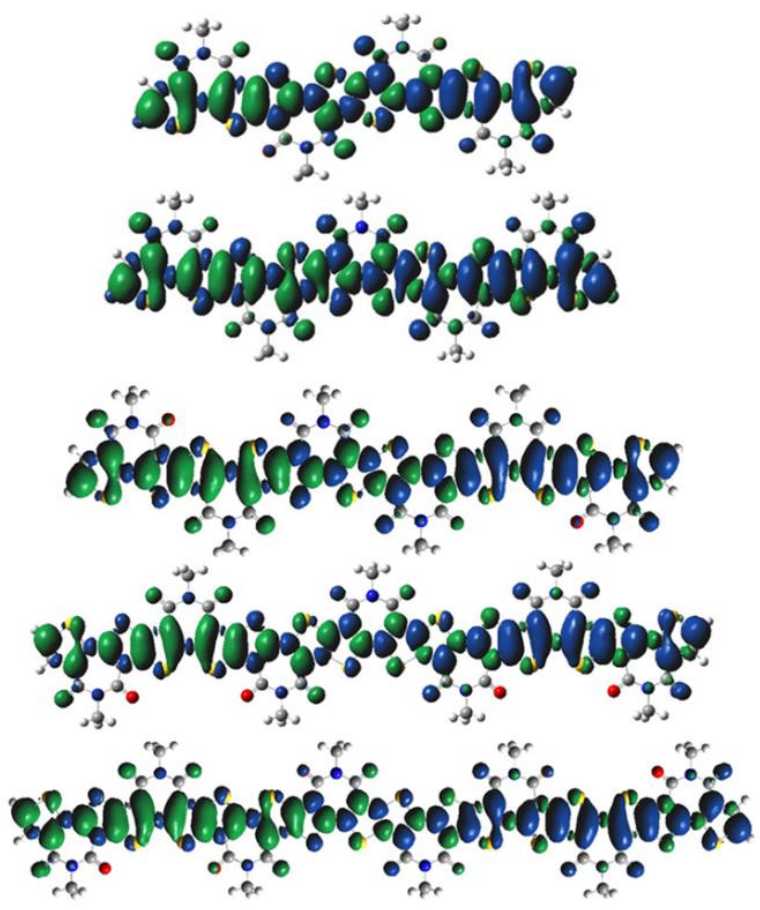

Figure S10. Spin density map of the separated polarons of (a) BTI3-BTI8 calculated at the LCBLYP*/6-31G(d,p) and (b) of BTI4-BTI8 calculated at the B3LYP/6-31G(d,p) level in a vacuum. 
(a)
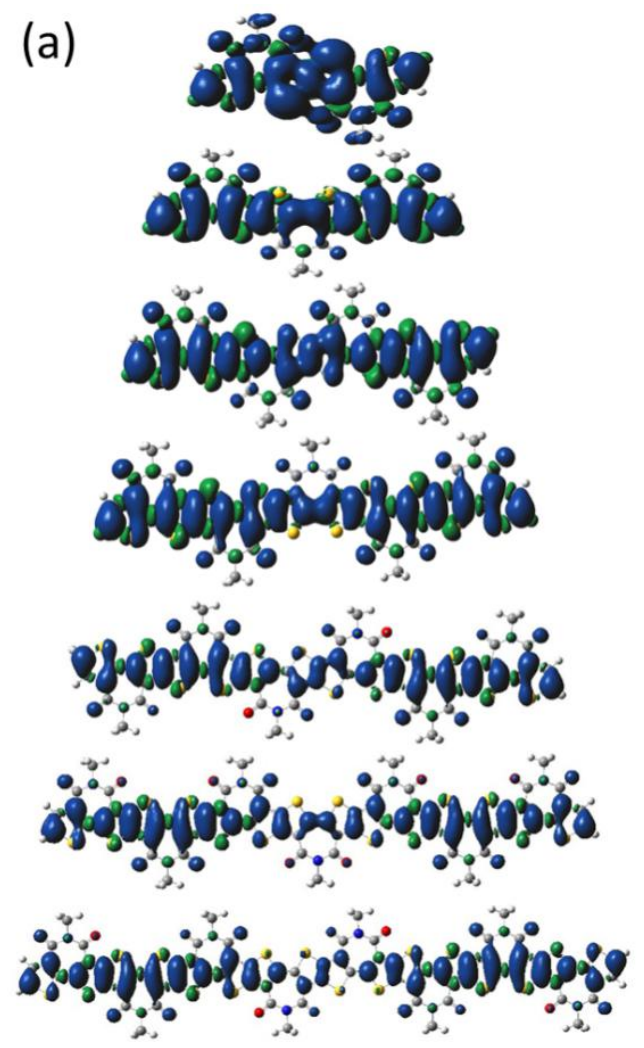

(b)
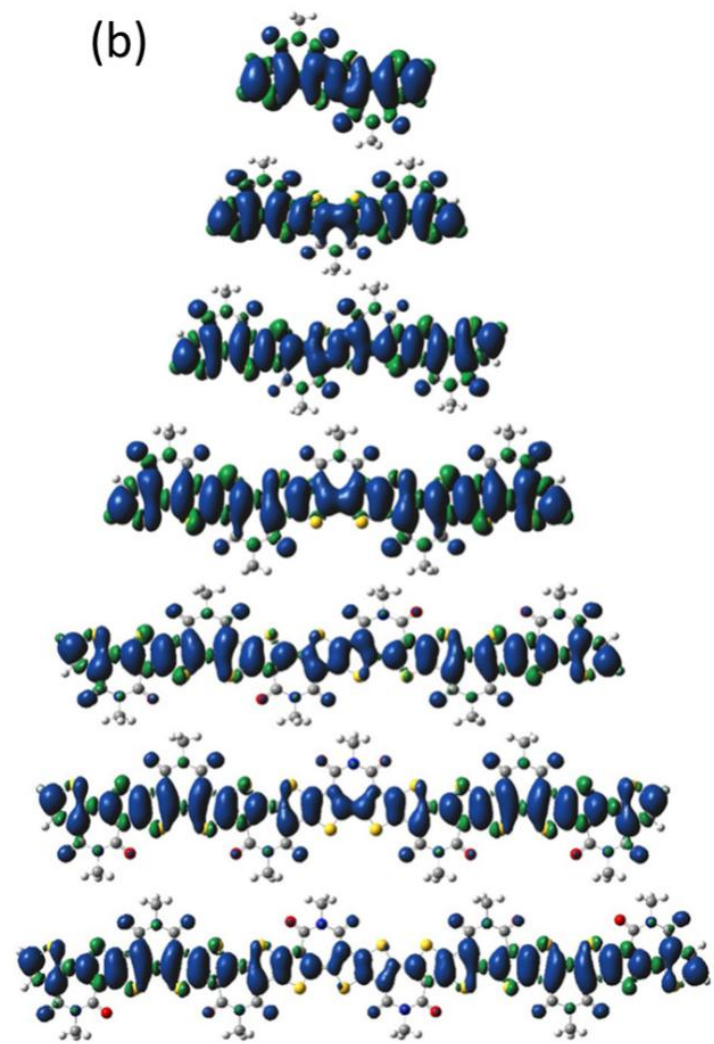

Figure S11. Spin density map in BTI2-BTI8 bipolarons calculated at the (a) LC-BLYP*/6$31 \mathrm{G}(\mathrm{d}, \mathrm{p})$ and (b) B3LYP/6-31G(d,p) levels in a vacuum.

Table S7. Comparison of the calculated spin density distributions in the BTI-BTI8 cations/ separated polarons /bipolarons calculated at the LC-BLYP*(PCM, solid)/6-31G(d,p) level.

\begin{tabular}{rcccr}
\hline & & \multicolumn{3}{c}{ Spin Density in each T/TT unit } \\
\cline { 2 - 5 } molecule & T or TT & T or TT & T or TT \\
\cline { 2 - 5 } & BTI & 0.480950 .48129 & & \\
& BTI2 & 0.243810 .459360 .24381 & & \\
& BTI3 & 0.152130 .315460 .31653 & 0.15285 & \\
& BTI4 & 0.105250 .230510 .25883 & 0.230510 .10525 & \\
& BTI5 & 0.077440 .175360 .21028 & 0.210290 .175360 .07748 & \\
& BTI6 & 0.059470 .138050 .17285 & 0.184280 .17197 & 0.137450 .05922 \\
& BTI7 & 0.046900 .110850 .14338 & 0.159480 .159490 .14339 & 0.110850 .04690 \\
Bipolarons & BTI8 & 0.040310 .095130 .12134 & 0.134960 .138950 .13497 & 0.121360 .095120 .04034 \\
\hline & BTI & -- & & \\
& BTI2 & 0.279990 .575090 .28001 & & \\
& BTI3 & 0.426480 .52498 & 0.525370 .42663 & \\
BTI4 & 0.309360 .468980 .33514 & 0.468980 .30936 & \\
& BTI5 & 0.234470 .404610 .30123 & 0.301140 .404680 .23455 & \\
& BTI6 & 0.183750 .345480 .29056 & 0.232140 .29017 & 0.345640 .18392 \\
BTI7 & 0.148040 .295180 .27559 & 0.213340 .213340 .27559 & 0.295180 .14805 \\
& BTI8 & 0.121750 .253830 .25631 & 0.208510 .177710 .20852 & 0.256320 .253740 .12174 \\
\hline
\end{tabular}


(a)

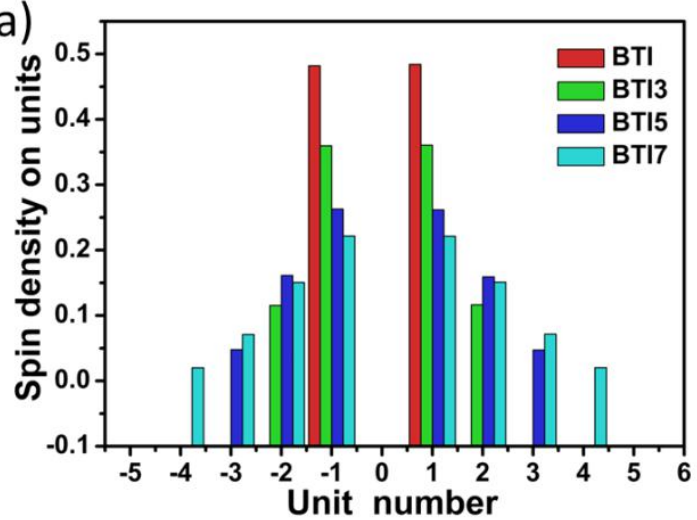

(c)

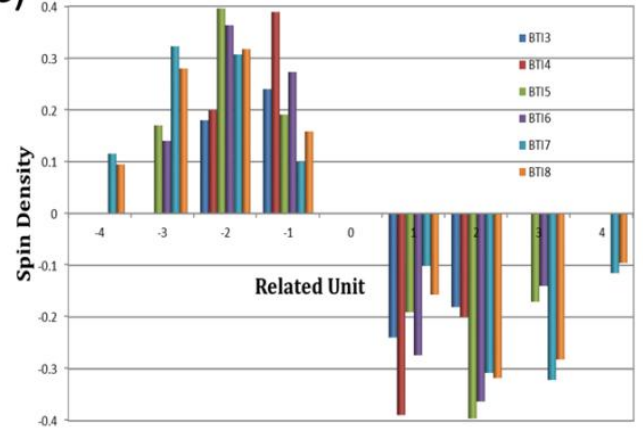

(b)

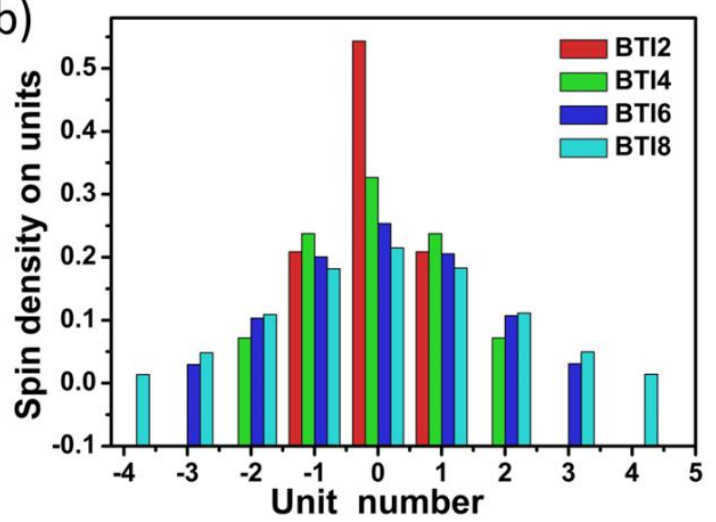

(d)

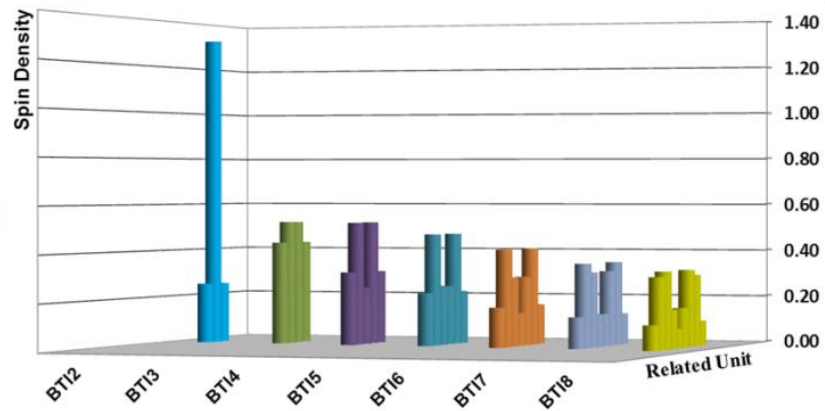

Figure S12. $(a, b)$ Distribution of spin density calculated at the LC-BLYP*/6-31G(d,p) level on each T unit in the BTI-BTI8 cations.(c) in the BTI3-BT8 separated polarons.(d) in the BTI2-BTI8 bipolarons in a vacuum.

Table S8. Comparison of the calculated spin density distributions in the BTI-BTI8 cations/ separated polarons /bipolarons calculated at the LC-BLYP*/6-31G(d,p) level.

\begin{tabular}{|c|c|c|c|c|}
\hline & \multirow{2}{*}{ molecule } & \multicolumn{3}{|c|}{ Spin Density in each T/TT unit } \\
\hline & & $\mathrm{T}$ or TT & $\mathrm{T}$ or TT & $\mathrm{T}$ or TT \\
\hline \multirow{8}{*}{ cations } & BTI & 0.482080 .48425 & & \\
\hline & BTI2 & 0.208640 .543190 .20864 & & \\
\hline & BTI3 & 0.115350 .359650 .36054 & 0.11640 & \\
\hline & BTI4 & 0.071890 .237480 .32660 & 0.237480 .07189 & \\
\hline & BTI5 & 0.047900 .161300 .26285 & 0.261740 .159290 .04730 & \\
\hline & BTI6 & 0.029580 .103290 .20069 & 0.253580 .205550 .10721 & 0.03076 \\
\hline & BTI7 & 0.020150 .070980 .15068 & 0.221590 .221340 .15102 & 0.071620 .02033 \\
\hline & BTI8 & 0.013670 .048290 .10905 & 0.181660 .214930 .18299 & 0.111240 .049710 .01408 \\
\hline \multirow{8}{*}{$\begin{array}{l}\text { Separated } \\
\text { Polarons }\end{array}$} & BTI & - & & \\
\hline & BTI2 & & & \\
\hline & BTI3 & $0.180180 .24031-0.23956$ & -0.18080 & \\
\hline & BTI4 & 0.199450 .38897 1.00E-06 & $-0.38897-0.19945$ & \\
\hline & BTI5 & 0.170100 .395840 .19104 & $-0.19079-0.39597$ & -0.17040 \\
\hline & BTI6 & 0.140310 .363360 .27326 & $-0.00015-0.27370$ & $-0.36301-0.13986$ \\
\hline & BTI7 & $\begin{array}{c}0.115610 .322730 .30711 \\
-0.11502\end{array}$ & $0.09966-0.10106$ & $-0.30756-0.32154$ \\
\hline & BTI8 & $\begin{array}{c}0.095030 .280070 .31740 \\
-0.09568\end{array}$ & $0.158480 .00077-0.15667$ & $-0.31752-0.28179$ \\
\hline
\end{tabular}




\begin{tabular}{ccccc}
\hline \multicolumn{6}{c}{ BTI } & \multicolumn{1}{c}{ - } & & \\
& BTI2 & 0.253971 .311780 .25402 & & \\
& BTI3 & 0.435560 .52345 & 0.523760 .43510 & \\
Bipolarons & BTI4 & 0.309730 .522120 .24108 & 0.522120 .30973 & \\
& BTI5 & 0.223650 .473410 .24928 & 0.248670 .473690 .22418 & 0.16815 \\
& BTI6 & 0.168910 .412280 .29128 & 0.138580 .292150 .41162 & 0.354330 .13173 \\
& BTI7 & 0.131330 .353540 .31646 & 0.136030 .135470 .31622 & 0.324130 .302550 .10518 \\
\hline
\end{tabular}

(a)

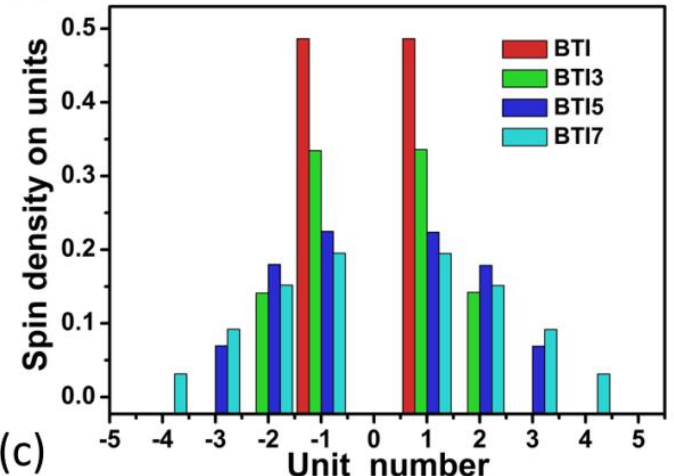

(c)

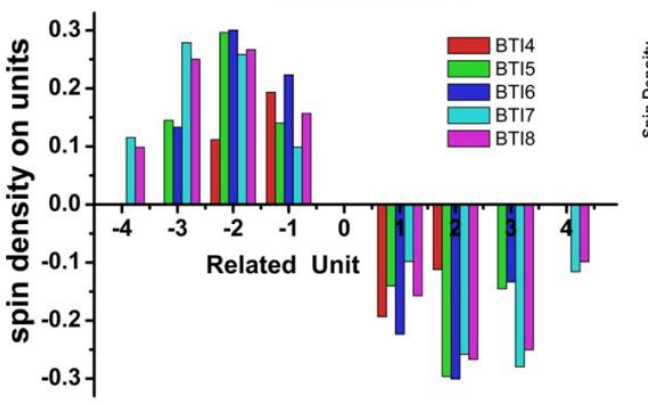

(b)

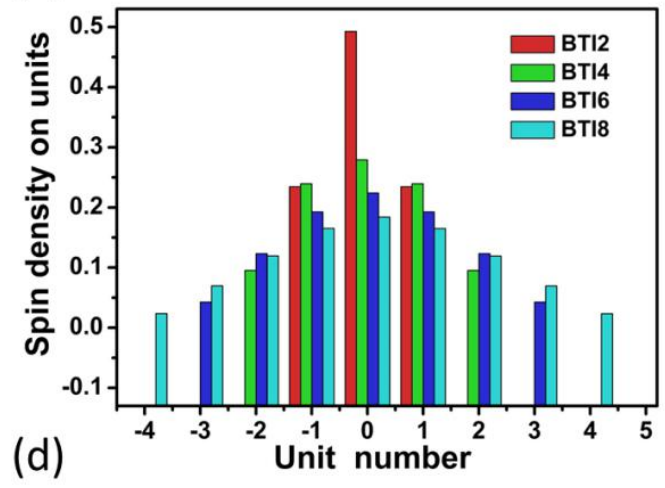

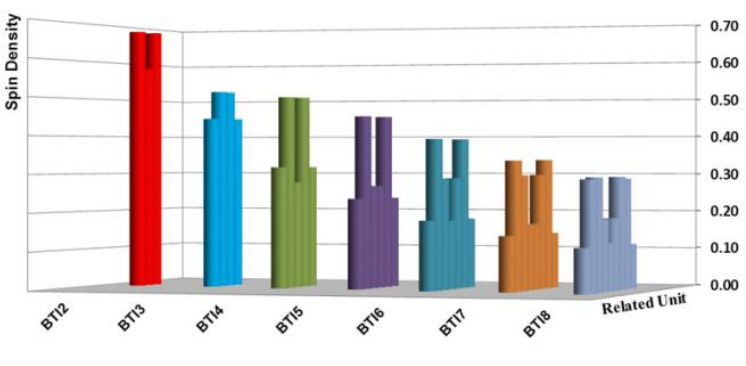

Figure S13. (a,b) Distribution of spin density calculated at the B3LYP/6-31G(d,p) level on each $T$ units in the BTI-BTI8 cations.(c) in the BTI4-BT8 separated polarons.(d) in the BTI-BTI8 Bipolarons in a vacuum.

Table S9. Comparison of the calculated spin density distributions in the BTI-BTI5 cations/ separated polarons /bipolarons calculated at the B3LYP/6-31G(d,p) level in a vacuum.

\begin{tabular}{ccccc}
\hline \multirow{4}{*}{ molecule } & \multicolumn{3}{c}{ Spin Density in each T/TT unit } \\
\cline { 3 - 5 } & BTI & 0.486360 .48642 & T or TT & T or TT \\
\hline \multirow{5}{*}{ cations } & BTI2 & 0.234460 .49263 & & \\
& BTI3 & 0.141030 .33447 & 0.335970 .14205 & \\
& BTI4 & 0.095240 .239500 .27907 & 0.239500 .09524 & \\
& BTI5 & 0.069480 .179920 .22479 & 0.223750 .178610 .06901 & \\
& BTI6 & 0.042680 .123140 .19255 & 0.223930 .19253 & 0.123140 .04267 \\
& BTI7 & 0.031340 .091880 .15183 & 0.195130 .194840 .15143 & 0.091710 .03129 \\
& BTI8 & 0.023510 .069720 .11937 & 0.165200 .183940 .16500 & 0.119080 .069480 .02339
\end{tabular}




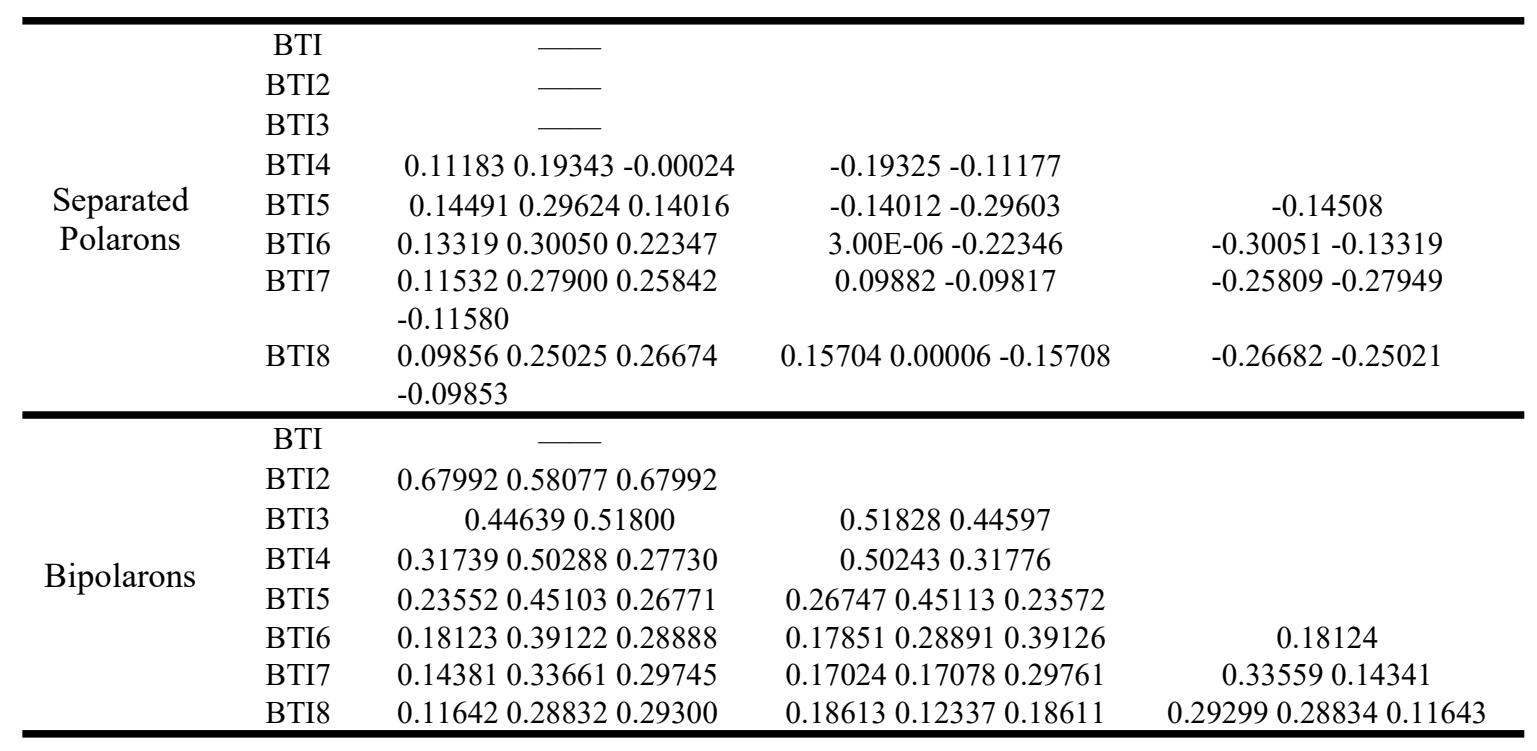

Complete list of authors for Ref. 36.

(36) Gaussian 16, Revision B.01, Frisch, M. J.; Trucks, G. W.; Schlegel, H. B.; Scuseria, G. E.; Robb, M. A.; Cheeseman, J. R.; Scalmani, G.; Barone, V.; Petersson, G. A.; Nakatsuji, H.; Li, X.; Caricato, M.; Marenich, A. V.; Bloino, J.; Janesko, B. G.; Gomperts, R.; Mennucci, B.; Hratchian, H. P.; Ortiz, J. V.; Izmaylov, A. F.; Sonnenberg, J. L.; Williams-Young, D.; Ding, F.; Lipparini, F.; Egidi, F.; Goings, J.; Peng, B.; Petrone, A.; Henderson, T.; Ranasinghe, D.; Zakrzewski, V. G.; Gao, J.; Rega, N.; Zheng, G.; Liang, W.; Hada, M.; Ehara, M.; Toyota, K.; Fukuda, R.; Hasegawa, J.; Ishida, M.; Nakajima, T.; Honda, Y.; Kitao, O.; Nakai, H.; Vreven, T.; Throssell, K.; Montgomery, J. A., Jr.; Peralta, J. E.; Ogliaro, F.; Bearpark, M. J.; Heyd, J. J.; Brothers, E. N.; Kudin, K. N.; Staroverov, V. N.; Keith, T. A.; Kobayashi, R.; Normand, J.; Raghavachari, K.; Rendell, A. P.; Burant, J. C.; Iyengar, S. S.; Tomasi, J.; Cossi, M.; Millam, J. M.; Klene, M.; Adamo, C.; Cammi, R.; Ochterski, J. W.; Martin, R. L.; Morokuma, K.; Farkas, O.; Foresman, J. B.; Fox, D. J. Gaussian, Inc., Wallingford CT, 2016. 